\title{
The Diverse Roles of Arrestin Scaffolds in G Protein-Coupled Receptor Signaling
}

Yuri K. Peterson and Louis M. Luttrell

Department of Drug Discovery and Biomedical Sciences, College of Pharmacy (Y.K.P.), and Departments of Medicine and Biochemistry and Molecular Biology (L.M.L.), Medical University of South Carolina, Charleston, South Carolina; and Ralph H. Johnson Veterans Affairs Medical Center, Charleston, South Carolina (L.M.L.)

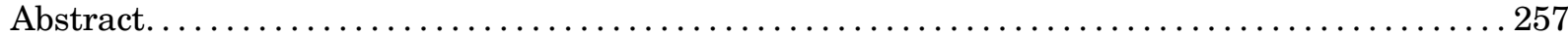

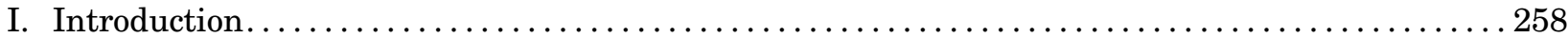

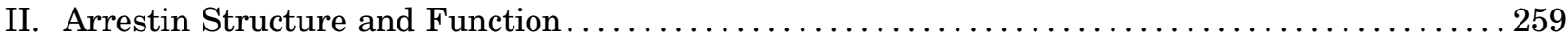

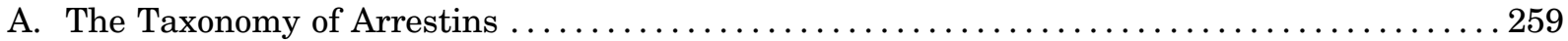

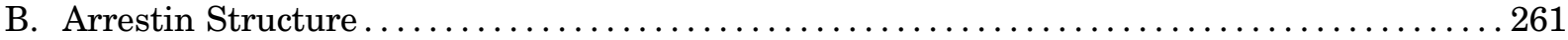

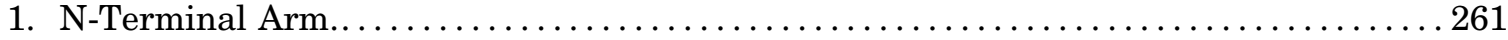

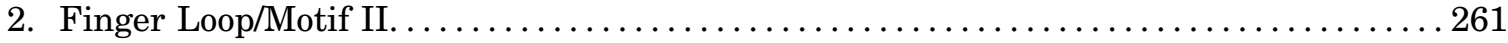

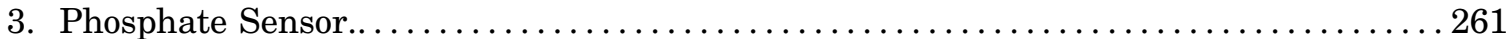

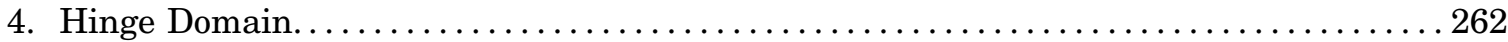

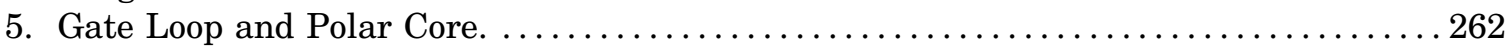

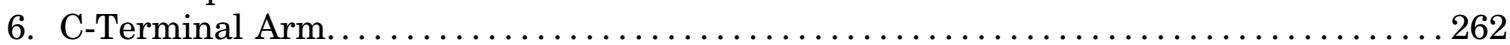

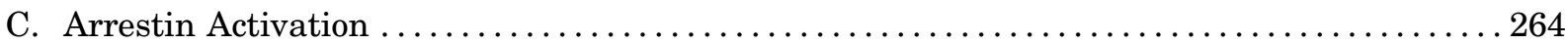

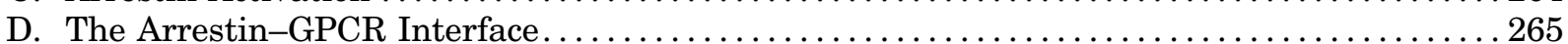

III. Visual $/ \beta$-Arrestins as Scaffolds. . . . . . . . . . . . . . . . . . . . . . . . . . . . . . . . . . 267

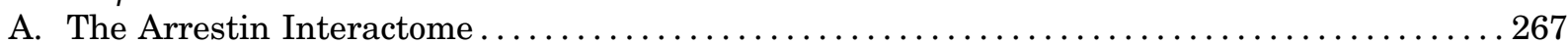

1. Clathrin-Dependent Endocytic Machinery. ................................267

2. Tubulin and Microtubules. ...................................... 267

3. Phosphoinositides. ................................................ 267

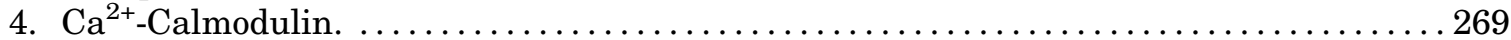

5. Src Family Nonreceptor Tyrosine Kinases............................ 269

6. Mitogen-Activated Protein Kinases................................... 269

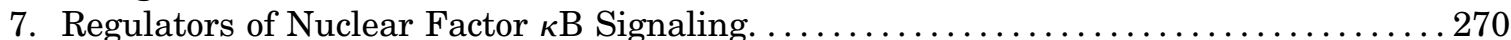

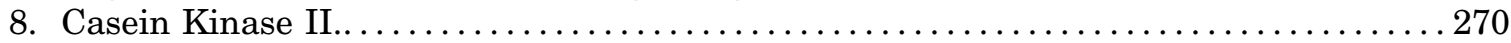

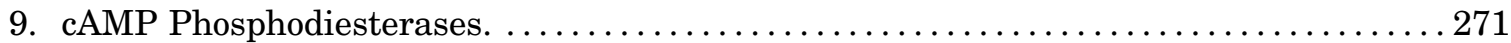

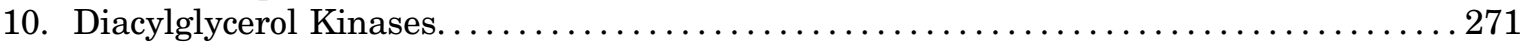

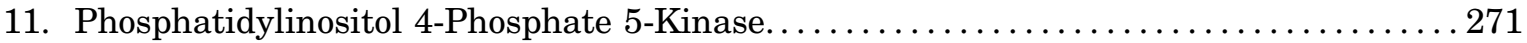

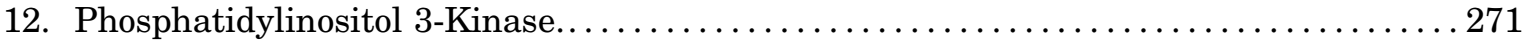

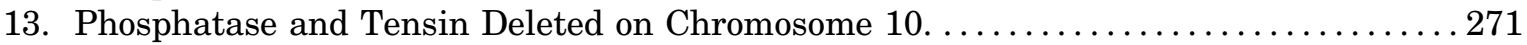

14. Protein Phosphatase 2A-Akt-Glycogen Synthase Kinase $3 \beta \ldots \ldots \ldots \ldots \ldots \ldots \ldots \ldots 271$

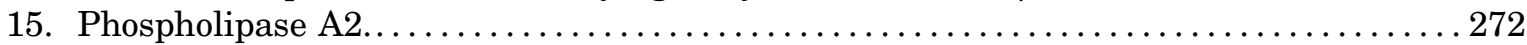

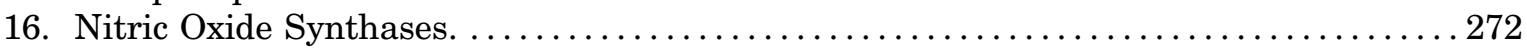

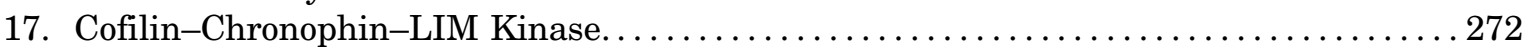

18. Phosphotyrosine Phosphatases..................................... 272

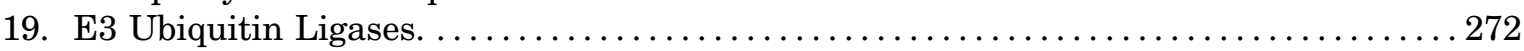

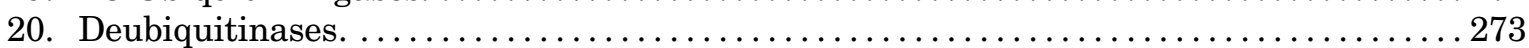

This work was supported by National Institutes of Health [Grants R01 DK055524 (to L.M.L.) and R01 GM095497 (to L.M.L.)], Department of Veterans Affairs Merit Review Grant I01 BX003188 (to L.M.L.), and the Research Service of the Charleston, South Carolina, Veterans Affairs Medical Center. The contents of this article do not represent the views of the Department of Veterans Affairs or the United States Government.

Address correspondence to: Dr. Louis M. Luttrell, Division of Endocrinology, Diabetes, and Medical Genetics, Medical University of South Carolina, 96 Jonathan Lucas Street, MSC 624, Charleston, SC 29426. E-mail: luttrell@musc.edu https://doi.org/10.1124/pr.116.013367. 


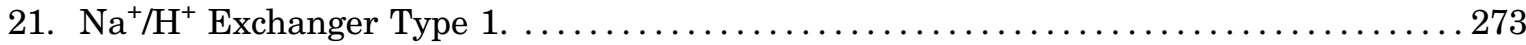

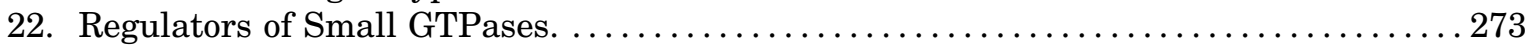

23. N-Ethylmaleimide-Sensitive Fusion Protein........................... 274

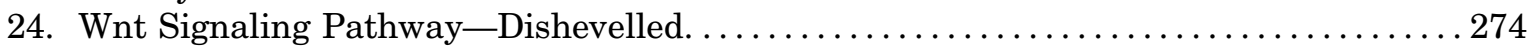

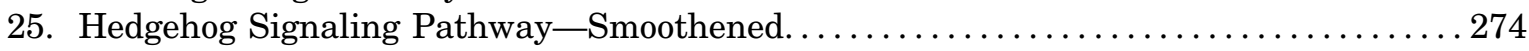

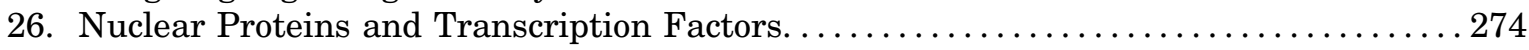

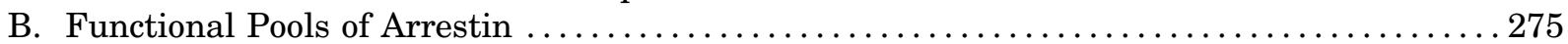

1. Cytosolic Arrestin. ............................................. 275

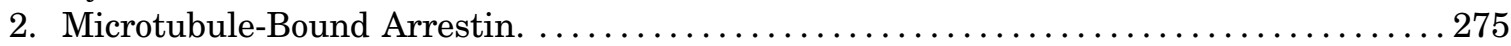

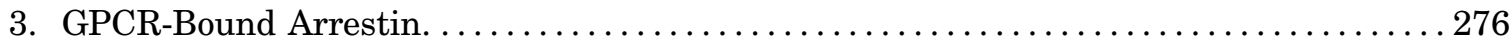

4. Nuclear Arrestin. ............................................. 277

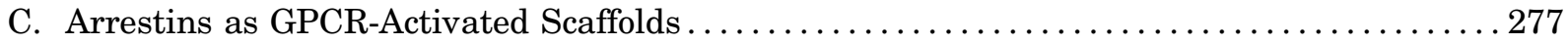

1. Negative Regulation of Heterotrimeric G Protein Signaling. ................... 277

2. Cell Proliferation. ............................................... 279

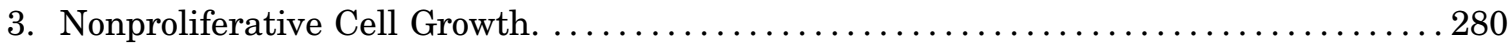

4. Cell Survival and Apoptosis......................................... 280

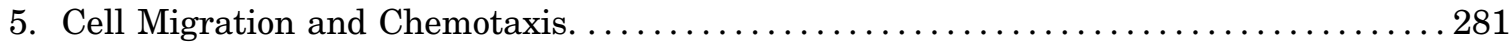

6. Modulation of the Immune Response............................... 282

7. Developmental Regulation. ...................................... 282

8. Central Nervous System Function, Learning, and Behavior.................. 283

D. Silent Scaffolds and Tonic Effects on Pathway Activity ........................ 283

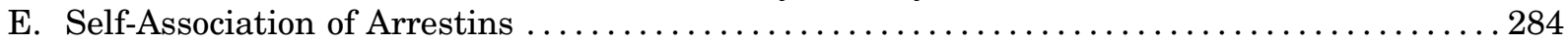

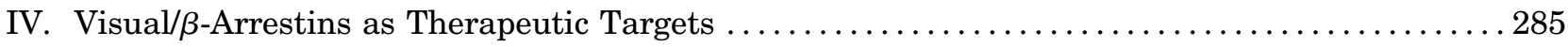

A. Orthosteric and Allosteric Modulation of GPCR Signaling $\ldots \ldots \ldots \ldots \ldots \ldots \ldots \ldots \ldots \ldots 285$

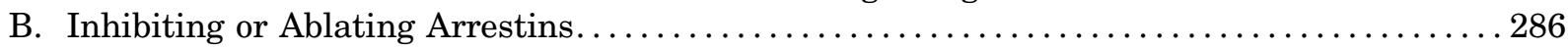

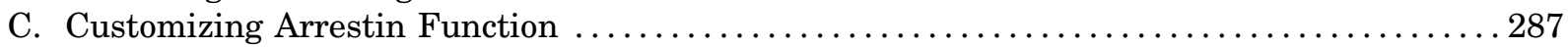

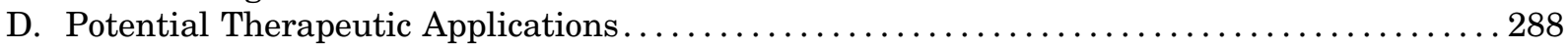

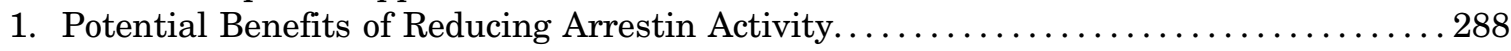

2. Benefits of Promoting Arrestin Activity. . . . . . . . . . . . . . . . . . . . . . . . . 289

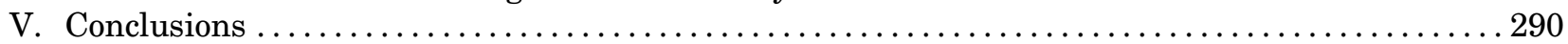

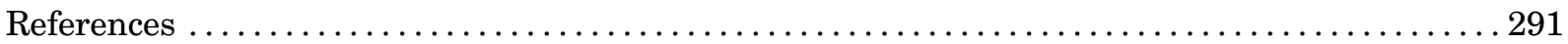

\begin{abstract}
The visual/ $\beta$-arrestins, a small family of proteins originally described for their role in the desensitization and intracellular trafficking of $G$ proteincoupled receptors (GPCRs), have emerged as key regulators of multiple signaling pathways. Evolutionarily related to a larger group of regulatory scaffolds that share a common arrestin fold, the visual $/ \beta$-arrestins acquired the capacity to detect and bind activated GPCRs on the plasma membrane, which enables them to control GPCR
\end{abstract}

desensitization, internalization, and intracellular trafficking. By acting as scaffolds that bind key pathway intermediates, visual $/ \beta$-arrestins both influence the tonic level of pathway activity in cells and, in some cases, serve as ligand-regulated scaffolds for GPCR-mediated signaling. Growing evidence supports the physiologic and pathophysiologic roles of arrestins and underscores their potential as therapeutic targets. Circumventing arrestin-dependent GPCR desensitization may alleviate

\footnotetext{
ABBREVIATIONS: AKT, protein kinase B; AP-2, adapter protein-2; ARRDC, arrestin domain-containing protein; ASK, apoptosis signal regulating kinase; BRET, bioluminescence resonance energy transfer; CML, chronic myelogenous leukemia; cPLA2, cytosolic phospholipase A2; CXCR, CXC chemokine receptor; Dsh, dishevelled; EGF, epidermal growth factor; ERK, extracellular signal-regulated kinase; FlAsH, fluorescent aresincal hairpin; FRET, fluorescence resonance energy transfer; Fz, Frizzled; GAP, GTPase-activating protein; GDS, guanine nucleotide dissociation stimulator; GEF, guanine nucleotide exchange factor; GPCR, G protein-coupled receptor; GRK, G protein-coupled receptor kinase; GSK3 $\beta$, glycogen synthase kinase 3 $\beta$; ICL, intracellular loop; IL, interleukin; iNOS, inducible nitric oxide synthase; IP6, inositol hexakisphosphate; JNK, c-Jun N-terminal kinase; LEF, lymphoid enhancer factor; LH, luteinizing hormone; LPA, lysophosphatidic acid; MAP, mitogen-activated protein; MAPK, MAP kinase; MEK, MAPK/ERK kinase; mGluR, metabotropic glutamate receptor; MKK, MAPK kinase; MOR, $\mu$ opioid receptor; NES, nuclear export sequence; NFкB, nuclear factor $\kappa B$; NHE, Na+/H+ exchanger; NLS, nuclear localization sequence; NSF, N-ethylmaleimide-sensitive fusion protein; PAR, protease-activated receptor; PcG, polycomb group; PDB, Protein Data Bank; PDE, phosphodiesterase; PDK1, 3-phosphoinositide dependent protein kinase-1; PDZ, postsynaptic density protein of 95 kDa, disc large, zona occludens-1; PI3K, phosphatidylinositol 3-kinase; PIP2, phosphatidylinositol 4,5-bisphosphate; PKA, protein kinase A; PKC, protein kinase C; PLC, phospholipase C; PP, protein phosphatase; PPAR, peroxisome proliferator-activated receptor; PTEN, phosphatase and tensin deleted on chromosome 10; PTH, parathyroid hormone; RXR, retinoic acid receptor; SH, Src homology; Shh, Sonic hedgehog; SHP, Src homology region 2 domain-containing phosphatase; SII, [Sar1,Ile4,Ile8]-angiotensin II; SNAP, soluble NSF attachment protein; SNARE, SNAP receptor; SpoOM, sporulation stage 0, protein M; STAT, signal transducer and activator of transcription; TLR, Toll-like receptor; TM, transmembrane domain; TNF- $\alpha$, tumor necrosis factor- $\alpha$; TP, thromboxane prostanoid; TRAF, TNF receptor-associated factor; TXNIP, thioredoxin-interacting protein; USP33, ubiquitin-specific protease 33; VPS, vacuollar protein sort.
} 
the problem of tachyphylaxis to drugs that target GPCRs, and find application in the management of chronic pain, asthma, and psychiatric illness. As signaling scaffolds, arrestins are also central regulators of pathways controlling cell growth, migration, and survival, suggesting that manipulating their scaffolding functions may be beneficial in inflammatory diseases, fibrosis, and cancer. In this review we examine the structure-function relationships that enable arrestins to perform their diverse roles, addressing arrestin structure at the molecular level, the relationship between arrestin conformation and function, and sites of interaction between arrestins, GPCRs, and nonreceptor-binding partners. We conclude with a discussion of arrestins as therapeutic targets and the settings in which manipulating arrestin function might be of clinical benefit.

\section{Introduction}

The complexity of coordinated cell signaling has necessitated the evolution of scaffold proteins whose role is to control the activity of cellular processes driven by receptors, enzymes, and channels. Scaffolds, proteins or protein domains that themselves lack intrinsic catalytic activity, perform three basic functions: to increase the efficiency of information transfer between successive enzymes in a signaling cascade; to enhance fidelity by dampening crosstalk between parallel cascades; and to target effectors to specific subcellular locations. The true arrestins, consisting of two retinal isoforms, visual arrestin (arrestin1) and cone arrestin (arrestin4), and two nonvisual arrestins, $\beta$-arrestin1 (arrestin2) and $\beta$-arrestin2 (arrestin3), belong to a superfamily of structurally and functionally related scaffolding proteins that trace their origins to prokaryotes and occur in all eukaryotes except plants (Ferguson, 2001; Alvarez, 2008). Depending on the type of cell and its metabolic state, arrestin family proteins can be found distributed diffusely in the cytosol, bound to the cytoskeleton, concentrated at the centrosome, coating internalizing endosomes, and inside the nucleus. These many pools of arrestin are integral to the control of cell metabolism, division, motility, and crosstalk and are adapted to provide diverse but highly specific signal integration.

What distinguishes the visual $/ \beta$-arrestins from other arrestin-like proteins is their capacity to interact with activated heptahelical $G$ protein-coupled receptors (GPCRs). Upon ligand binding, G protein-coupled receptor kinases (GRKs) phosphorylate agonist-occupied receptors on serine or threonine residues within the $\mathrm{C}$ terminus or third intracellular loop, creating highaffinity arrestin binding sites. There are seven known GRKs, of which GRK1 and 7, like visual and cone arrestin, are confined to visual sensory tissue, whereas GRK2, 3, 5, and 6 , along with $\beta$-arrestin 1 and 2 , are widely expressed (Stoffel et al., 1997). Arrestin binding stabilizes a high agonist affinity state of the receptor, similar to the complex existing between agonist, receptor, and heterotrimeric $\mathrm{G}$ protein in the absence of GTP (De Lean et al., 1980; Gurevich et al., 1997). The traditional view is that once bound to arrestin, GRKphosphorylated GPCRs on the plasma membrane are precluded from $\mathrm{G}$ protein coupling, leading to homologous desensitization, the process whereby $\mathrm{G}$ protein signaling by agonist-occupied receptors is selectively dampened (Ferguson, 2001). $\beta$-Arrestin 1 and 2 further attenuate $G$ protein signaling by linking receptors to the clathrin-dependent endocytic machinery. The $\beta$-arrestin $\mathrm{C}$ terminus directly binds clathrin heavy chain and the $\beta 2$ adaptin subunit of the adapter protein2 (AP-2) complex (Goodman et al., 1996; Krupnick et al., 1997; Laporte et al., 1999, 2000), causing $\beta$-arrestinbound receptors to cluster in clathrin-coated pits. This $\beta$-arrestin-dependent endocytosis, or sequestration, removes receptors from the cell surface, rendering it less responsive to subsequent stimuli. Once inside, the stability of the GPCR- $\beta$-arrestin complex determines whether receptors resensitize and recycle to the cell surface or are degraded, with receptors that form transient receptor- $\beta$-arrestin complexes undergoing rapid resensitization and recycling back to the plasma membrane, whereas receptors that form more stable complexes either recycle slowly or are targeted for degradation (Oakley et al., 2000, 2001).

The capacity to recognize and bind activated GPCRs is what places the scaffolding functions of visual/ $\beta$-arrestins under the control of environmental cues delivered in the form of extracellular hormones. As a result, they can play both silent scaffolding roles, binding and sequestering key signaling pathway intermediates away from potential regulators, and stimulusdependent scaffolding roles in the positive and negative regulation of GPCR signaling (Luttrell and GestyPalmer, 2010; Breitman et al., 2012; Lin and Defea, 2013). Besides clathrin and AP-2, the list of proteins that have been reported to bind arrestins includes Src family tyrosine kinases (Luttrell et al., 1999; Barlic et al., 2000; DeFea et al., 2000a), components of the extracellular signal-regulated kinase 1 and 2 (ERK1/2) and c-Jun N-terminal kinase (JNK)3 mitogenactivated protein (MAP) kinase cascades (DeFea et al., 2000b; McDonald et al., 2000; Luttrell et al., 2001), the Ser/Thr protein phosphatase (PP)2A (Beaulieu et al., 2005), E3 ubiquitin ligases and deubiquitinases (Shenoy et al., 2001, 2008, 2009), second-messenger degrading cAMP phosphodiesterases (PDE) (Perry et al., 2002) and diacylglycerol kinase (Nelson et al., $2007)$, elements of the nuclear factor $\kappa \mathrm{B}(\mathrm{NF} \kappa \mathrm{B})$ signaling pathway (Witherow et al., 2004), and regulators of small GTPase activity (Claing et al., 2001; Bhattacharya et al., 2002). It is the interaction with this diverse set of partners that positions arrestins as 
critical regulators of GPCR signal transduction and permits them to integrate GPCR-mediated signals with other inputs. In this review, we examine the structurefunction relationships that enable arrestins to perform their diverse roles, addressing arrestin structure at the molecular level, the relationship between arrestin conformation and function, and sites of interaction between arrestins, GPCRs, and nonreceptor-binding partners. We conclude with a discussion of arrestins as therapeutic targets, and the settings in which manipulating arrestin function might be of clinical benefit.

\section{Arrestin Structure and Function}

\section{A. The Taxonomy of Arrestins}

In mammals, the extended family of arrestin-like proteins is composed of at least 12 proteins that employ a conserved protein fold to coordinate the temporal and spatial aspects of multiple processes, particularly those related to endosome trafficking, vesicle sorting, and signaling (de Mendoza et al., 2014). Various synonyms for visual/b-arrestins appear in the literature: Visual arrestin (Gene Symbol: SAG) is also called arrestin-1, $\mathrm{S}$-antigen, $48 \mathrm{kDa}$ protein, or rod arrestin; $\beta$-arrestin1 (Gene Symbol: ARRB1) is also called $\beta$-arrestin or arrestin-2; $\beta$-arrestin2 (Gene Symbol: ARRB2) is also called arrestin- 3 and hTHY-ARRX; and cone arrestin (Gene Symbol: ARR3) is also called arrestin-4, arrestin$\mathrm{C}$, or $\mathrm{X}$-arrestin. The tree that gave rise to arrestins traces its roots to prokaryotes (Alvarez, 2008). The progenitor arrestin-like proteins in Archaea are known as the sporulation stage 0 , protein $\mathrm{M}(\mathrm{SpoOM})$ family, a group of proteins that function as integrators of lipid/ nutrient internalization and whose loss or overexpression blocks sporulation (Han et al., 1998; Birko et al., 2009). Eukaryotic arrestins appear to arise from the SpoOM lineage, later diverging into two major families: the $\alpha$-arrestins, consisting of the arrestin domaincontaining proteins (ARRDCs), thioredoxin-interacting protein (TXNIP), and vacuolar protein sort (VPS) 26, which coordinate enzyme activity largely for membrane trafficking; and the visual $/ \beta$-arrestins, which possess the ability to interact with GPCRs (Aubry and Klein, 2013).

The eukaryotic $\alpha$-arrestins appear to have evolved from a single progenitor. The function of the five mammalian ARRDCs is not well understood. ARRDC1-4, along with TXNIP, are known to interact with several E3 ubiquitin ligases and promote the ubiquitination of associated cargo proteins. For example, ARRDC3 binds activated $\beta-4$ integrin and controls its ubiquitination, endocytosis, and degradation (Becuwe et al., 2012). ARRDC3 has been reported to recruit the E3 ligase NEDD4 to regulate ubiquitination and endocytosis of the $\beta 2$-adrenergic receptor, although other authors attribute this function to the visual $/ \beta$-arrestin, $\beta$-arrestin 2
(Nabhan et al., 2010; Han et al., 2013). ARRDC1-4 and TXNIP also interact with subunits of the endosomal sorting complexes required for transport machinery. Consistent with a general role in vesicle trafficking, ARRDC4 and TXNIP inhibit glucose uptake, suggesting a role in endosomal trafficking of glucose transporters (Patwari et al., 2009). TNXIP also controls cellular redox state by binding and inhibiting thioredoxin (World et al., 2011; Spindel et al., 2014; Yoshihara et al., 2014). The VPS proteins are critical components of the retromer complex that sorts and traffics endosomes to the Golgi to recycle cell surface receptors and other proteins (Haft et al., 2000; Seaman, 2004). One of the three VPS proteins, VPS26, is an arrestin-fold protein (Shi et al., 2006). VPS26 engages postsynaptic density protein of $95 \mathrm{kDa}$, disc large, zona occludens-1 (PDZ) domain-containing proteins like the sorting nexin, SNX27, to regulate intracellular protein trafficking. The VPS26-SNX27 interaction promotes cooperative binding between the SNX27 PDZ domain and PDZ-binding motifs on cargo proteins (Gallon et al., 2014).

The oldest visual $/ \beta$-arrestin in our current genetic record appears in cnidarians. Cnidarians developed ocelli in the absence of a nervous system, most likely to sense the diurnal cycle for feeding and simple navigation (Nordstrom et al., 2003). GPCRs and visual/ $\beta$-arrestins evolved very early and in tandem in the earliest eukaryotes (de Mendoza et al., 2014). Given that $\alpha$-arrestins and visual $/ \beta$-arrestins share roles in vesicle trafficking, one might speculate that the shared functions originated first, with the visual $/ \beta$-arrestin offshoot gaining the added property of GPCR recognition. The GPCR-regulated arrestins diverged from the rest of the clade very early in arrestin evolution. The visual arrestins appear to have evolved first and given rise to the $\beta$-arrestins. Visual arrestin has very high affinity and specificity for the rhodopsin GPCR, whereas cone arrestin has lower affinity and selectivity for rhodopsin and behaves much more like the $\beta$-arrestins. This has been postulated as an intrinsic mechanism to speed up rhodopsin recycling to increase photosensitivity and rapid adaptation to changing light conditions (Sutton et al., 2005).

The total sequence drift between the visual and $\beta$-arrestins is relatively small, whereas the greatest divergence is between $\alpha$ - and $\beta$-arrestins and the entire ARRDC clade (Fig. 1A) (Aubry et al., 2009). Although the visual and $\beta$-arrestins form a tight family of GPCRinteracting proteins, the $\alpha$-arrestins and VPS26 proteins have more divergent sequences and functions from each other. Analysis of the arrestin identity matrix shows the $\beta$-arrestins, $\alpha$-arrestins, and VPS26 proteins as three distinct families (Fig. 1B). However, ARRDP1 and ARRDP5 are quite distinct from all the other arrestins, having a maximum of $20 \%$ similarity to any other arrestin. It appears that all the arrestins should 

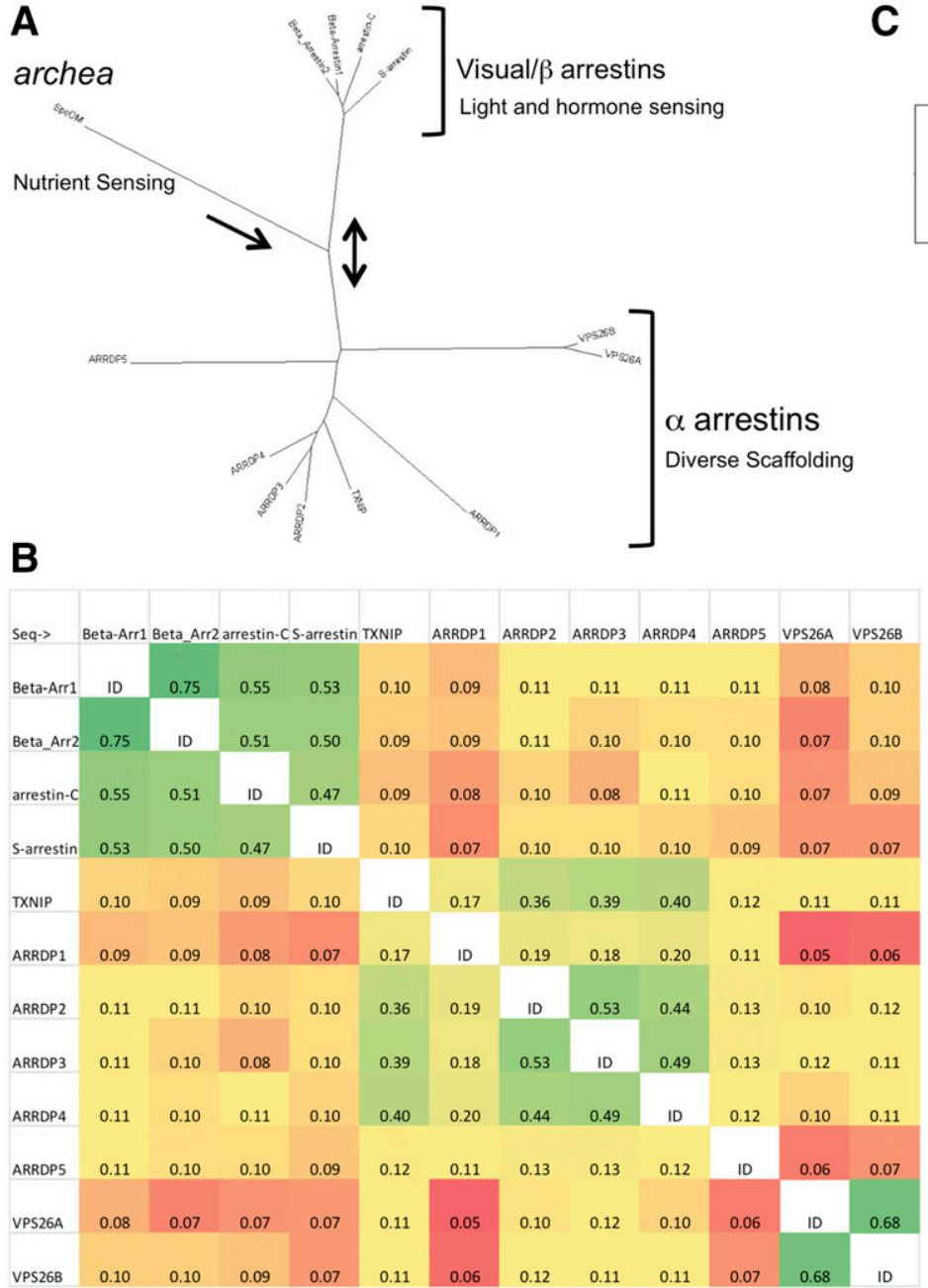

\section{B}

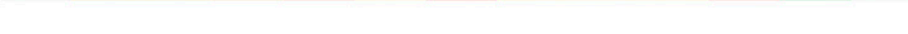

C

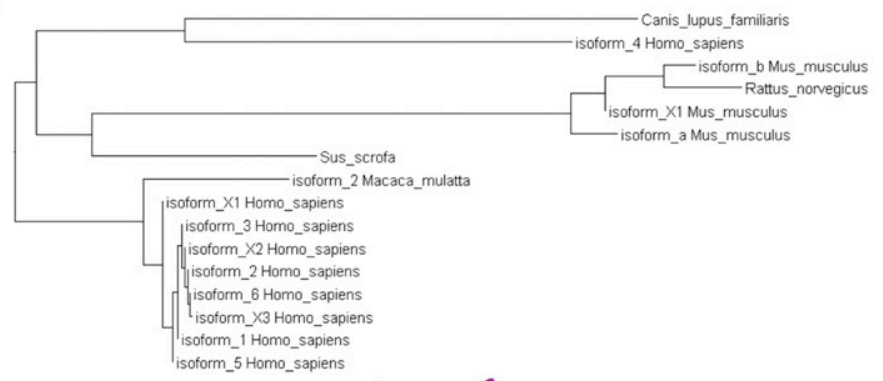

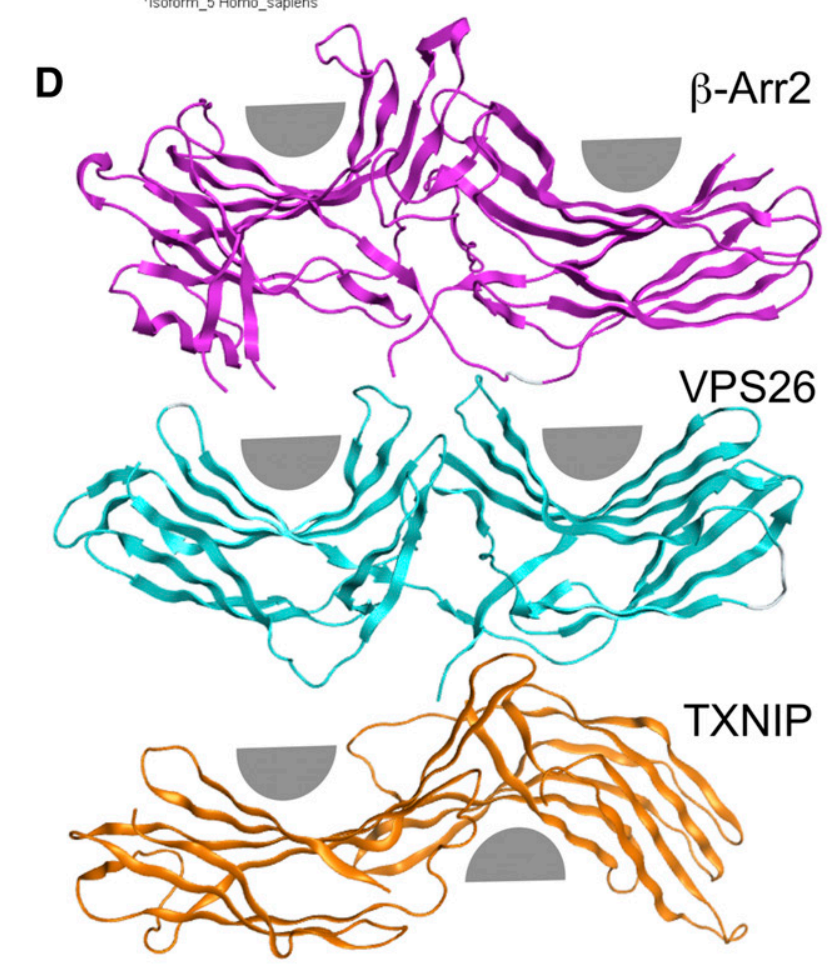

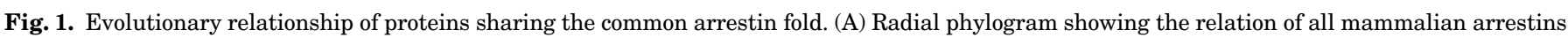

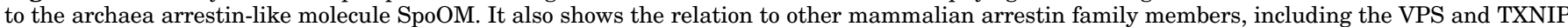

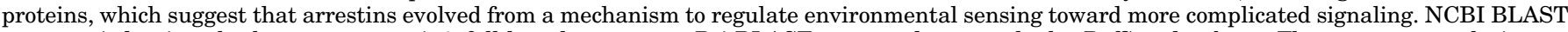

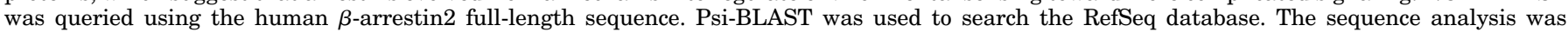

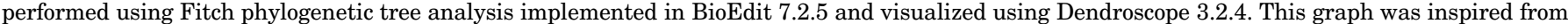

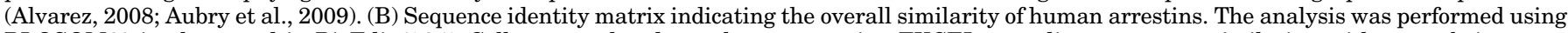

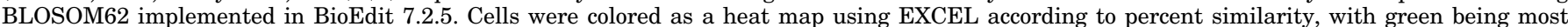

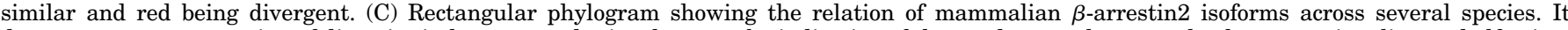

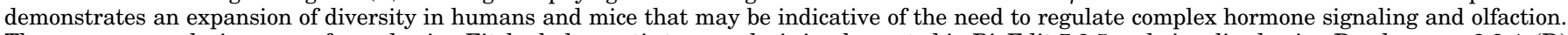

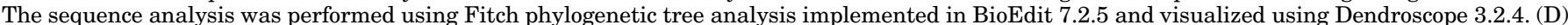

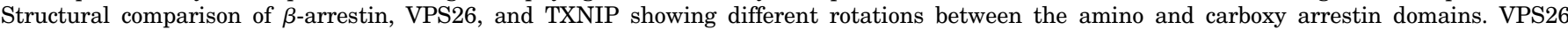

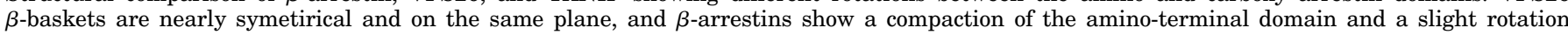

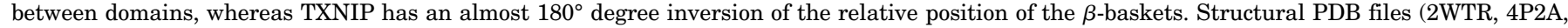

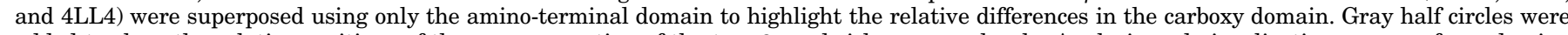

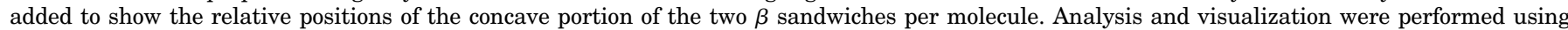
MOE 2014.09.

have the capacity to translocate, although translocation is not required for all of their known functions. The $\beta$-arrestins may be the only members of the clade whose functions are dependent upon translocation between multiple cellular compartments.

Illustrating the degree to which $\beta$-arrestins have evolved as specialized GPCR regulators, a close look at the $\beta$-arrestin 2 phylogeny in mammals shows a distinct speciation and branching of $\beta$-arrestin2 between rodents and humans (Fig. 1C). Compared with 1783 full-length genes encoding GPCRs in mice, there are only 799 in humans, with the bulk of the additional 1000 murine genes encoding olfactory receptors. It is thus tempting to speculate that there was evolutionary pressure on $\beta$-arrestins to provide for more nuanced regulation of environmental sensing GPCRs in rodents (Gloriam et al., 2007). The analysis supports the conclusion that human olfaction is very distinct from rodents and that GPCR and $\beta$-arrestin evolution are tightly linked and tied to the environmental necessities of each species (Haitina et al., 2009). 


\section{B. Arrestin Structure}

In a general sense, all arrestins are semi-bisymmetric soluble proteins that link plasma membrane-initiated signaling events to intracellular responses. Like most reversible signaling interactions, the affinities are relatively weak, allowing for more dynamic temporal scaffolding. A substantial body of X-ray crystallographic structural data is available for the visual $/ \beta$-arrestins (Table 1), whereas the $\alpha$-arrestins are represented by TXNIP and VPS26. The conserved arrestin fold that defines the family consists of a semi-symmetric fold of $20 \beta$ strands condensing into two $\beta$ strand sandwich structures, forming two baskets connected by a short hinge domain (Fig. 1D) (Vishnivetskiy et al., 2002; Aubry et al., 2009).

A number of domains important to arrestin function have been characterized, some universal, others unique to visual $/ \beta$-arrestins. There are three major recognized differences between the $\alpha$ - and visual/ $\beta$-arrestins. Visual $/ \beta$-arrestins possess an $\mathrm{N}$-terminal helix $\left(\mathrm{R}^{99} \mathrm{LQERLI}{ }^{105}\right.$ in bovine $\beta$-arrestin 2$)$ that has not appeared in $\alpha$-arrestin structures and whose sequence is unique to visual $/ \beta$-arrestins (Sutton et al., 2005) (Fig. 2 ). The $\mathrm{C}$ terminus of arrestins exhibits a switch from the $\alpha$-arrestin PPPxYS motif to the $\beta$-arrestin DDIVFE motif. Note that arrestin 1 and VPS26 diverge from the rest of their respective cohort in this region. Finally, the relative rotations of the two baskets are unique, implying different activation dynamics between $\alpha$ - and visual $/ \beta$-arrestins (Fig. $1 D$ ). In contrast, the polar core is conserved between $\alpha$ - and visual $/ \beta$-arrestins, suggesting that interaction with GPCRs or GPCR-like motifs is central in propagating structural changes. TXNIP has highest sequence identity with the $\alpha$-arrestins but structurally is most closely related to VPS26 proteins, with the greatest similarity in the $\mathrm{N}$ domain. Like visual $/ \beta$-arrestins, TXNIP has $20 \beta$ sheets divided into $\mathrm{N}$ and $\mathrm{C}$ domain concave baskets and a polar core. However, the N- and C-terminal domains of TXNIP are rotated away from each other (Polekhina et al., 2013; Hwang et al., 2014). Superposing a $\beta$-arrestin 1 structure [Protein Data Bank (PDB) file: 2 WTR] with TXNIP (PDB file: 4LL4), there is a $15.2 \AA$ root mean square deviation, whereas superposing a VPS26 (PDB file: 2FAU) structure with full-length TXNIP (PDB file: 4LL4) gives a $19.1 \AA$ root mean square deviation. Additionally, although in visual $/ \beta$-arrestins the inside of both of the baskets has a positive charge, in TXNIP the $\mathrm{N}$ domain is negative, whereas the $\mathrm{C}$ domain is positive.

Proceeding from the $\mathrm{N}$ to $\mathrm{C}$ terminus of visual/ $\beta$-arrestins, conserved structural elements include the N-terminal arm, Motif II, the phosphate sensor, the hinge domain, the gate loop/polar core, and the C-terminal arm that contains several protein-protein interaction motifs (Figs. 2 and 3, A-C).
1. N-Terminal Arm. Residues in the $\mathrm{N}$ terminus $\left(\mathrm{V}^{11} \mathrm{I}^{12} \mathrm{~F}^{13}\right.$ of bovine visual arrestin) are involved in stabilizing arrestin conformation, whereas the nearby residues $\mathrm{K}^{14}$ and $\mathrm{K}^{15}$ have been implicated in phosphate binding (Vishnivetskiy et al., 2000). Alanine substitution of residues 11-13 permits visual arrestin to bind to light-activated rhodopsin independent of receptor phosphorylation state, indicating a role in stabilizing the inactive conformation. Conversely, substitution of residues 14 and 15 dramatically impairs visual arrestin binding to phosphorylated light-activated rhodopsin, suggesting that the two lysines help direct receptorattached phosphates toward the phosphate sensor and participate in phosphate binding in the active state.

2. Finger Loop/Motif II. The next characterized subdomain is motif II, located in the finger loop, the uppermost loop (visual arrestin residues 68-79) on the N-terminal side of the hinge domain (Granzin et al., 1998). The conformational flexibility of motif II has been shown to be important for high-affinity receptor binding (Dinculescu et al., 2002; Vishnivetskiy et al., 2004). Spin-labeling and fluorescence quenching data indicate that in the unbound state motif II makes close contact with the arrestin $\mathrm{N}$ domain, but, when bound to phosphorylated light-activated rhodopsin, it is extended and buried in the rhodopsin-arrestin interface (Hanson et al., 2006a; Sommer et al., 2007). The crystal structure of p44 arrestin (Arr1-370A; PDB file: 3UGX), a naturally occurring splice variant with a truncation of the inhibitory $\mathrm{C}$ terminus that can terminate phototransduction by binding to nonphosphorylated lightactivated rhodopsin (Schroder et al., 2002), indicates that that rearrangement of motif II is a major change associated with constitutive arrestin activity (Granzin et al., 2012). Similarly, a crystal structure of lightactivated rhodopsin bound to a peptide analog of the finger loop of visual arrestin (PDB file: 4PXF) shows that the finger loop engages a binding crevice on the surface of the activated receptor in much the same manner as the G $\alpha$ t C terminus (Szczepek et al., 2014). Engaging the receptor stabilizes the finger loop structure in the form of a reverse turn.

3. Phosphate Sensor. Because visual $/ \beta$-arrestin function depends on its ability to detect a phosphorylated GPCR on the plasma membrane, the location of the phosphate sensor has been a major area of interest (Palczewski et al., 1991b; Gurevich and Benovic, 1993, 1997; Vishnivetskiy et al., 2011; Gurevich and Gurevich, 2013; Gimenez et al., 2014a). Although all visual $/ \beta$-arrestins can bind to unphosphorylated GPCR C-terminal peptides, different arrestin-GPCR pairs vary in their affinities for nascent versus phosphorylated receptor. Visual arrestin binding to rhodopsin is highly dependent on phosphorylation, leading to a rapid dissociation rate when bound to unphosphorylated light-activated rhodopsin, whereas $\beta$-arrestin binding to GPCRs is less phosphorylation-dependent, relying on 
TABLE 1

Exemplary arrestin PDBs and structural form

\begin{tabular}{|c|c|c|c|}
\hline PDB File & Structural Form & Arrestin State & References \\
\hline \multicolumn{4}{|l|}{$\alpha$-Arrestins } \\
\hline 4GEJ/4GEI & TXNIP & N-terminal domain & Polekhina et al., 2013 \\
\hline 4LL4/4LL1/4GFX & TXNIP-TRX complex & Bimolecular complex & Hwang et al., 2014 \\
\hline 2FAU & VPS26 & Monomer & Shi et al., 2006 \\
\hline $4 \mathrm{P} 2 \mathrm{~A}$ & VPS26A-SNX27 PDZ domain & Bimolecular complex & Gallon et al., 2014 \\
\hline \multicolumn{4}{|l|}{ Visual $/ \beta$-arrestins } \\
\hline 1AYR & Bovine rod arrestin1 & Homotetramer & Granzin et al., 1998 \\
\hline 1CF1 & Bovine rod arrestin 1 & Monomer & Hirsch et al., 1999 \\
\hline 3UGX/3UGU & Bovine $\mathrm{p} 44$ arrestin 1 & Monomer & Granzin et al., 2012 \\
\hline $4 \mathrm{~J} 2 \mathrm{Q}$ & Bovine p44 arrestin 1 & Active conformation & Kim et al., 2013 \\
\hline 1SUJ & Ambystoma tigrinum cone arrestin & Monomer & Sutton et al., 2005 \\
\hline $1 \mathrm{VQX} / 1 \mathrm{NZS}$ & Bovine arrestin $1-$ rhodopsin $\mathrm{C}$ terminus & Monomer & Kisselev et al., 2004a,b \\
\hline $4 \mathrm{PXF}$ & Bovine arrestin $1(67-77)$-retinal-free rhodopsin & Bimolecular complex & Szczepek et al., 2014 \\
\hline 4ZWJ & T4 lysozyme-rhodopsin-arrestin 1 chimera & Monomer & Kang et al., 2015 \\
\hline $1 \mathrm{G} 4 \mathrm{M}$ & Bovine $\beta$-arrestin 1 & C-terminal truncation & Han et al., 2001 \\
\hline 2WTR & Bovine $\beta$-arrestin 1 & Dimer & N/A \\
\hline 1JSY & Bovine $\beta$-arrestin 2 & Monomer & Milano et al., 2002 \\
\hline $3 \mathrm{P} 2 \mathrm{D}$ & Bovine $\beta$-arrestin 2 & Monomer & Zhan et al., 2011a \\
\hline $1 \mathrm{ZSH}$ & Bovine $\beta$-arrestin1-inositol hexakisphosphate & Bimolecular complex & Milano et al., 2006 \\
\hline 3GC3 & Bovine $\beta$-arrestin $1(1-385)$-clathrin & Bimolecular complex & Kang et al., 2009 \\
\hline 4JQI & Rat $\beta$-arrestin $1-\mathrm{V} 2$ receptor phosphopeptide-Fab & Trimolecular complex & Shukla et al., 2013 \\
\hline 2IV8 & Human $\beta$-arrestin2(317-410)-AP2 $\beta$-appendage & Bimolecular complex & Schmid et al., 2006 \\
\hline
\end{tabular}

contact with nonphosphorylated receptor elements to add stability to the interaction (Vishnivetskiy et al., 2011). The primary site of phosphorylated receptor interaction is on the positively charged concave surface of the two arrestin $\beta$-baskets. Phosphate-binding residues have been localized to three segments of visual arrestin, including residues 68-78, 170-182, and 330348 (Mokarzel-Falcon et al., 2008). Interestingly, the two $\beta$-baskets are not symmetrical, and the majority of direct receptor affinity appears to lie in the $\mathrm{N}$-terminal basket. The phosphate sensor, which is integral to controlling the polar core, is designed to be a chargeoperated trigger, with $\mathrm{R}^{175}$ and other amino acids within the 158-185 region driving arrestin activation though propagating structural rearrangements (Gurevich and Benovic, 1993; Kieselbach, et al., 1994; Vishnivetskiy et al., 2000; Han et al., 2001; Nobles et al., 2011). Mutations that destabilize the polar core by disrupting the phosphate sensor, e.g., $\mathrm{R}^{169} \mathrm{E}$ and $R^{175} \mathrm{D} / \mathrm{E}$, or removal of the auto-inhibitory $\mathrm{C}$ terminus, e.g., $\mathrm{D}^{383}$ STOP, generate constitutively active arrestins that lose the ability to recognize receptor phosphorylation while retaining selectivity for the ligand-activated receptor conformation (Gurevich and Benovic, 1997; Kovoor et al., 1999).

4. Hinge Domain. The arrestin hinge domain is formed by the conjunction of $\mathrm{N}$ - and C-terminal loops from each half of the protein that come together to create an area of regulated flexibility. The hinge region is defined primarily as the loops from motifs I and IV (Figs. 2 and 3, A-C) (Vishnivetskiy et al., 2002). The entire midsection of the arrestin molecule, which contains the polar core and the hinge, is composed of three loops from the N-terminal half and two loops from the C-terminal half of the upper surface, along with a series of crossing peptide sequences from the bottom face of the protein. This interface between the $\mathrm{N}$ and $\mathrm{C}$ domain is involved in interactions with the arrestin $\mathrm{N}$-terminal helix, GPCR domains, and microtubules.

5. Gate Loop and Polar Core. The polar core is essential to the activation of arrestin. In visual arrestin, rearrangement of the polar core is controlled primarily by the gate loop $\left(\mathrm{D}^{296}-\mathrm{N}^{305}\right)$, also known as the lariat loop (residues 282-309) (Han et al., 2001; Kim et al., 2013). The gate loop is so named because of its ability to stabilize the polar core using charge pair atoms from aspartic acid that interact with arginine in the polar core. The arrestin C terminus (residues 384-391) interacts with both the $\mathrm{N}$ terminus and areas on the C-terminal side of the hinge domain, stabilizing the inactive arrestin molecule to prevent structural rearrangements and blocking effector and specific hydrophobic binding sites (Gurevich and Benovic, 1993). Receptor binding displaces the arrestin $\mathrm{C}$ terminus, destabilizing the core and exposing new receptor interaction sites (Kim et al., 2013). A salient difference between visual arrestin and the nonvisual $\beta$-arrestins is that the interdomain hydrogen bonds that stabilize the polar core are weakened in $\beta$-arrestins, such that the hydrogen bond networks in $\beta$-arrestin 1 and 2 resemble that seen in the preactivated p44 arrestin1 structure (Granzin et al., 2012; Kim at al., 2013). This suggests the nonvisual arrestins may exist in a partially preactivated state, accounting for their lower dependence upon receptor phosphorylation compared with visual arrestin (Gurevich et al., 1995).

6. C-Terminal Arm. The major role of the arrestin C-terminal arm is to work with the gate loop to stabilize the inactive or closed confirmation of arrestin (Shukla et al., 2013). Additionally, the very C-terminal region is the area of highest divergence among the arrestin superfamily, indicating that this region has adapted to 


\section{Mammalian Arrestins}

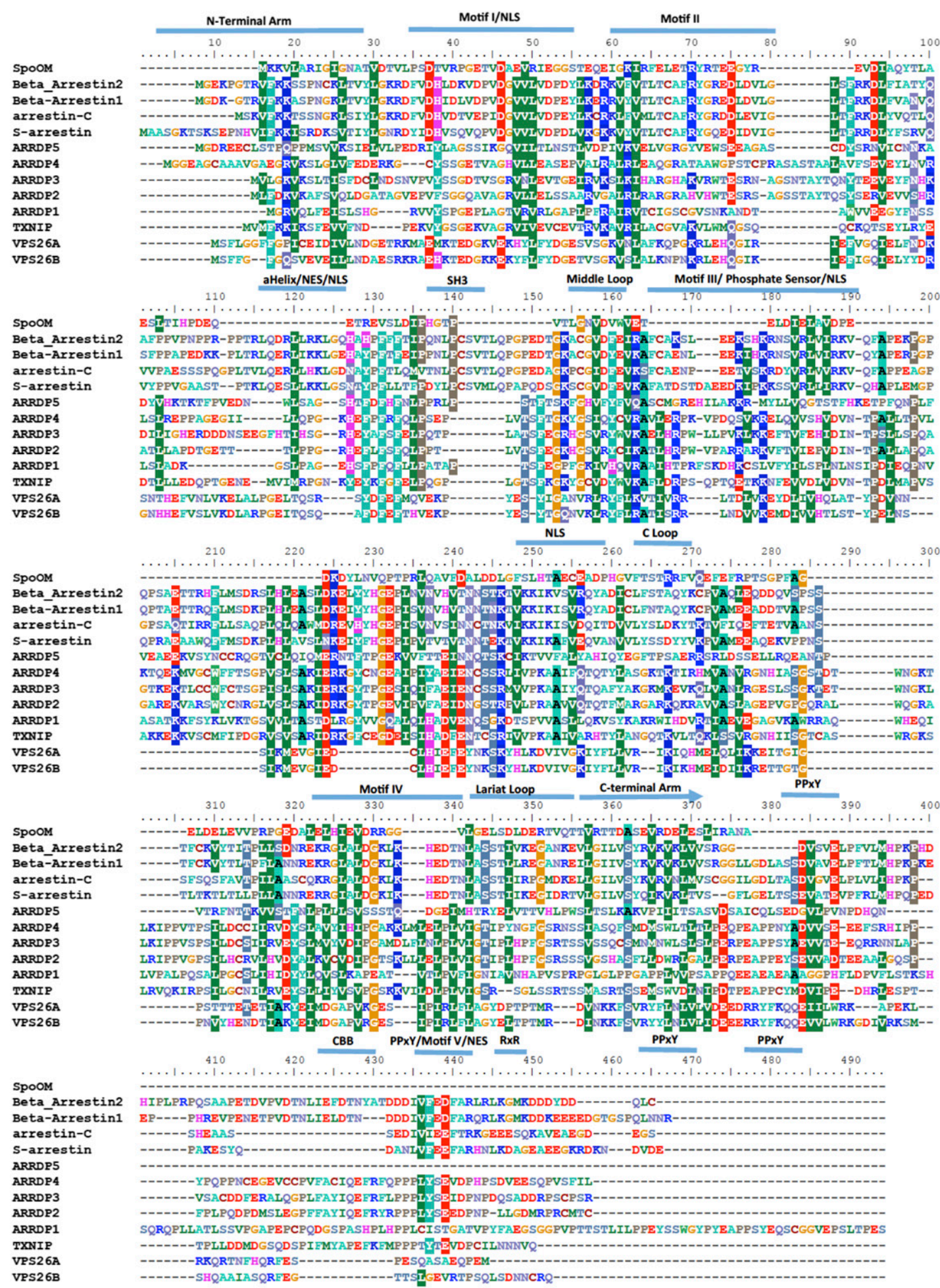

Fig. 2. Amino acid sequence alignment of arrestins indicating extensions, insertions, deletions, and functional domains among the arrestin clade. Domains depicted include the N- and C- terminal arms, Motif I-IV, NES, three NLS, SH3 domain, four PPxY motifs, clathrin binding domain (CBD), and the middle, C, and Lariat loops. The analysis was performed using ClustalW multiple alignment analysis implemented in BioEdit 7.2.5. Amino acids are colored according to their chemical properties, and conserved consensus resides are colored filled.

their divergent functions. The $\alpha$-arrestins ARRDC1 and ARRDC3 possess a PPxY motif within the $\mathrm{C}$ terminus that confers binding to WW proteins like the E3 ubiquitin ligase NEDD4 (Nabhan et al., 2010; Puca et al., 2013). In the nonvisual arrestins, the $\mathrm{C}$ terminus contains clathrin and AP-2-binding motifs that are essential for supporting GPCR endocytosis. Residues 89-100 of the clathrin heavy chain bind C-terminal 


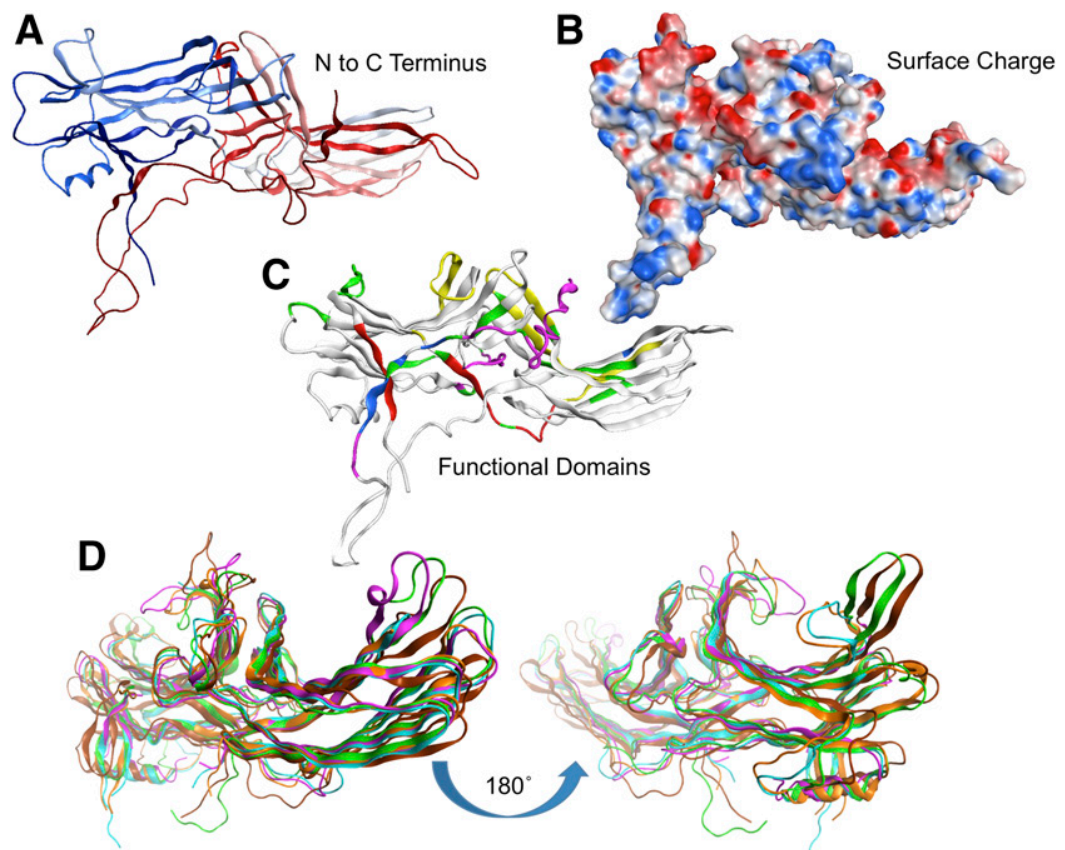

Fig. 3. Visual $/ \beta$-arrestin topologic structural analysis showing the overall tertiary fold of arrestins, the charge distribution surface, and the major functional and interaction domains. (A) Ribbon diagram indicating arrestin folding from $\mathrm{N}$ terminus (blue) to C terminus (red). (B) Surface diagram indicating the positive (red) and negative (blue) charge regions. (C) Functional domain diagram showing areas of functional importance from X-ray and mutagenesis studies. Domains are colored such that red regions are involved in receptor binding, green regions are involved in oligomerization, blue are important in arrestin activiation, and yellow regions interact with microtubules. Analysis and visualization were performed using MOE 2014.0. (D) Comparison of multiple X-ray crystal structures of $\beta$-arrestins shows plasticity and signaling diversity. The images show large conformational rearrangements localized to the outer loops, and the hinge domain proximal to the N-terminal domain. Note the disordered and unresolved loops present in the bottom of each image corresponding to the beginning of the C-terminal arm containing the CBD, Motif V, and RxR motifs. Topologic flexible regions of arrestin from PDB files 2WTR, 3GD1, 3GC3, 1AYR.A, and 1AYR.B. The two images are rotated $180^{\circ}$ to each other to show the amino or carboxy domains. Structures were aligned and superposed using all carbon $\alpha$ atoms. Each chain has a unique color. Analysis and visualization were performed using MOE 2014.09.

residues $373-377$ in $\beta$-arrestin2, which comprise a conserved LIEFE/LD motif shared by the $\beta$-arrestins (Goodman et al., 1996; Krupnick et al., 1997). This same region of $\beta$-arrestin 2 contains an RxR motif that binds the $\beta 2$-adaptin subunit of the AP-2 complex and promotes GPCR clustering in clathrin-coated pits (Laporte et al., 2000; Kim and Benovic, 2002; Schmid et al., 2006).

\section{Arrestin Activation}

Binding of a visual $/ \beta$-arrestin to the phosphorylated intracellular domains of an activated GPCR induces conformational rearrangements that enable it to perform its extracellular ligand-regulated scaffolding functions. Both static structural data from X-ray crystallography and dynamic data obtained using spin labels and resonance energy transfer probes offer insights into how arrestins are activated and how different receptors are able to initiate different arrestin functions.

Arrestin activation commences upon interaction with receptor-attached phosphates, a step that displaces the arrestin $\mathrm{C}$ terminus and destabilizes the polar core, priming the arrestin for a conformational rearrangement that enables high-affinity receptor binding (Schleicher et al., 1989; Schroder et al., 2002; Kirchberg et al., 2011; Gimenez et al., 2012a). The open activated conformation forms when the finger loop/motif II is stabilized into a fold that leaves the polar core exposed and allows interaction with receptor loops (Gurevich and Benovic, 1993). This is associated with rearrangements in motifs I, III, and IV, leading to dissociation of the hinge salt bridges and protein flexing (Granzin et al., 1998). Analysis of conformational shifts in $\beta$-arrestin 1 and 2 binding to rhodopsin in vitro, using double-electron electron resonance spectroscopy, confirms release of the $\mathrm{C}$-terminal tail and movement of the finger loop toward the predicted location of the receptor (Zhuo et al., 2014). The crystal structure of a preactivated C-terminally truncated variant of visual arrestin (Arr1-370A), which was activated during crystallization by incubation with retinal-free rhodopsin (PDB file: 4JQ2), shows a dramatic $21^{\circ}$ twist between the $\mathrm{N}$ - and C-terminal domains and local changes in loop conformation and hydrogen-bonding networks, compared with the structure of unactivated full-length visual arrestin (Kim et al., 2013). Similar findings were reported for a structure of $\beta$-arrestin 1 cocrystallized in the presence of a Fab fragment and a phosphopeptide corresponding to the last 29 amino acids of the V2 vasopressin receptor (PDB file: $4 \mathrm{JQI}$ ), where a $20^{\circ}$ rotation between the $\mathrm{N}$ - and $\mathrm{C}$-terminal domains is present compared with the structure of inactive $\beta$-arrestin1 (Shukla et al., 2013). Finally, the crystal 
structure of a fusion protein composed of T4 lysozyme, mutationally stabilized rhodopsin, and a preactivated visual arrestin mutant (PDB file: $4 \mathrm{ZWJ}$ ) demonstrates that arrestin activation involves a $20^{\circ}$ rotation between the $\mathrm{N}$ and $\mathrm{C}$ domains that opens a cleft in the arrestin surface to accommodate a short helix in the rhodopsin second intracellular loop (Kang et al., 2015). Figure 3D depicts an overlay of basal and activated arrestin structures, illustrating the major areas of conformational flexibility. This two-step model wherein arrestin first engages the phosphorylated GPCR C terminus, prompting a conformational rearrangement that enables it to dock with the heptahelical receptor core, has been visualized using single-particle negative-stain electron microscopy (Shukla et al., 2014).

The dynamics of arrestin recruitment and the corresponding conformational shifts in the arrestin molecule have been probed using resonance energy transfer. Intermolecular bioluminescence resonance energy transfer (BRET) between Renilla luciferase-tagged $\beta$-arrestin 2 and yellow fluorosecent protein-tagged GPCRs indicates that half-maximal association occurs within 1-2.5 minutes (at $25^{\circ} \mathrm{C}$ ) for arrestin recruitment to the receptor upon agonist stimulation (Charest and Bouvier, 2003; Charest, et al., 2005). Measured by intermolecular fluorescence resonance energy transfer (FRET), the process appears even faster, taking only seconds to occur (Nuber et al., 2016). Recruitment is followed closely in time by a conformational shift (Charest et al., 2005). Consistent with the model that arrestins undergo structural rearrangement after the phosphate sensor is triggered by phosphorylated receptor domains, the intramolecular shifts in $\beta$-arrestin 2 conformation upon binding the $\beta 2$-adrenergic receptor are slower than the initial binding $(\tau 1.2$ seconds for recruitment versus 2.2 seconds for conformational change) (Nuber et al., 2016).

The dynamic conformational shifts observed in $\beta$-arrestin 2 upon receptor stimulation vary, suggesting that information about ligand and receptor is encoded within the activated arrestin structure. When intramolecular fluorescent arsenical hairpin (FlAsH) FRET or BRET is used to report on arrestin conformational changes from multiple vantage points within the $\beta$-arrestin2 molecule, it is apparent that different GPCRs impose characteristic arrestin conformational signatures (Lee et al., 2016; Nuber et al., 2016). Interestingly, the $\beta$-arrestin2 FlAsH BRET signal at some positions relates to the avidity of GPCR-arrestin binding and at others correlates with arrestin engagement of downstream signals (Lee et al., 2016). Even different ligands binding the same GPCR influence arrestin conformation and function. When measured by intramolecular BRET between the $\beta$-arrestin2 $\mathrm{N}$ and $\mathrm{C}$ terminus (Charest et al., 2005), conventional GPCR agonists produce similar increases in BRET signal, whereas biased agonists that recruit arrestin without activating heterotrimeric $\mathrm{G}$ proteins trigger modest shifts in the opposite direction (Shukla et al., 2008). For a panel of biased angiotensin $\mathrm{AT}_{1 \mathrm{~A}}$ receptor peptides, the $\beta$-arrestin 2 FlAsH BRET signal detected from discrete positions within the $\mathrm{C}$-terminal domain correlates with the avidity of GPCR-arrestin binding measured by fluorescence recovery after photobleaching (Zimmerman et al., 2012; Lee et al., 2016). Although the factors, beyond ligand-induced changes in receptor structure, that dictate arrestin conformation are incompletely understood, reports that different GRKs specify arrestin signaling versus desensitization (Kim et al., 2005; Ren et al., 2005; Nobles et al., 2011; Zimmerman et al., 2012) suggest that the pattern of receptor phosphorylation may imprint a phosphorylation code that regulates arrestin function by influencing its activated conformation (Tobin et al., 2008; Liggett, 2011).

Once bound, the lifetime of the GPCR-arrestin complex is determined by several factors, including receptor structure (Oakley et al., 2000, 2001), ligand off-rate (Krasel et al., 2005), and post-translational modifications such as reversible arrestin ubiquitination (Shenoy et al., 2001, 2007, (2009; Shenoy and Lefkowitz, 2003, 2005) and phosphorylation (Lin et al., 1997, 1999; Khoury et al., 2014). Most GPCRs fall into one of two classes based on their selectivity for the two $\beta$-arrestin isoforms and the longevity of the receptor-arrestin interaction (Oakley et al., 2000). One, termed class A, exhibits higher affinity for $\beta$-arrestin 2 than $\beta$-arrestin 1 and forms transient receptor-arrestin complexes that dissociate soon after the receptor internalizes. These receptors are rapidly resensitized and recycled back to the plasma membrane. The other, class B group exhibits equivalent affinities for $\beta$-arrestin 1 and $\beta$-arrestin 2 and forms long-lasting receptor-arrestin complexes that remain intact as the receptor undergoes endosomal sorting. These receptors tend to be sequestered in endosomes and either recycle slowly or are degraded. Intriguingly, the conformational shift in $\beta$-arrestin 2 induced by binding to the class A $\beta 2$ adrenergic receptor persists for as much as 5 seconds after the receptor-arrestin complex itself dissociates (Nuber et al., 2016), suggesting that whereas class B GPCRs may tie up arrestins in stoichiometric signalsome complexes that distribute to endosomes, class A receptors might be able to act upon arrestins catalytically, much in the same manner as heterotrimeric $G$ proteins.

\section{The Arrestin-GPCR Interface}

Two facets of the visual $/ \beta$-arrestin-GPCR interaction that are critical to its function are the ability to interdict GPCR coupling to heterotrimeric G proteins, which is the physical basis for homologous GPCR desensitization, and the ability to recognize a multitude of activated GPCRs despite variations in their 
transmembrane domain orientation and intracellular loop (ICL) structure.

The former is accomplished through a shared GPCR interface. $G$ protein activation involves binding of the $\mathrm{G} \alpha$ subunit $\mathrm{C}$ terminus within a cytoplasmic crevice in the GPCR transmembrane bundle that opens upon receptor activation (Cherezov et al., 2007; Rasmussen et al., 2007, 2011a,b). The finger loop/motif II of all four visual $/ \beta$-arrestins contains a concensus sequence, $(\mathrm{E} / \mathrm{D}) \mathrm{x}(\mathrm{I} / \mathrm{L}) \mathrm{xxxGL}$, that is shared with the $\mathrm{C}$ termini of $\mathrm{G} \alpha \mathrm{i} / \mathrm{t}$ family heterotrimeric $\mathrm{G}$ proteins, and the crystal structure of a peptide analog of the finger loop of visual arrestin in complex with rhodopsin (PDB file: 4PXF) shows that $\mathrm{G}$ proteins and arrestins use a common interface (Szczepek et al., 2014). The T4 lysozymerhodopsin-visual arrestin chimera structure (PDB file: 4ZWJ) representing the active GPCR-arrestin complex identifies four principal rhodopsin-arrestin interface patches involving mostly the N-terminal arrestin basket (Kang et al., 2015). When fully engaged, the visual arrestin finger loop (residues 70-78) is stabilized as a short $\alpha$-helix that interacts with the $\mathrm{C}$ terminus of transmembrane domain (TM)7, the $\mathrm{N}$ terminus of helix 8 , and ICL1 of rhodopsin, whereas the adjacent arrestin $\beta$-strand (residues 79-86) makes contact with residues from TM5, TM6, and ICL3. Upon activation, the arrestin middle and C-loops move apart to accommodate rhodopsin ICL2, such that another interface patch is formed by the middle loop of the $\mathrm{N}$ domain (around residue 140) and the C-loop of the $\mathrm{C}$ domain (around residue 251) that interact with ICL2, and the arrestin back loop (residues 319-320) that contacts the C terminus of TM5. The N terminus of ICL2 contains the conserved DRY motif that is critical for regulating GPCR activation state, G protein coupling, and intracellular localization (Rovati et al., 2007; Kim et al., 2008c; Stambouli et al., 2014). Although not visualized in the crystal structure, additional contacts between the visual arrestin $\mathrm{N}$-terminal $\beta$-strand (residues 11-19) and the C-terminal tail of rhodopsin can be modeled (Kang et al., 2015). Whereas the structure of the unphosphorylated C terminus of GPCRs appears to be flexible, the nuclear magnetic resonance spectroscopy structure of a fully phosphorylated rhodopsin C terminus bound to visual arrestin shows a distinct structure imposed by very clear charge coupling between the negative receptor phosphates and the positively charged outer loop of the arrestin $\mathrm{N}$ domain basket (Kisselev et al., 2004a,b).

The GPCR selectivity of visual $/ \beta$-arrestins varies. Visual arrestin, which is expressed only in visual sensory tissue and exists primarily to desensitize rhodopsin, is highly dependent upon receptor phosphorylation and exhibits the largest differences in affinity between rhodopsin and other GPCRs (Gurevich et al., 1995). The ubiquitously distributed $\beta$-arrestin 1 and 2 , in contrast, which must regulate hundreds of different
GPCRs, have a weaker hydrogen-bonding network within the polar core that makes them less dependent upon receptor phosphorylation and more easily activated by diverse receptor structures (Gurevich et al., 1995). $\beta$-Arrestin2 is the least selective member of the visual $/ \beta$-arrestins in terms of GPCR interaction, and this is correlated with increased flexibility within the C-terminal basket (Zhan et al., 2011a). This area, which in most arrestins forms a stabilized $\beta$ sheet, has less defined secondary structure in $\beta$-arrestin 2 , probably reflecting greater flexibility to accommodate differences in GPCR structure.

Given that a few discrete contact patches form the GPCR-arrestin interface, it is not surprising that mutating a small number of residues can change the receptor selectivity of arrestins. Studies performed using visual arrestin $/ \beta$-arrestin 1 chimeras and alanine substitution mutagenesis have identified a handful of such receptor discriminator residues. Alanine substitution of the two highly conserved phosphate-sensing Lys residues in the visual arrestin $\mathrm{N}$ terminus $\left(\mathrm{K}^{14,15} \mathrm{~A}\right)$ reduces binding to active phosphorylated rhodopsin (Vishnivetskiy et al., 2000; Gimenez et al., 2012a), reflecting the dependence of visual arrestin-rhodopsin binding on receptor phosphorylation. In contrast, the analogous mutation in $\beta$-arrestin $2\left(\mathrm{~K}^{11,12} \mathrm{~A}\right)$ produces receptor-specific effects. For example, $\left[\mathrm{K}^{11,12} \mathrm{~A}\right]$ $\beta$-arrestin 2 is markedly impaired in binding to neuropeptide Y2 receptor, but not the closely related Y1 receptor (Gimenez et al., 2014a). Substitution of as few as 10 nonconserved residues located in two regions on the exposed surface of arrestins ( $\mathrm{N}$ domain residues 49-90 and $\mathrm{C}$ domain residues 237-268) markedly impairs the ability of visual arrestin, $\beta$-arrestin1, or $\beta$-arrestin2 to bind most GPCRs (Vishnivetskiy et al., 2011). Combining these two sets of mutations in $\beta$-arrestin 2 eliminates both predocking and agonist-induced recruitment to both neuropeptide $\mathrm{Y} 1$ and $\mathrm{Y} 2$ receptors (Gimenez et al., 2014a).

Even point mutations in the C-terminal central loop of $\beta$-arrestin2 (residues 230-260) dramatically affect GPCR selectivity. For example, the $\mathrm{Y}^{239} \mathrm{~T}$ mutation enhances $\beta$-arrestin 2 interaction with $\beta 2$-adrenergic receptor at the cost of affinity for M2 muscarinic, D1 dopamine, and D2 dopamine receptors. The $\mathrm{D}^{260} \mathrm{~K} / \mathrm{Q}^{262} \mathrm{P}$ mutant has the opposite effect, virtually eliminating $\beta 2$-adrenergic receptor binding, while preserving M2, $\mathrm{D} 1$, and $\mathrm{D} 2$ receptor binding, and the $\mathrm{Q}^{256} \mathrm{Y}$ mutant selectively reduces affinity for the $\mathrm{D} 2$ receptor. The $\mathrm{Y}^{239} \mathrm{~T} / \mathrm{Q}^{256} \mathrm{Y}$ mutation preserves $\mathrm{D} 1$ receptor binding while reducing affinity for the $\beta 2$-adrenergic and M2 receptors and eliminating D2 receptor binding (Gimenez et al., 2012b). For the Y1 and Y2 receptors, a $\mathrm{Y}^{238} \mathrm{~T}$ point mutation introduces several-fold selectivity for the Y1 over Y2 receptor, suggesting that arrrestins can be customized to preferentially recognize specific GPCR targets (Gimenez et al., 2014a,b). 
When fully engaged, arrestin and heterotrimeric G protein binding should be mutually exclusive. Yet some GPCRs, e.g., the type 1 parathyroid hormone (PTH), thyroid-stimulating hormone, type 1 sphingosine-1phosphate, and V2 vasopressin receptors, are able to mediate prolonged $\mathrm{G}$ protein-dependent signaling from within endosomal compartments even after undergoing arrestin-dependent internalization (Calebiro et al., 2009; Ferrandon et al., 2009; Mullershausen et al., 2009; Feinstein et al., 2013; Vilardaga et al., 2014). One proposal to account for this seeming paradox, supported by biophysical and single-particle negativestain electron microscopy data, is that some GPCRs can assemble signaling "megaplexes," composed of receptor, G protein, and arrestin (Thomsen et al., 2016). In this setting, the arrestin appears to linger in its initial binding mode, engaging the receptor C-tail, but failing to envelop the receptor intracellular domains, which would deny $G$ protein access to the receptor. The resulting complex continues to generate $G$ proteinmedicated signals while nonetheless undergoing arrestin-dependent redistribution into internalized vesicles.

\section{Visual/ $\beta$-Arrestins as Scaffolds}

\section{A. The Arrestin Interactome}

Beyond their traditional roles in GPCR desensitization/internalization, arrestins have been implicated in the control of multiple signaling processes. Early efforts to identify arrestin-binding partners using yeast two-hybrid or proteomic approaches uncovered myriad potential interactions. For example, one proteomicsbased screen reported that 337 distinct proteins coprecipitated with epitope-tagged $\beta$-arrestin 1 or 2 under varying conditions (Xiao et al., 2007). Although it is unlikely that so many proteins directly engage arrestins, it seems clear that arrestins do bind elements of several intracellular signaling cascades, and in many cases contribute to their positive or negative regulation (Luttrell and Gesty-Palmer, 2010). Table 2 summarizes many of the reported arrestin-dependent GPCR signaling pathways that have a degree of experimental validation. Viewed as a whole, arrestin signaling appears to encompass a fairly discrete set of functions, linking GPCRs to nonreceptor tyrosine kinases, MAP kinases (MAPKs), lipid kinases, protein phosphatases, ubiquitin ligases and deubiquitinating enzymes, enzymes involved in second-messenger degradation, and regulators of Ras-family small GTPases. Many of these putative effectors are not known to be regulated by heterotrimeric $G$ proteins, suggesting that arrestineffector pathways function in parallel with $\mathrm{G}$ proteineffector pathways to add dimensions to GPCR signaling.

1. Clathrin-Dependent Endocytic Machinery. The $\beta$-arrestins, but not the visual arestins, mediate clathrin-dependent sequestration of GPCRs (Ferguson,
2001). This is accomplished through regulated interaction of the $\beta$-arrestin $\mathrm{C}$ terminus with elements of the clathrin-coated pit following arrestin activation. As noted, arrestin activation initially involves interaction between the phosphate sensor and receptor-attached phosphates, leading to displacement of the $\mathrm{C}$ terminus. Once exposed, a conserved LIEFE/LD motif in the distal C terminus binds residues $89-100$ of the clathrin heavy chain (Goodman et al., 1996; Krupnick et al., 1997). Mutating $\mathrm{L}^{373}, \mathrm{I}^{374}$, and $\mathrm{F}^{376}$ of $\beta$-arrestin2 to alanine disrupts clathrin binding and markedly impairs GPCR sequestration (Goodman et al., 1997). Nearby the clathrin-binding motif is an $\mathrm{RxR}$ motif that binds the $\beta 2$ adaptin subunit of the AP-2 complex (Laporte et al., 2000; Kim and Benovic, 2002; Schmid et al., 2006). Mutating the $\beta$-arrestin $2 \mathrm{RxR}$ motif does not impair recruitment to the plasma membrane, but prevents $\beta 2$ adrenergic receptors from clustering in clathrin-coated pits.

2. Tubulin and Microtubules. All of the visual/ $\beta$-arrestins bind microtubules, with $\beta$-arrestin 1 and 2 showing the stronger interaction (Nair et al., 2004; Hanson et al., 2006b, 2007a). The site of interaction involves the concave sides of both the $\mathrm{N}$ and $\mathrm{C}$ domain baskets and substantially overlaps the receptor binding sites, but the affinity for microtubules is much lower than for activated GPCRs, allowing receptors to outcompete microtubules for arrestin binding. Interestingly, deletion mutations within the hinge region that reduce receptor binding enhance binding to microtubules, suggesting distinct microtubule-bound and receptorbound conformations (Vishnivetskiy et al., 2002; Hanson et al., 2007a).

3. Phosphoinositides. Several species of phosphoinositide bind to visual $/ \beta$-arrestins, of which the abundant cytosolic phosphoinositide, inositol hexakisphosphate (IP6), has the highest affinity (Palczewski et al., 1991a; Gaidarov et al., 1999). Two independent IP6 binding sites have been identified in $\beta$-arrestin 1 both by mutational analysis and X-ray crystallography, a lowaffinity site in the $\mathrm{N}$ domain involving residues $\mathrm{K}^{157}$ $\mathrm{K}^{160} \mathrm{R}^{161}$, and a high-affinity site in the $\mathrm{C}$ domain involving residues $\mathrm{K}^{232} \mathrm{R}^{236} \mathrm{~K}^{250} \mathrm{~K}^{324} \mathrm{~K}^{326}$ (Gaidarov et al., 1999; Milano et al., 2006). IP6 binding promotes $\beta$-arrestin1 self-association and is involved in receptor clustering in clathrin-coated pits and their subsequent internalization. Similarly, Drosophila visual arrestin contains a C-terminal domain IP6 binding site that when mutated interferes with arrestin trafficking in photoreceptor cells and light adaptation (Lee et al., 2003). In contrast, visual arrestin binding to IP6 involves principally the $\mathrm{N}$ domain residues $\mathrm{K}^{163} \mathrm{~K}^{166}$ $\mathrm{K}^{167}$. Unlike $\beta$-arrestin1, IP6 inhibits both selfassociation and its recruitment to light-activated rhodopsin (Hanson et al., 2006a; Zhuang et al., 2010). Solution NMR studies indicate that IP6 binding leads to release of the visual arrestin C-tail, presumably 
TABLE 2

The nonreceptor arrestin interactome

\begin{tabular}{|c|c|c|c|}
\hline Effector & Arrestin & Reported Function & References \\
\hline Clathrin heavy chain & $\beta$-Arrestin 1 & Clathrin-dependent GPCR endocytosis & Goodman et al., 1996 \\
\hline \multirow[t]{3}{*}{$\beta 2$-adaptin subunit of AP-2 } & $\beta$-Arrestin 2 & & Krupnick et al., 1997 \\
\hline & & & Laporte et al., 1999 \\
\hline & & & Laporte et al., 2000 \\
\hline \multirow[t]{3}{*}{ Tubulin; microtubules } & Arrestin1 & Sequestration of arrestin & Hanson et al., 2006b \\
\hline & $\beta$-Arrestin1 & Attenuation of MAPK activity & Hanson et al., 2007a \\
\hline & $\begin{array}{l}\beta \text {-Arrestin } 2 \\
\text { Arrestin } 4\end{array}$ & $\begin{array}{l}\text { Enhanced ubiquitination of cytoskeletal } \\
\text { proteins }\end{array}$ & \\
\hline \multirow[t]{4}{*}{$\mathrm{Ca}^{2+}$-calmodulin } & Arrestin 1 & Cytosolic sequestration of $\mathrm{Ca}^{2+}$-calmodulin & Wu et al., 2006 \\
\hline & $\beta$-Arrestin 1 & & \\
\hline & $\beta$-Arrestin 2 & & \\
\hline & Arrestin4 & & \\
\hline \multirow[t]{4}{*}{ Inositol hexakisphosphate } & Arrestin1 & Arrestin oligomerization & Palczewski et al., 1991a \\
\hline & $\beta$-Arrestin1 & Rhodopsin binding & Gaidarov et al., 1999 \\
\hline & $\beta$-arrestin 2 & Receptor endocytosis & Milano et al., 2006 \\
\hline & Arrestin4 & Arrestin nuclear translocation & Hanson et al., 2008 \\
\hline Src family tyrosine kinases & Arrestin1 & ERK1/2 activation & Luttrell et al., 1999; DeFea et al., 2000a \\
\hline \multirow{6}{*}{ c-Src; c-Yes; c-Hck; c-Fgr; c-Fyn } & $\beta$-Arrestin 1 & Dynamin 1 phosphorylation & Miller et al., 2000 \\
\hline & $\beta$-Arrestin 2 & Exocytosis/Granule release & Barlic et al., 2000; Imamura et al., 2001 \\
\hline & & Phosphorylation/Destabilization of GRK2 & Penela et al., 2001 \\
\hline & & FAK phosphorylation & Galet and Ascoli, 2008 \\
\hline & & EGF receptor transactivation & Noma et al., 2007 \\
\hline & & Phosphorylation of $\beta 2$ adaptin subunit of AP-2 & $\begin{array}{l}\text { Fessart et al., 2007; Zimmerman } \\
\quad \text { et al., } 2009\end{array}$ \\
\hline \multirow[t]{6}{*}{ c-Raf1-MEK1/2-ERK1/2 } & $\beta$-Arrestin1 & Activation of cytosolic ERK1/2 & DeFea et al., 2000b; Luttrell et al., 2001 \\
\hline & $\beta$-Arrestin 2 & Receptor internalization and trafficking & Lin et al., 1999; Khoury et al., 2014 \\
\hline & & p90RSK phosphorylation & Seta et al., 2002 \\
\hline & & Actin cytoskeletal reorganization/chemotaxis & Ge et al., 2003 \\
\hline & & ERK1/2-dependent transcription & Gesty-Palmer et al., 2005 \\
\hline & & $\begin{array}{l}\text { Mnk1/eIF4E phosphorylation/protein } \\
\text { translation }\end{array}$ & DeWire et al., 2008 \\
\hline
\end{tabular}

\section{ASK1-MKK4-JNK3}

\section{ASK1-MKK3/7-p38 MAPK}

$\mathrm{I} \kappa \mathrm{B} \alpha-\mathrm{I} \kappa \mathrm{B}$ kinase $\alpha / \beta$

Casein kinase II

cAMP phosphodiesterases PDE4D3; PDE4D5

Diacylglycerol kinases

PI 4-phosphate 5-kinase I $\alpha$

Phosphatidylinositol 3-kinase PTEN

\section{PP2A-Akt-GSK3 $\beta$}

Phospholipase A2

Nitric oxide synthases

Cofilin; chronophin; LIM kinase Filamin A

SHP-1; SHP-2

E3 ubiquitin ligases Mdm2; parkin; Nedd4; AIP4; TRAF6

Ubiquitin-specific protease 33

$\beta$-Arrestin2

$\mathrm{Na}^{+} / \mathrm{H}^{+}$exchanger1

Ral-GDS

$\beta$-Arrestin2

$\beta$-Arrestin2

$\beta$-Arrestin 1

$\beta$-Arrestin2

$\beta$-Arrestin1

$\beta$-Arrestin2

$\beta$-Arrestin 1

$\beta$-Arrestin2

$\beta$-Arrestin1

$\beta$-Arrestin1

$\beta$-Arrestin2

$\beta$-Arrestin2

$\beta$-Arrestin1 $\beta$-Arrestin1

$\beta$-Arrestin2

$\beta$-Arrestin2 $\beta$-Arrestin1 $\beta$-Arrestin2 $\beta$-Arrestin2 Arrestin1

$\beta$-Arrestin1 $\beta$-Arrestin2
$\beta$-Arrestin2 Activation of cytosolic JNK3

Sequestration of JNK outside the nucleus

$\beta$-Arrestin1 Scaffolding/Activation of p38 MAPK

$\beta$-Arrestin2 Inhibition of p38 MAPK

$\beta$-Arrestin1 Attenuation of $\mathrm{NF}_{\kappa} \mathrm{B}$ signaling

Activation of CK2

Attenuation of cAMP signaling

Attenuation of PKC signaling

Control of clathrin-dependent GPCR internalization

Localized inhibition of PI3K

Inhibition of AKT signaling and cell proliferation

Increased cell migration

Inactivation of Akt/GSK3 $\beta$

Activation of $\beta$-catenin signaling

Activation of Akt

Vasodilation and cutaneous flushing

Suppression of stress-induced iNOS transcription

Post-translational activation of iNOS eNOS-dependent S-nitrosylation of $\beta$-arrestin2 Actin cytoskeletal reorganization/chemotaxis Membrane ruffling

Inhibition of NK cell cytotoxicity

Ubiquitination of $\beta$-arrestin2

Stabilization of GPCR-arrestin complex

Increased p53-mediated apoptosis

Inhibition of Toll-like receptor signaling

Stabilization of GPCR-arrestin-ERK1/2 signalsome

GPCR ubiquitination and downregulation

Deubiquitination of $\beta$-arrestin2

Control of GPCR internalization

$\beta$-Arrestin1 Ubiquitination of NHE1 by Nedd 4

Negative regulation of NHE1 activity

$\beta$-Arrestin1 Cytoskeletal reorganization/granule exocytosis
McDonald et al., 2000; Song et al., 2006

Breitman et al., 2012

Sun et al., 2002; Yang et al., 2012

Zhao et al., 2004

Gao et al., 2004; Witherow et al., 2004

Kendall et al., 2011

Perry et al., 2002; Baillie et al., 2007

Nelson et al., 2007

Nelson et al., 2008

Wang and Defea, 2006

Lima-Fernandes et al., 2011

Beaulieu et al., 2005

Beaulieu et al., 2008

Kendall et al., 2011

Walters et al., 2009

Tan et al., 2015

Kuhr et al., 2010

Ozawa et al., 2008

Zoudilova et al., 2007, 2010

Scott et al., 2006

Yu et al., 2008

Shenoy et al., 2001

Shenoy and Lefkowitz, 2003

Wang et al., 2003

Wang et al., 2006

Shenoy et al., 2007

Bhandari et al., 2007; Shenoy, et al., 2008 Shenoy et al., 2009

Simonin and Fuster, 2010

Bhattacharya et al., 2002 
TABLE 2-Continued

\begin{tabular}{|c|c|c|c|}
\hline Effector & Arrestin & Reported Function & References \\
\hline ARF-GAP21 & $\beta$-Arrestin1 & $\begin{array}{l}\text { RhoA activation } \\
\text { Membrane ruffling }\end{array}$ & \\
\hline ARF6-ARNO & $\begin{array}{l}\beta \text {-Arrestin1 } \\
\beta \text {-Arrestin2 }\end{array}$ & GPCR endocytosis & Claing et al., 2001; Houndolo et al., 2005 \\
\hline N-ethylmaleimide-sensitive factor & $\beta$-Arrestin1 & Control of GPCR internalization & McDonald et al., 1999 \\
\hline Dishevelled 2 & $\beta$-Arrestin1 & Frizzled endocytosis and Wnt signaling & Chen et al., 2004 \\
\hline Kif3A kinesin motor protein & $\beta$-Arrestin1 & Targeting and internalization of Smoothened & Chen et al., 2004 \\
\hline Histone acetyltransferase p300 & $\beta$-Arrestin1 & Transcription of p 27 and c-Fos & Kang et al., 2005 \\
\hline Enchancer of zeste homolog 2 & $\beta$-Arrestin1 & $\begin{array}{l}\text { Promote histone } \mathrm{H} 4 \text { acetylation of } \mathrm{BCR} / \mathrm{ABL} \\
\text { Stimulate chronic myelogenous } \\
\text { leukemia progression }\end{array}$ & Qin et al., 2014 \\
\hline YY1 transcription factor & $\beta$-Arrestin1 & Repression of cdx4-hox transcription & Yue et al., 2009 \\
\hline $\operatorname{PPAR} \gamma$ & $\beta$-Arrestin1 & Repression of PPAR $\gamma-\mathrm{RXR} \alpha$ transcription & Zhuang et al., 2011 \\
\hline STAT1-TC45 & $\beta$-Arrestin1 & Dephosphorylation/Inactivation of STAT1 & Mo et al., 2008 \\
\hline
\end{tabular}

BCR, B cell receptor; FAK, focal adhesion kinase; eNOS, endothelial nitric oxide synthase.

by mimicking the multiphosphorylated rhodopsin $\mathrm{C}$ terminus.

4. $\mathrm{Ca}^{2+}$-Calmodulin. All four visual $/ \beta$-arrestins also bind $\mathrm{Ca}^{2+}$-liganded calmodulin with micromolar affinity (Wu et al., 2006). The binding site is on the concave side of the $\mathrm{C}$ domain basket, again overlapping the receptorand microtubule-binding surface, such that $\mathrm{Ca}^{2+}$-calmodulin can only interact with free cytosolic arrestin.

5. Src Family Nonreceptor Tyrosine Kinases. Several Src family tyrosine kinases have been reported to bind visual $/ \beta$-arrestins, including c-Src, c-Fgr, c-Fyn, c-Hck, and c-Yes (Luttrell et al., 1999; Barlic et al., 2000; DeFea et al., 2000a; Galet and Ascoli, 2008). As with many non-GPCR arrestin-binding partners, the sites of interaction have not been mapped with any degree of precision, and appear to involve several elements of both proteins. The $\mathrm{N}$ domain of $\beta$-arrestin 1 is proline rich and contains three $\mathrm{PxxP}$ motifs that interact with the Src homology (SH)3 domain of c-Src (Luttrell et al., 1999), but additional contacts with the c-Src SH1 (catalytic) domain are also involved (Miller et al., 2000). Additionally, visual arrestin binding to c-Src appears to involve the $\mathrm{SH} 2$ domain (Ghalayini et al., 2002). The arrestin-Src interaction appears to be constitutive, and it is not known whether arrestindependent recruitment of Src to GPCRs results in its activation, but immunostaining for the activated $\left(\mathrm{Y}^{530}\right.$ dephosphorylated) form of c-Src indicates that c-Src in the GPCR-arrestin complex is active (Luttrell et al., 1999).

6. Mitogen-Activated Protein Kinases. One of the better understood arrestin-signaling functions is scaffolding of MAPK cascades. The ubiquitous MAPKs play critical roles in cell cycle regulation/proliferation and survival/apoptotic signaling by controlling phosphorylation of nuclear transcription factors, e.g., Elk1 and c-Jun, as well as diverse regulatory functions mediated through phosphorylation of cytosolic substrates (Davis, 2000; Kyriakis and Avruch, 2001; Pearson et al., 2001). Each MAPK module consists of three kinases: MAPK kinase kinase, MAPK kinase, and MAPK, which must phosphorylate one another in succession, and arrestins play a traditional scaffold protein role, binding the component kinases to regulate the efficiency, fidelity, and compartmentalization of signaling (Luttrell and Miller, 2013). Significantly, although arrestins appear to engage all three major MAPK modules, ERK1/2, c-Jun N-terminal kinase (JNK), and p38 MAPK, the direction of regulation, positive or negative, varies. This most likely reflects the differing functions of MAPK modules and a consistent role of arrestins to favor activation of proliferative/survival pathways and suppression of apoptotic signals (Gurevich and Gurevich, 2013).

ERK1/2 activity is required for G0-G1 cell cycle transition and the passage of cells through mitosis or meiosis (Pearson et al., 2001). All three kinases of the ERK1/2 MAPK module, cRaf1-MAPK/ERK kinase (MEK) $1 / 2$-ERK1/2, bind $\beta$-arrestin $1 / 2$ individually and collectively (Luttrell, et al., 2001; Meng et al., 2009). In vitro, all three kinases can interact with either the isolated $\mathrm{N}$ - or C-terminal domains (Song et al., 2009a), suggesting a broad interaction surface involving the cytoplasmic surface of both domains, although the single $\mathrm{R}^{307} \mathrm{~A}$ point mutation in $\beta$-arrestin 1 is sufficient to reduce c-Raf1 binding and prevent arrestin scaffolding of the cascade (Coffa et al., 2011a). The ERK1/2 cascade is unique among the MAPK modules engaged by arrestins in that receptor activation regulates its assembly (Luttrell et al., 2001; Coffa et al., 2011b). Active ERK2 exhibits the highest affinity for receptorbound arrestin, with virtually no binding to free cytosolic protein. C-Raf1 also shows a preference for the active arrestin conformation, whereas MEK1 binds equally to active and inactive arrestins. Interestingly, a constitutively inactive arrestin mutant that mimics the microtubule-bound conformation binds ERK1/2 and c-Raf1 better than the inactive cytosolic form, leading to recruitment of inactive ERK1/2 to microtubules, where it is sequestered away from membrane-generated activating signals (Hanson et al., 2007a; Coffa et al., 2011b). Thus, arrestin scaffolds perform the dual roles of 
dampening basal ERK1/2 pathway activity, while specifically promoting the assembly of GPCR-associated signalsome complexes.

JNK family MAPKs play a major role in cellular stress responses and are involved in the normal physiologic processes of cell proliferation, apoptosis, differentiation, and migration (Davis, 2000; Kyriakis and Avruch, 2001). JNK1 and JNK2 are ubiquitously expressed, whereas JNK3 expression is largely confined to neuronal tissues. As with the ERK1/2 MAPK module, arrestins bind the component kinases of the neuronal JNK3 cascade, apoptosis signal regulating kinase (ASK) 1-MAPK kinase (MKK) 4/7-JNK3 (McDonald et al., 2000), but the consequences for JNK pathway regulation are much different. Similar to the ERK1/2 cascade, all four visual $/ \beta$-arrestins can bind ASK1MKK4/7-JNK3 in vitro (Song et al., 2009a). Both the $\mathrm{N}$ and $\mathrm{C}$ domains contribute to the JNK3-binding surface, although a 25-amino-acid stretch within the $\mathrm{N}$ domain exhibits the highest affinity for JNK3 $\alpha 2$ (Zhan et al., 2014). Phosphorylation of JNK3 by both MKK4 and MKK7 is required for full activation. In vitro, $\beta$-arrestin 2 interacts directly with MKK7 to phosphorylate JNK3 $\alpha 2$ (Zhan et al., 2013). JNK3 binding causes the arrestin to lose affinity for MKK7 and gain affinity for MKK4, indicating that the pathway components actually regulate one another's interaction with the scaffold. $\beta$-Arrestin2-dependent activation of MKK4-JNK3 has been reconstituted using purified proteins in vitro, and the efficiency of JNK3 activation demonstrates the biphasic dependence on arrestin concentration that is characteristic of a true scaffold protein (Zhan et al., 2011b, 2013). Interestingly, although all arrestins bind JNK3, only $\beta$-arrestin 2 is able to efficiently scaffold pathway activation. This was initially attributed to the presence of a consensus MAPK-docking motif, RRSLHL, in the $\beta$-arrestin2 C-terminal domain that conferred JNK3 binding and scaffolding activity when substituted for the corresponding sequence in $\beta$-arrestin1 (Miller et al., 2001). However, this motif is unique to rat $\beta$-arrestin2, whereas the capacity to activate JNK3 is shared with other mammalian $\beta$-arrestin 2 proteins. The two $\beta$ arrestin isoforms exhibit similar affinities for ASK1, MKK4, and JNK3, but apparently only $\beta$-arrestin2 is able to orient the kinases so as to support efficient phosphorylation (Seo et al., 2011). Bovine $\beta$-arrestin2 can also bind the ubiquitous JNK isoforms, JNK1 and JNK2, and support their activation by ASK1 and MKK4 or MKK7 in cells, suggesting the possibility that the activity of all three JNK isoforms can be modulated by arrestins (Kook et al., 2013).

The p38 MAPKs comprise the other major family of MAPKs. Like the JNKs, they are activated primarily in response to cell stress and proinflammatory signals (Kyriakis and Avruch, 2001, 2012) and are controlled via a modular ASK1-MKK3/6-p38MAPK phosphorylation cascade. Although the molecular mechanisms of activation have not been characterized in any detail, several studies have reported arrestin-dependent regulation of the p38 MAPK cascade (Luttrell and Miller, 2013).

7. Regulators of Nuclear Factor $\kappa B$ Signaling. $\beta$-Arrestin 1 and 2 both bind the $\mathrm{NF}_{\kappa} \mathrm{B}$ inhibitor, $\mathrm{I} \kappa \mathrm{B} \alpha$, and attenuate basal $\mathrm{NF}_{\kappa} \mathrm{B}$ signaling (Gao et al., 2004). In the cytoplasm, $\mathrm{NF}_{\kappa} \mathrm{B}$ dimers are bound to $\mathrm{I} \kappa \mathrm{B}$. In response to inflamatory signals, $\mathrm{I} \kappa \mathrm{B}$ is phosphorylated by $\mathrm{I} \kappa \mathrm{B}$ kinase, causing it to undergo proteosomal degradation. Once dissociated from $\mathrm{I} \kappa \mathrm{B}, \mathrm{NF}_{\kappa} \mathrm{B}$ translocates to the nucleus, where it promotes transcription of proinflammatory genes (Karin and Ben-Neriah, 2000). The $\mathrm{N}$-terminal domain of $\beta$-arrestin 2 , within residues $1-60$, binds to the C-terminal 40-amino-acid residues of $\mathrm{I} \kappa \mathrm{B} \alpha$, preventing its phosphorylation and subsequent degradation in response to stress signals like UV irradiation or activation of Toll-like receptor (TLR) 4 by bacterial lipopolysaccharide (Luan et al., 2005; Fan et al., 2007). Stimulation of $\beta 2$-adrenergic receptors enhances the $\beta$-arrestin $2-\mathrm{I} \kappa \mathrm{B} \alpha$ interaction, enhancing the stabilization of $\mathrm{I} \kappa \mathrm{B} \alpha$ and inhibiting $\mathrm{NF}_{\kappa} \mathrm{B}-$ dependent transcription. The $\mathrm{I} \kappa \mathrm{B}$ kinases, $\mathrm{I} \kappa \mathrm{B}$ kinase $\alpha$ and $\beta$, also coprecipitate with $\beta$-arrestins, and downregulation of $\beta$-arrestin 1 , but not $\beta$-arrestin 2 , enhances basal $\mathrm{NF}_{\kappa} \mathrm{B}$ transcriptional activity in HeLa cells (Witherow et al., 2004). In addition, interaction between $\beta$-arrestin 2 and the non-GPCR type III transforming growth factor- $\beta$ receptor negatively regulates $\mathrm{NF}_{\kappa} \mathrm{B}$ transcriptional activity in breast cancer cells (You et al., 2009).

In some circumstances, however, $\beta$-arrestin 1 can function as an enhancer of GPCR-stimulated $\mathrm{NF} \kappa \mathrm{B}$ transcription. $\beta$-arrestin 1 has a bipartite nuclear localization sequence (NLS) located between residues 157161 and $169-170$ in the $\mathrm{N}$ domain that allows it to bind importin $\beta 1$ and engage the nuclear import machinery (Hoeppner et al., 2012). Introducing a $\mathrm{K}^{157} \mathrm{~A}$ mutation blocks importin binding and prevents bradykinin receptor-mediated nuclear translocation of $\beta$-arrestin 1 . Inside the nucleus, $\beta$-arrestin 1 forms a complex with the p65/RelA subunit of $\mathrm{NF}_{\kappa} \mathrm{B}$. Arrestin binding facilitates p65/RelA acetylation by acetyltransferase CREB binding protein and phosphorylation by nuclear MSK1, two post-translational modifications that stabilize its DNA binding. As a result, cells expressing the $\mathrm{K}^{157} \mathrm{~A}$ $\beta$-arrestin1 mutant exhibit less p65/RelA promoter binding and a decrease in bradykinin-stimulated transcription of $\mathrm{NF}_{\kappa} \mathrm{B}$ targets like IL- $1 \beta$.

8. Casein Kinase II. Casein kinase II is a ubiquitously expressed, constitutively active Ser/Thr protein kinase that performs diverse functions related to cell survival and tumorigenesis. The catalytic subunit of casein kinase II was identified in a proteomic screen of $\beta$-arrestin2-binding proteins as well as a phosphoproteomic screen of angiotensin $\mathrm{AT}_{1 \mathrm{~A}}$ receptormediated phosphorylation following stimulation with 
the arrestin pathway-selective biased angiotensin analog, $\mathrm{Sar}^{1} \mathrm{Ile}^{4} \mathrm{Ile}^{8}$-AngII (Xiao et al., 2010; Kendall et al., 2011).

9. cAMP Phosphodiesterases. $\beta$-Arrestins 1 and 2 interact with all five type $4 \mathrm{D}$ isoforms of cAMP phosphodiesterase, PDE4D1-5 (Perry et al., 2002). The common catalytic subunit of PDE4D5 interacts with $\beta$-arrestin2 via interactions with the cytoplasmic surface of both the $\mathrm{N}$ domain, near residues 18-26, and $\mathrm{C}$ domains, near residues 215-220 and 286-291 (Baillie et al., 2007). The main function of arrestin-scaffolded PDE4 appears to be to enhance the negative regulation of $G$ protein signaling by accelerating second-messenger degradation.

10. Diacylglycerol Kinases. Both $\beta$-arrestins associate with the $\alpha, \beta, \gamma, \delta, \epsilon, \zeta$, or $\iota$ isoforms of diacylglycerol kinase, via interaction between the $\beta$-arrestin $\mathrm{C}$-domain and Cys-rich domains in diacylglycerol kinase (Nelson et al., 2007). Diacylglycerol kinases phosphorylate the phospholipase C (PLC) $\beta$-generated second messenger, diacylglyercol, to produce phosphatidic acid. Arrestin-dependent recruitment of diacylglycerol kinase, which inhibits protein kinase $\mathrm{C}$ (PKC) by converting diacylglycerol produced by phospholipase $\mathrm{C} \beta$ to phosphatidic acid, dampens M1 muscarinic receptormediated PKC activity.

11. Phosphatidylinositol 4-Phosphate 5-Kinase. The phosphatidylinositol 4,5-bisphosphate (PIP2)producing enzyme, phosphatidylinositol 4-phosphate 5 -kinase $\mathrm{I} \alpha$, binds $\beta$-arrestin 2 via both $\mathrm{N}$ and $\mathrm{C}$ domain interactions. Arrestin binding to phosphatidylinositol 4-phosphate 5 -kinase $\mathrm{I} \alpha$ is increased by $\beta 2$-adrenergic receptor stimulation, resulting in its recruitment to activated internalizing receptors (Nelson et al., 2008). $\beta$-Arrestin-bound phosphatidylinositol 4-phosphate 5 -kinase I $\alpha$ generates PIP2 on the inner leaflet of the clathrin-coated pit, promoting polymerization of clathrin and AP-2 and assembly of the clathrin coat. Hence, its recruitment facilitates GPCR endocytosis. Consistent with this, a $\beta$-arrestin 2 mutant deficient in PIP2 binding fails to recruit phosphatidylinositol 4 -phosphate 5-kinase I $\alpha$ or support $\beta 2$-adrenergic receptor internalization (Nelson et al., 2008).

12. Phosphatidylinositol 3-Kinase. The lipid kinase, phosphatidylinositol 3-kinase (PI3K), is an upstream regulator of protein kinase B (AKT) signaling. PI3K activity is necessary to recruit the constitutively active Ser/Thr kinase 3-phosphoinositide-dependent protein kinase-1 (PDK1) to the plasma membrane, where it phosphorylates AKT Thr ${ }^{308}$, leading to AKT activation. During protease-activated receptor (PAR)2-mediated chemotaxis, PAR2 receptors stimulate PI3K activity through a $\mathrm{G}_{\mathrm{q} / 11}-\mathrm{Ca}^{2+}$-dependent pathway, whereas $\beta$-arrestin 1 binds directly to the catalytic $\mathrm{p} 110 \alpha$ subunit of PI3K and inhibits its activity (Wang and DeFea, 2006). It has been proposed that arrestin-dependent targeting of PI3K to PAR2 receptors in pseudopodia modulates chemotaxis by locally inhibiting PI3K activity.
13. Phosphatase and Tensin Deleted on Chromosome 10. The tumor suppressor, phosphatase and tensin deleted on chromosome 10 (PTEN), regulates AKTdependent proliferative and survival signaling via both lipid phosphatase-dependent and -independent mechanisms. $\beta$-Arrestin 1 and 2 directly bind the C-terminal C2 domain of PTEN (Lima-Fernandes et al., 2011). GPCR-dependent activation of RhoA/Rho kinase signaling promotes the arrestin-PTEN association, and arrestin binding recruits PTEN to the plasma membrane and activates its lipid phosphatase activity, leading to negative regulation of AKT signaling and inhibition of cell proliferation. At the same time, $\beta$-arrestins inhibit the lipid phosphatase-independent antimigratory effects of PTEN, promoting increased cell migration.

14. Protein Phosphatase 2A-Akt-Glycogen Synthase Kinase $3 \beta$. The Ser/Thr phosphatase PP2A holoenzyme is composed of regulatory A and B subunits that target the catalytic $\mathrm{C}$ subunit to specific intracellular locations, thereby restricting its otherwise promiscuous activity to selected targets. The PP2A catalytic subunit was identified as a $\beta$-arrestin2-interacting protein in a proteomic screen (Xiao et al., 2010), and a native $\beta$-arrestin2-PP2A-AKT-glycogen synthase kinase $3 \beta$ (GSK3 $\beta$ ) complex has been purified from the striatum of mice (Beaulieu et al., 2005), suggesting that $\beta$-arrestins may serve an analogous function. In vitro, $\beta$-arrestin2, but not $\beta$-arrestin 1 , interacts directly with the $\mathrm{B}$ and $\mathrm{C}$ subunits of PP2A, AKT, and GSK3 $\beta$. In vivo, increasing synaptic dopamine release with amphetamine promotes PP2A-AKT association in wild-type, but not $\beta$-arrestin 2 knockout mice, suggesting that arrestins mediate assembly of the complex upon D2 receptor stimulation. Within the complex PP2A dephosphorylates $\mathrm{AKT} \mathrm{Thr}^{308}$, keeping its kinase activity suppressed. Because AKT phosphorylation of GSK3 $\beta$ inhibits its catalytic activity, the net result is increased GSK3 $\beta$ signaling (Beaulieu et al., 2008).

The same complex, under other circumstances, may promote AKT signaling. Angiotensin $\mathrm{AT}_{1 \mathrm{~A}}$ receptormediated, G protein-independent phosphorylation of the PP2A inhibitor, I2PP2A, transiently inhibits $\beta$-arrestin2-bound PP2A, resulting in increased AKT activity and phosphorylation-dependent inhibition of GSK3 $\beta$ (Kendall et al., 2011). Stimulation of PAR1 receptors also reportedly promotes rapid AKT activation through an unknown $\beta$-arrestin1-dependent mechanism (Goel et al., 2002). PP2A is also known to promote ERK1/2 activation by acting on c-Raf1 $\mathrm{Ser}^{259}$, an inhibitory site that must be dephosphorylated for Raf activation (Abraham et al., 2000). Because PP2A both positively regulates c-Raf (Abraham et al., 2000; Adams et al., 2005) and negatively regulates ERK1/2 (Silverstein et al., 2002; Zhou et al., 2002), its presence in GPCRarrestin complex may modulate arrestin-dependent ERK1/2 regulation. 
15. Phospholipase A2. The nicotinic acid receptor, GPR109A, recruits cytosolic phospholipase A2 (cPLA2), stimulating the release of arachidonate, the precursor of prostaglandin D2 that is the vasodilator responsible for the cutaneous flushing response seen after niacin administration. In vitro, $\beta$-arrestin 1 binds active cPLA 2 and recruits it to GPR109A (Walters et al., 2009). In $\beta$-arrestin 2 null mice, the free fatty acid-lowering effects of niacin are preserved, indicating that they are mediated via $G$ protein signaling, but the cutaneous flushing response is diminished, suggesting that arrestin-dependent recruitment of cPLA2 mediates the response.

16. Nitric Oxide Synthases. $\beta$-Arrestin2 reportedly binds and is S-nitrosylated by endothelial nitric oxide synthase (Ozawa et al., 2008). S-nitrosylation of $\beta$ arrestin 2 following $\beta 2$ adrenergic receptor activation promotes dissociation of endothelial nitric oxide synthase from the complex and facilitates arrestin binding to clathrin and AP-2, thereby accelerating receptor internalization. Although regulated transcription is the primary mechanism of inducible nitric oxide synthase (iNOS) activation, some data suggest that GPCRs can also regulate post-translational iNOS activity via an arrestin-dependent mechanism (Kuhr et al., 2010). Stimulation of bradykinin B1 receptors in lung microvascular endothelial cells promotes the association of $\beta$-arrestin 2 and iNOS and increased nitric oxide production. Conversely, the ability of $\beta$-arrestin 1 to dampen $\mathrm{NF}_{\kappa} \mathrm{B}$ signaling and repress p65/RelA transcription reportedly inhibits endoplasmic reticulum stress-induced upregulation of iNOS and moderates the inflammatory response (Tan et al., 2015).

17. Cofilin-Chronophin-LIM Kinase. GPCR-driven chemotaxis involves formation of a dominant pseudopodium at the leading edge of the cell that protrudes forward driven by F-actin polymerization and actin-myosin contraction forces. Upon activation of PAR2 receptors, $\beta$-arrestin 1 assembles a complex containing the actin filament-severing protein, cofilin, Lin11, Isl-1, and Mec-3 (LIM) kinase, and the cofilin-specific phosphatase, chronophin, that is localized to membrane protrusions. Complex assembly promotes dephosphorylation and activation of cophillin, leading to localized generation of the free barbed ends on actin filaments that permit filament extension (Zoudilova et al., 2007, 2010). Regions of both the $\mathrm{N}$ and $\mathrm{C}$ domains of $\beta$-arrestin 1 and 2 also interact with C-terminal repeat sequences within the actin-bundling protein, filamin A. Assembly of an angiotensin $\mathrm{AT}_{1 \mathrm{~A}}$ receptor- $\beta$-arrestinERK1/2-Filamin A complex is involved in the formation of membrane ruffles in Hep2 cells (Scott et al., 2006).

18. Phosphotyrosine Phosphatases. The SH2 domaincontaining protein tyrosine phosphatase (SHP)-1 modulates AKT activation by the ghrelin receptor, GHSR $1 \alpha$. In adipocytes, ghrelin activates AKT by both an early pertussis toxin-sensitive Gi/o-mediated pathway and a slower arrestin-dependent pathway (Lodeiro et al., 2011). GHSR $1 \alpha$ activation leads to c-Src activation, tyrosine phosphorylation of the p85 regulatory subunit of PI3K, PDK1 phosphorylation, and PDK1dependent activation of AKT. SHP-1 localizes to a receptor-associated arrestin-scaffold complex, where it attenuates ghrelin-induced c-Src and AKT activation. A $\beta$-arrestin2-dependent mechanism also appears to negatively regulate the activity of natural killer cells, a key component of the innate immune response. $\beta$-Arrestin2 mediates recruitment of SHP-1 and SHP2 to KIR2DL1, an inhibitory receptor of natural killer cells (Yu et al., 2008).

19. E3 Ubiquitin Ligases. Visual $/ \beta$-arrestins interact with at least five different E3 ubiquitin ligases: Mdm2, parkin, Nedd4, AIP4, and tumor necrosis factor (TNF) receptor-associated factor (TRAF)6 (Shenoy, et al., 2001, 2008; Wang et al., 2006; Bhandari et al., 2007; Ahmed et al., 2011). All four visual $/ \beta$-arrestins bind Mdm2 in cells. Unlike JNK3, which binds epitopes located in both the $\mathrm{N}$ and $\mathrm{C}$ domains of arrestin1, Mdm2 binding involves primarily the $\mathrm{N}$ domain (Song et al., 2007). In cells, Mdm2 binds with highest affinity to inactive forms of visual arrestin and $\beta$-arrestin 1 and 2 , suggesting that arrestin is preloaded with $\mathrm{Mdm} 2$ in the cytosol (Song et al., 2006). Mdm2-mediated ubiquitination of arrestins is nonetheless stimulated by receptor binding, suggesting that the conformational shifts that occur upon receptor binding promote both arrestin ubiquitination and dissociation of $\mathrm{Mdm} 2$ from the complex (Shenoy and Lefkowitz, 2005). Parkin likewise exhibits higher affinity for the cytosolic and microtubule-bound arrestin conformations than for the mutationally activated conformation (Ahmed et al., 2011). The arrestin-parkin interaction is complex, however, as it promotes Mdm2 binding while at the same time attenuating stimulus-dependent arrestin ubiquitinaiton.

Whereas $\mathrm{Mdm} 2$ catalyzes arrestin ubiquitination, other arrestin-bound E3 ligases mediate stimulusdependent ubiquitination of the receptor. The $\beta 2$ adrenergic receptor is ubiquitinated by the E3 ligase Nedd4, which is recruited by $\beta$-arrestin 2 , possibly in collaboration with ARRDC3 (Nabhan et al., 2010; Han et al., 2013). Nedd 4 promotes $\beta 2$ adrenergic receptor downregulation by accelerating its proteosomal degradation (Shenoy et al., 2008). The CXC chemokine receptor (CXCR) 4 is ubiquitinated by AIP4, which binds to the amino-terminal half of $\beta$-arrestin1 (Bhandari et al., 2007). $\beta$-Arrestin binding to another E3 ligase, TRAF6, negatively regulates TLR-interleukin (IL)-1 signaling (Wang et al., 2006). TRAF6 is normally recruited to TLR/IL-1 receptors, where it facilitates $\mathrm{I} \kappa \mathrm{B}$ kinase and $\mathrm{NF} \kappa \mathrm{B}$ activation. Binding of TRAF 6 to $\beta$-arrestin 1 and 2 in response to lipopolysaccharide or IL-1 stimulation prevents TRAF6 oligomerization and autoubiquitination, inhibiting lipopolysaccharide and IL-1 signaling. 
20. Deubiquitinases. The deubiquitinase, ubiquitinspecific protease 33 (USP33), binds both $\beta$-arrestin1 and 2 in vitro and in cells (Shenoy et al., 2009). Whereas Mdm2 catalyzes $\beta$-arrestin2 ubiquitination upon activation of $\beta 2$ adrenergic receptors, USP33 catalyzes the reverse reaction. Analysis of the kinetics of $\beta$-arrestin2 ubiquitination/deubiquitination by BRET shows that ubiquitination is detectable within 2 minutes of stimulation of either $\beta 2$ adrenergic or vasopressin V2 receptors, but within 5 minutes the $\beta 2$ receptor-bound arrestin is deubiquitinated, whereas V2 receptorbound arrestin remains stably ubiquitinated beyond 10 minutes (Perroy et al., 2004).

21. $\mathrm{Na}^{+} / \mathrm{H}^{+}$Exchanger Type 1. The $\mathrm{Na}^{+} / \mathrm{H}^{+}$exchanger regulatory factor binds to PDZ domainbinding motifs located at the very $\mathrm{C}$ terminus of several GPCRs, including the $\beta 2$ adrenergic and type $1 \mathrm{PTH}$ receptors, to regulate the activity of $\mathrm{Na}^{+} / \mathrm{H}^{+}$exchanger (NHE) type 3 controlling cell volume and $\mathrm{pH}$ (Hall et al., 1998; Mahon et al., 2002). The $\mathrm{C}$ terminus of the ubiquitous NHE1 isoform binds $\beta$-arrestin1, allowing it to be ubiquitylated by arrestin-bound Nedd4 (Simonin and Fuster, 2010). Ubiquitylation of NHE1 leads to its proteosomal degradation, and cells lacking $\beta$ arrestin 1 or Nedd4 exhibit increased plasma membrane NHE1 levels and greatly enhanced $\mathrm{Na}^{+} / \mathrm{H}^{+}$ transport actvity.

22. Regulators of Small GTPases. There are over 150 small GTPases in humans, traditionally classified into five families: Ras/Ral/Rap ( $>30$ members), Rho/Rac/Cdc42 (>20 members), Rab (>60 members), Arf (6 members), and Ran (1 member) (Takai et al., 2001). Although only one of these small GTPases has been shown to bind directly to visual $\beta$-arrestins, arrestins have been implicated in the GPCR-dependent regulation of several family members, typically through scaffolding guanine nucleotide exchange factors (GEFs), GTPase-activating proteins (GAPs), and guanine nucleotide dissociation inhibitors that regulate their activity. In so doing, visual $/ \beta$-arrestins contribute to the regulation of cell proliferation/survival, cell migration, intracellular vesicle trafficking, and cytoskeletal remodeling (Claing, 2013).

The modulation of Ras activity by $\beta$-arrestins appears to result primarily from scaffolding of Src family tyrosine kinases that function upstream of Ras (Luttrell et al., 1999) and the ERK1/2 MAPK cascade that is a major downstream Ras effector pathway (Luttrell et al., 2001). In response to $\beta 1$ adrenergic receptor activation, $\beta$-arrestin-dependent recruitment of c-Src reportedly promotes transactivation of epidermal growth factor (EGF) receptors, which in turn activate Ras by recruiting the Shc-Grb2-Sos complex (Noma et al., 2007). Conversely, data obtained using BRET biosensors suggest that in some systems the dominant role of arrestins is to dampen G proteinmediated Ras activation signals (Balla et al., 2011). In the case of Ral-GTPases, $\beta$-arrestin 1 binds directly to Ral-guanine nucleotide dissociation stimulator (GDS), a GEF for the Ras-like Ral GTPases (Bhattacharya et al., 2002). Ral-GDS binding to cytosolic $\beta$-arrestin sequesters it in the cytosol, thereby maintaining Ral in the inactive state. Recruitment of $\beta$-arrestin to activated GPCRs on the plasma membrane allows Ral-GDS to dissociate from the arrestin and activate RalA.

Although GPCR-dependent activation of Rho is mediated primarily through $\mathrm{G} \alpha_{12 / 13}$ proteins (Buhl et al., 1995), RhoA activation by the angiotensin $\mathrm{AT}_{1 \mathrm{~A}}$ receptor is inhibited by knockdown of $\beta$-arrestin 1 and can be stimulated by an arrestin pathway-biased angiotensin analog (Barnes et al., 2005). $\beta$-Arrestin1 is involved in RhoA-mediated actin stress fiber formation and membrane blebbing. One mechanism by which arrestin-dependent RhoA regulation may occur is through direct binding of ARFGAP21, a RhoA GAP (Anthony et al., 2011). Stimulation of angiotensin $\mathrm{AT}_{1 \mathrm{~A}}$ receptors promotes the $\beta$-arrestin1-ARFGAP21 interaction, occluding its GAP domain and leading to increased RhoA activity and membrane ruffling. Notably, RhoA activation potentiates the interaction between $\beta$-arrestin1 and PTEN, whereas knockdown of $\beta$-arrestin $1 / 2$ inhibits RhoA-dependent PTEN activation by the lysophosphatidic acid (LPA) receptor, suggesting bidirectional crosstalk between arrestin and RhoA signaling (Lima-Fernandes et al., 2011). $\beta$ Arrestins have been reported to play both positive and negative roles in Rac1 signaling. Arrestins inhibit the NAPDH oxidase-dependent oxidative cell burst produced by IL-8 and protect against cell death, while at the same time mediating Rac1 activation by Wnt-5A (Zhao et al., 2004; Bryja, et al., 2008). In HEK293 cells, $\beta 2$ adrenergic receptor-dependent activation of Rac1 is blocked by knockdown of $\beta$-arrestin1, as is Rac1dependent activation of NADPH oxidase and p38 MAPK (Gong et al., 2008). $\beta$-Arrestins negatively regulate PAR2 receptor-mediated Cdc42 activation through an undefined mechamism (Wang et al., 2007). Similarly, in breast and ovarian cancer cell lines, activation of Cdc42 by the type III transforming growth factor- $\beta$ receptor, a non-GPCR tumor suppressor, alters actin cytoskeletal rearrangement and reduces random cell migration (Finger et al., 2008; Mythreye and Blobe, 2009).

Rab family GTPases control most aspects of vesicular trafficking, and Rab4, Rab5, Rab7, and Rab11 are involved in GPCR endocytosis, recycling, and lysosomal targeting (Seachrist and Ferguson, 2003). Although the stability of the GCPR-arrestin complex has a profound impact on intracellular trafficking, there are no data to indicate that arrestins directly bind either Rabs or their GEFs and GAPs. In contrast, ARF6, a small GTPase involved in sequestration of many GPCRs, binds directly to the $\mathrm{C}$-terminal domain of $\beta$-arrestin 1 and 2 in 
the GDP-bound state, where it is activated by ARNO, a constitutively arrestin-associated ARF-GEF (Claing et al., 2001; Houndolo, et al., 2005; Macia et al., 2012). Activated ARF6 mediates the recruitment of clathrin and AP-2 to the GPCR-arrestin complex, nucleating the assembly of endocytic vesicles (Paleotti et al., 2005; Poupart et al., 2007). The association between ARNO and $\beta$-arrestin also facilitates GPCR binding and desensitization, as shown for the luteinizing hormone (LH) receptor (Mukherjee et al., 2000).

23. N-Ethylmaleimide-Sensitive Fusion Protein. $\mathrm{N}$-ethylmaleimide-sensitive fusion protein (NSF) is a homohexameric ATPase that regulates the disassembly of soluble NSF attachment protein (SNAP) receptor (SNARE) complexes that facilitate the membrane fusion events necessary for vesicle transport (Rizo and Südhof, 2002). Full-length $\beta$-arrestin1 preferentially binds to the N-terminal SNAP/SNARE binding domain of NSF when ATP-bound (McDonald et al., 1999). Overexpression of NSF facilitates $\beta 2$ adrenergic receptor endocytosis, suggesting that arrestin-dependent NSF recruitment contributes to clathrin-dependent GPCR internalization. Similarly, visual arrestin binds to NSF and stimulates its activity, enhancing neurotransmitter secretion in rod photoreceptors (Huang et al., 2010).

24. Wnt Signaling Pathway-Dishevelled. Wnts are secreted glycoproteins involved in embryologic patterning and development. They bind to seven-membranespanning receptors called Frizzleds, which cluster within the GPCR superfamily but do not signal via heterotrimeric G proteins. Frizzleds $(\mathrm{Fz})$ recruit cytosolic proteins called Dishevelleds (Dsh), and the Fz-Dsh complex mediates the endocytosis and degradation of Wnt protein, a key step in establishing morphogen gradients during development (Dubois et al., 2001). During canonical Wnt signaling, Wnts bind to $\mathrm{Fz}$, activating Dsh, and preventing Axin/GSK3 $\beta$-mediated phosphorylation and degradation of $\beta$-catenin. The resulting nuclear accumulation of $\beta$-catenin leads to activation of $\mathrm{T}$ cell-specific factor/lymphoid enhancer factor (LEF) transcription factors. In the alternative noncanonical Wnt/planar cell polarity signaling pathway, Wnts signal through Fz to the small GTPases Rho and Rac to promote rearrangement of the actin cytoskeleton. Another noncanonical Wnt pathway promotes increases in intracellular $\mathrm{Ca}^{2+}$ to negatively regulate the canonical $\mathrm{Wnt} / \beta$-catenin pathway. $\beta$ Arrestin1 binds phosphorylated Dsh1 and Dsh2 and enhances LEF-mediated transcription (Chen et al., 2001). $\beta$-Arrestin2 interacts with both Axin and Dsh after Wnt3A stimulation (Bryja et al., 2007), suggesting both $\beta$-arrestins are involved in negatively regulating GSK3 $\beta$ activity and promoting canonical Wnt signaling. During noncanonical wnt5A signaling, $\beta$-arrestin2 binds phosphorylated Dsh2, and, in a heterologous expression system, Wnt5A-stimulated endocytosis of
Fz4 is dependent upon both $\beta$-arrestin 1 and Dvl2 (Chen et al., 2003).

25. Hedgehog Signaling Pathway-Smoothened. The Hedgehog signaling pathway regulates cell fate determination during embryologic patterning. Smoothened, a non-G protein-coupled seven-membranespanning receptor, is constitutively suppressed by binding to Patched, a 12-membrane-spanning coreceptor that binds the extracellular glycoprotein, Sonic hedgehog (Shh). Shh binding to Patched relieves its inhibition of Smoothened, which in turn activates Gli family transcription factors by dissociating them from their negative regulator, $\mathrm{Su}(\mathrm{fu}) . \beta$-Arrestin 2 binds activated Smoothened in a GRK2-dependent manner and promotes its internalization (Chen et al., 2004). Arrestins also promote the association of Smoothened with the kinesin motor protein, Kif3A, causing $\beta$-arrestin, Smoothened, and Kif3A to colocalize in primary cilia (Kovacs et al., 2008). In NIH3T3 cells, downregulation of $\beta$-arrestin 1 or 2 causes mislocalization of Smoothened and disrupts activation of Gli1.

26. Nuclear Proteins and Transcription Factors. By virtue of its NLS, $\beta$-arrestin 1 is able to participate in protein-protein interactions within the nucleus that modify the activity of several transcription factors. Besides binding nuclear p65/RelA and enhancing $\mathrm{NF}_{\kappa} \mathrm{B}$ signaling by the bradykinin receptor, activation of $\delta$-opioid receptors causes $\beta$-arrestin 1 to move into the nucleus, where it interacts with the p27 and c-Fos promoters and stimulates transcription by recruiting histone acetyltransferase p300 and enhancing local histone H4 acetylation (Kang et al., 2005). Nuclear $\beta$-arrestin 1 also binds enhancer of zeste homolog 2 , a polycomb group ( $\mathrm{PcG}$ ) protein involved in gene silencing (Qin et al., 2014). Knockdown of $\beta$-arrestin 1 results in reduced histone $\mathrm{H} 4$ acetylation of many genes in K562 chronic myelogenous leukemia (CML) cells, notably including the $\mathrm{B}$ cell receptor/Abelson murine leukemia viral oncogene homolog 1 (ABL) fusion oncogene, leading to reduced B cell receptor/ABL expression. Importantly, depletion of $\beta$-arrestin 1 slows proliferation of K562 and primary CML cells and increases survival of CML mice. In zebrafish, $\beta$-arrestin 1 binds the PcG recruiter, YY1, a ubiquitously expressed transcription factor essential for embryonic development (Yue et al., 2009). $\beta$-Arrestin1 binding sequesters YY1, relieving PcG-mediated repression of $\mathrm{Cdx} 4$-hox transcription. Without $\beta$-arrestin 1 , hox gene expression is downregulated, leading to developmental defects and failed hematopoiesis.

Within the nucleus, $\beta$-arrestin 1 interacts directly with the peroxisome proliferator-activated receptor (PPAR) $\gamma$ via a short region in the C-terminal domain between $\mathrm{M}^{255}$ and $\mathrm{A}^{263}$ (Zhuang et al., 2011). $\beta$-Arrestin competes with the 9 -cis retinoic acid receptor $(\mathrm{RXR}) \alpha$, inhibiting PPAR $\gamma-\mathrm{RXR} \alpha$-dependent transcription and promoting PPAR $\gamma$ nuclear receptor corepressor 
function. As a result, loss of $\beta$-arrestin 1 promotes, and overexpression of $\beta$-arrestin 1 inhibits, adipogenesis, macrophage infiltration, and diet-induced obesity, and improves glucose tolerance and systemic insulin sensitivity in vivo. The $\beta$-arrestin $1 \mathrm{C}$-terminal domain also directly interacts with signal transducers and activators of transcription (STAT) 1 and its regulatory phosphatase TC45 in the cell nucleus following STAT1 activation by interferon- $\gamma$ (Mo et al., 2008). By acting as a scaffold for STAT1 dephosphorylation by the nuclear phosphatase TC45, $\beta$-arrestin 1 negatively regulates interferon- $\gamma$ signaling and cellular antiviral responses.

In contrast to $\beta$-arrestin1, $\beta$-arrestin2 contains a classic leucine-rich nuclear export sequence (NES) located between amino acid residues 390-400 of the C-terminal domain (Scott et al., 2002). As a result, $\beta$-arrestin2 is actively excluded from the nucleus. Interestingly, $\beta$-arrestin 2 actively accumulates in the nucleus when the NES is mutated to the corresponding residues of $\beta$-arrestin 1 or when nuclear export is pharmacologically inhibited by leptomycin B, suggesting that it may also engage in nucleocytoplasmic shuttling.

\section{B. Functional Pools of Arrestin}

The visual $/ \beta$-arrestins possess three properties essential to their scaffolding function: the flexibility to bind multiple cargo proteins; the capacity to exist in different intracellular pools wherein they adopt different conformations; and the ability to recognize activated GPCRs. Whereas many arrestin cargo proteins appear to be constitutively associated, others exhibit a distinct preference for the cytosolic, microtubule-bound, or GPCR-bound arrestin. Thus, arrestin binding can constrain signaling proteins to one cellular compartment until an external GPCR-mediated stimulus prompts a conformational change that causes them to release some cargos and associate with others (Fig. 4).

1. Cytosolic Arrestin. In the absence of an acute stimulus, most of the visual $/ \beta$-arrestin pool resides either in the cytosol in an inactive conformation or bound to microtubules. Several cargos have been shown to have higher affinity for inactive arrestin than for either the microtubule- or GPCR-bound conformations. The binding site for $\mathrm{Ca}^{2+}$-calmodulin overlaps the GPCR and microtubule-binding surfaces such that it can only interact with free arrestin protein (Wu et al., 2006). Binding to cytosolic arrestins probably serves to buffer intracellular $\mathrm{Ca}^{2+}$-calmodulin and to maintain an equilibrium between cytosolic and microtubule-bound arrestin in the absence of higher-affinity GPCR docking sites. JNK3 also exhibits higher affinity for the cytosolic form of $\beta$-arrestin2 (Song et al., 2006; Breitman et al., 2012 ). Because the NES of $\beta$-arrestin 2 keeps it out of the nucleus, arrestin-bound JNK3 is maintained in the cytosol away from its nuclear transcription factor targets. In the case of $\beta$-arrestin1, which freely enters the nucleus in its monomeric form, IP6 binding promotes arrestin self-association and hinders spontaneous nuclear translocation (Milano et al., 2006; Song et al., 2006, 2007). Another cargo that prefers inactive arrestin is Mdm2 (Song et al., 2006). In this case, the differential affinity probably permits dynamic regulation, in that $\mathrm{Mdm} 2$ preferentially ubiquitinates GPCR-bound arrestin, a step that stabilizes the GPCRarrestin complex (Shenoy and Lefkowitz, 2005). Once the arrestin is in the ubiquitinated GPCR-bound conformation, the drop in affinity may allow Mdm2 to dissociate, allowing other cargos to take its place in the receptorassociated complex.

2. Microtubule-Bound Arrestin. Like $\mathrm{Ca}^{2+}$-calmodulin, the microtubule binding sites on arrestins overlap the GPCR interacting sites such that microtubule and GPCR binding are mutually exclusive (Nair et al., 2004; Hanson et al., 2006b). Also like $\mathrm{Ca}^{2+}$-calmodulin, the affinity of arrestins for microtubules is much lower than for activated GPCRs. Thus, competition between abundant low-affinity microtubule binding sites and a smaller number of ligand-induced high-affinity GPCR binding sites probably allows arrestin to sequester itself, and certain cargos, in a microtubule-associated pool under basal conditions. All visual $/ \beta$-arrestins can bind microtubules, although $\beta$-arrestins have somewhat higher affinity (Hanson et al., 2007a). Notably, deletions in the hinge region of $\beta$-arrestin 1 that restrict its flexibility enhance microtubule binding compared with either the wild-type protein or preactivated mutants that show enhanced receptor binding. This suggests the GPCR- and microtubule-bound conformations both differ from that of free cytosolic arrestin. Because the binding of some arrestin cargos is sensitive to arrestin conformation, it is thus likely that cytosolic, microtubule-bound, and GPCR-bound arrestins carry different cargos. For example, ERK1/2 has significant affinity only for the GPCR- and microtubule-bound arrestin conformations, but is activated only upon recruitment to the receptor. Sequestration of inactive ERK1/2 within the microtubule-bound arrestin pool effectively dampens basal ERK1/2 activity, thereby enhancing the signal-to-noise ratio upon receptor activation (Hanson et al., 2007a; Coffa et al., 2011b). Conversely, Mdm2 loses affinity for the GPCR-bound arrestin conformation, such that overexpressing arrestins markedly increases ubiquitination of microtubuleassociated substrates (Hanson, et al., 2007a). These conformational effects are cargo-specific, in that other arrestin cargos, e.g., JNK3 and PP2A, are not preferentially targeted to microtubules.

The capacity of $\beta$-arrestins to bind both microtubules and clathrin enables them to regulate focal adhesion dynamics (Hanson et al., 2007a; Cleghorn et al., 2015). $\beta$-Arrestin1/2 null murine fibroblasts exhibit increased cell spreading and adhesion, reduced motility, and 


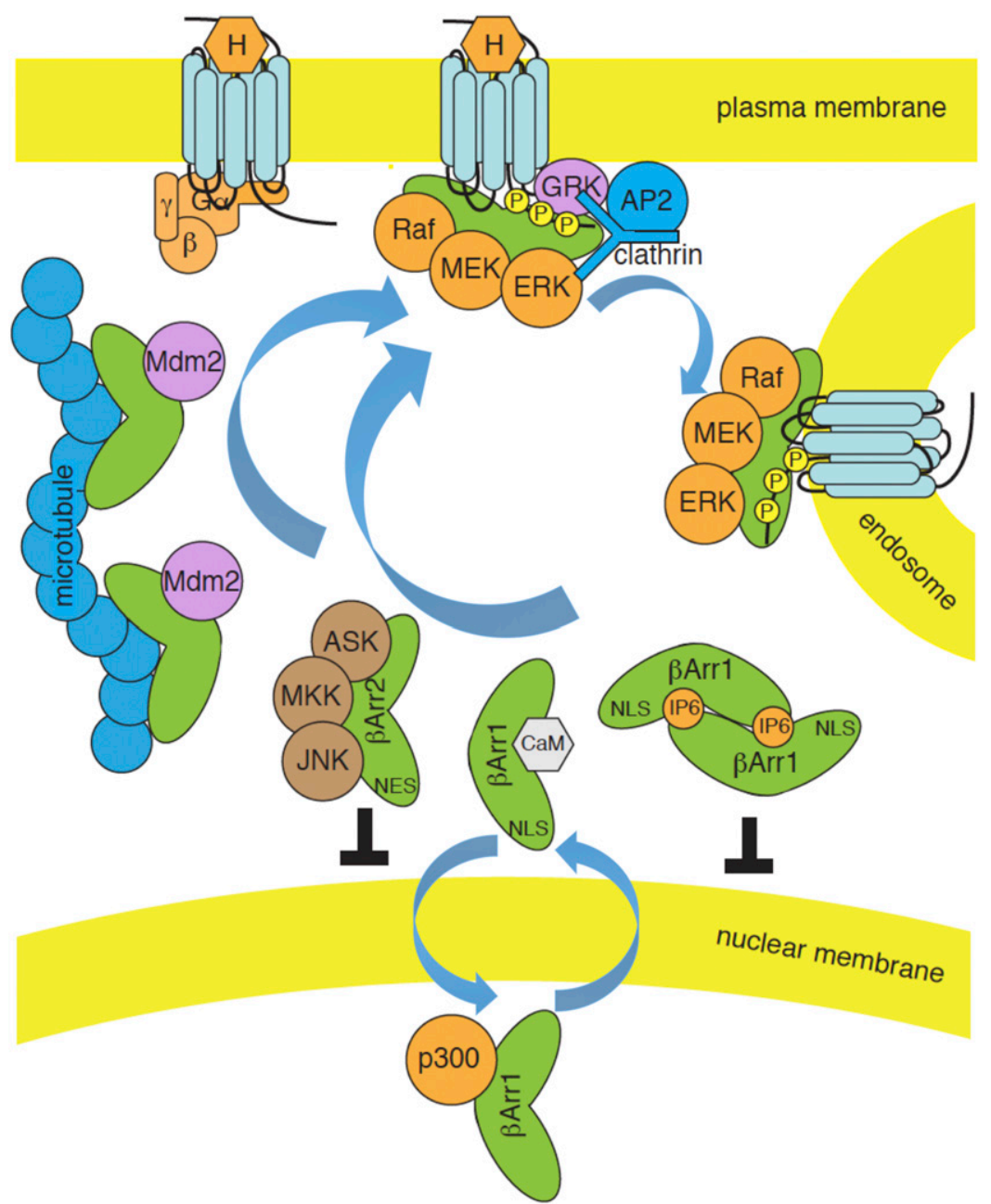

Fig. 4. Dynamic regulation of functionally discrete arrestin pools. Visual/ $\beta$-arrestins exist in equilibrium between a large intracellular pool, where they are either freely cytosolic or associated with low-affinity microtubule binding sites, and a small pool bound with high affinity to activated GPCRs. Cytosolic, microtubule-bound, and GPCR-bound arrestins adopt different conformations, such that some cargos preferentially associate with free arrestins, e.g., $\mathrm{Ca}^{2+}$-calmodulin and components of the ASK1/MKK4/JNK3 cascade, others prefer microtubule-bound arrestin, e.g., Mdm2, whereas still others preferentially associate with GPCR-bound arrestin, e.g., Raf-MEK-ERK1/2. Upon ligand (H) binding, GRK-phosphorylated GPCRs recruit $\beta$-arrestins from the cytosolic and microtubule-bound pools to the plasma membrane, where they can engage clathrin and AP2, leading to receptor endocytosis. Assembly of multiprotein signaling complexes on the GPCR-arrestin scaffold leads to spatially contrained pools of activated cargo, e.g., ERK1/2. Although $\beta$-arrestin2 ( $\beta$ Arr2) is excluded from the cell nucleus by its NES, $\beta$-arrestin1 is in equilibrium between cytosolic and nuclear pools. IP6 binding promotes $\beta$-arrestin1 self-association, which, like microtubule binding, sequesters it from the nucleus and restrains interactions with transcriptional regulatory proteins, e.g., histone acetyltransferase p300.

reduced focal adhesion turnover. Reintroduction of wild-type $\beta$-arrestin 1 and 2 , or mutants defective in GPCR binding, can each restore focal adhesion dynamics, whereas mutants defective in clathrin binding cannot. Because other arrestin cargos, e.g., c-Src, ERK1/2, and JNK, are known regulators of focal adhesion assembly, it is likely that $\beta$-arrestin 1 and 2 regulate cell adhesion by bringing clathrin and possibly other effectors to microtubules in a GPCRindependent manner.

3. GPCR-Bound Arrestin. The unique capacity of visual $/ \beta$-arrestins to respond to extracellular stimuli derives from their ability to recognize and bind agonistoccupied GRK phosphorylated GPCRs on the plasma membrane. Heptahelical GPCRs function by detecting the presence of extracellular ligands that, upon interacting with the receptor, promote conformational rearrangements, which are in turn transmitted across the plasma membrane to affect the conformation and activity of intracellular effectors, such as heterotrimeric G proteins and arrestins (Kenakin, 2012; Manglik and Kobilka, 2014). The conformational shifts occurring in visual $/ \beta$-arrestins not only permit tight binding to receptors, but also affect their affinity for some cargos. Destabilization of the arrestin polar core upon interaction with receptor-attached phosphates exposes the LIEF and RxR motifs in the $C$ terminus of $\beta$-arrestin $1 / 2$, permitting them to engage clathrin and AP-2, steps that are essential for clathrin-dependent endocytosis of GPCRs (Goodman et al., 1997; Laporte et al., 2000). The binding of some signaling cargos is likewise affected. cRaf-1 and activated ERK1/2 have highest affinity for the receptor-bound arrestin conformation, allowing the GPCR-arrestin complex to nucleate 
assembly of a signalsome that not only activates ERK1/2, but also keeps it spatially constrained (Luttrell et al., 2001; Coffa et al., 2011b). Receptorbound arrestins also direct assembly of the cofilinchronophin-LIM kinase complex necessary for actin cytoskeletal rearrangement and chemotactic cell migration (Zoudilova et al., 2007, 2010). Other cargos, for example Ral-GDS (Bhattacharya et al., 2002) and Mdm2 (Song et al., 2006), lose affinity for GPCRbound arrestin, such that they are released upon receptor binding, freeing them to engage other membrane-associated substrates and making room for new signalsome components. This potential for dynamic regulation of GPCR-bound arrestin signalsomes is highlighted by biophysical studies of the effects of receptor binding on arrestin conformation, which indicate that differences in GPCR intracellular domain structure stabilize different $\beta$-arrestin 2 conformations (Lee et al., 2016; Nuber et al., 2016). To the extent that the affinity of at least some arrestin cargos is sensitive to arrestin conformation, this implies that GPCRs may specify which arrestin effectors can bind.

The stability of the GPCR-arrestin complex also impacts the kinetics of arrestin-dependent signaling. For example, GPCRs that form stable arrestin complexes, like the angiotensin $\mathrm{AT}_{1 \mathrm{~A}}$ and vasopressin $\mathrm{V}_{2}$ receptors (Oakley et al., 2000), remain bound to activated ERK1/2, leading both to prolonged ERK1/2 activation and targeting of the kinase to endosomes (DeFea et al., 2000b; Luttrell et al., 2001; Ahn et al., 2004; Jafri et al., 2006). These constraints have profound effects on ERK1/2 function. Whereas ERK1/2 activated by classic growth factors or $\mathrm{G}$ proteindependent GPCR signaling is able to translocate to the nucleus and elicit a transcriptional response, ERK1/2 bound to $\beta$-arrestin scaffolds is retained in the cytosol and silent in Elk-1 reporter assays (Tohgo et al., 2002; Lee et al., 2008). Arrestin-bound ERK1/2 performs other functions, for example, regulating arrestinclathrin interaction during GPCR endocytosis (Lin et al., 1999), modulating actin cytoskeletal reorganization during chemotaxis (Ge et al., 2003), and activating MNK1, p90RSK, and p70S6K signaling to stimulate protein translation (DeWire et al., 2008; Kendall et al., 2014). Thus, by compartmentalizing signaling, arrestin scaffolding can change the functional consequences of pathway activation, even when the pathway is subject to convergent regulation by multiple mechanisms.

4. Nuclear Arrestin. With the exception of $\beta$-arrestin2, all visual $/ \beta$-arrestins are able to enter the nucleus. The presence of a NES in $\beta$-arrestin2 (Scott et al., 2002) largely confines it to cytosolic, microtubule-bound, and GPCR-bound pools, and favors cytosolic sequestration of cargos that regulate nuclear substrates, e.g., JNK3 (Song et al., 2006). In contrast, $\beta$-arrestin1, by virtue of its intrinsic NLS, appears capable of regulating events within the nucleus. At concentrations present in the cytosol, IP6 promotes $\beta$-arrestin1 self-assembly and retards its nuclear translocation (Milano et al., 2006; Hanson et al., 2007a). Because the low-affinity IP6 interaction must be displaced for GPCR binding to occur, receptor activation might be expected to disassemble $\beta$-arrestin 1 oligomers and promote nuclear signaling through engagement of components of the $\mathrm{NK}_{\kappa} \mathrm{B} / \mathrm{p} 65 / \mathrm{RelA}$ pathway (Hoeppner et al., 2012), STAT1/TC45 (Mo et al., 2008), p300 histone acetyltransferase (Kang et al., 2005), and other regulators of transcription. The role of IP6 in regulating nuclear arrestin signaling is likely to be complex, however, because IP6 inhibits, rather than enhances, self-association of visual arrestin (Hanson et al., 2007a).

\section{Arrestins as GPCR-Activated Scaffolds}

Nearly all GCPRs function as GEFs for heterotrimeric G proteins. Agonist binding stabilizes receptor conformations that enable it to catalyze GTP for GDP exchange on heterotrimeric $\mathrm{G}$ protein $\mathrm{G} \alpha$ subunits, leading to dissociation of GTP-bound $\mathrm{G} \alpha$ and $\mathrm{G} \beta \gamma$ subunits, which in turn regulate the activity of enzymatic effectors, such as adenylate cyclases, PLC isoforms, and ion channels, and generate small-molecule second messengers that control the activity of key enzymes involved in intermediary metabolism. What then are the principal roles of arrestin scaffolds in cells? For the most part, arrestin-mediated signals appear to coordinate a few basic biologic processes, some related to modulation of $\mathrm{G}$ protein signaling and others accomplished by conferring upon GPCRs the ability to regulate noncanonical GPCR signaling pathways (Fig. 5).

1. Negative Regulation of Heterotrimeric G Protein Signaling. The most conserved arrestin function is the negative regulation of $G$ protein signaling through direct steric hindrance of the GPCR-G protein interaction, and in the case of $\beta$-arrestins mediation of clathrindependent receptor sequestration (Ferguson, 2001). Due to common sites of interaction, GPCR binding to arrestins and G proteins is mutually exclusive (Kang et al., 2015). Even the formation of megaplexes, in which $\beta$-arrestins adopt a partially engaged binding pose that permits simultaneous binding of arrestin and $\mathrm{G}$ protein (Thomsen et al., 2016), appears to support receptor endocytosis, removing receptors from the plasma membrane even while permitting ongoing $\mathrm{G}$ protein signaling (Calebiro et al., 2009; Ferrandon et al., 2009; Mullershausen et al., 2009; Feinstein et al., 2013; Vilardaga et al., 2014).

Besides serving as adaptors linking GPCRs to clathrin and AP-2, $\beta$-arrestins also carry cargos that either promote second-messenger degradation or modulate the endocytic process. Gs-coupled $\beta 2$ adrenergic receptors form a complex with $\beta$-arrestin2 and PDE4D3/5, leading to accelerated cAMP degradation (Perry et al., 2002). Whereas wild-type $\beta$-arrestin 2 is able to rescue arrestin-dependent inhibition of $\beta 2$-adrenergic 


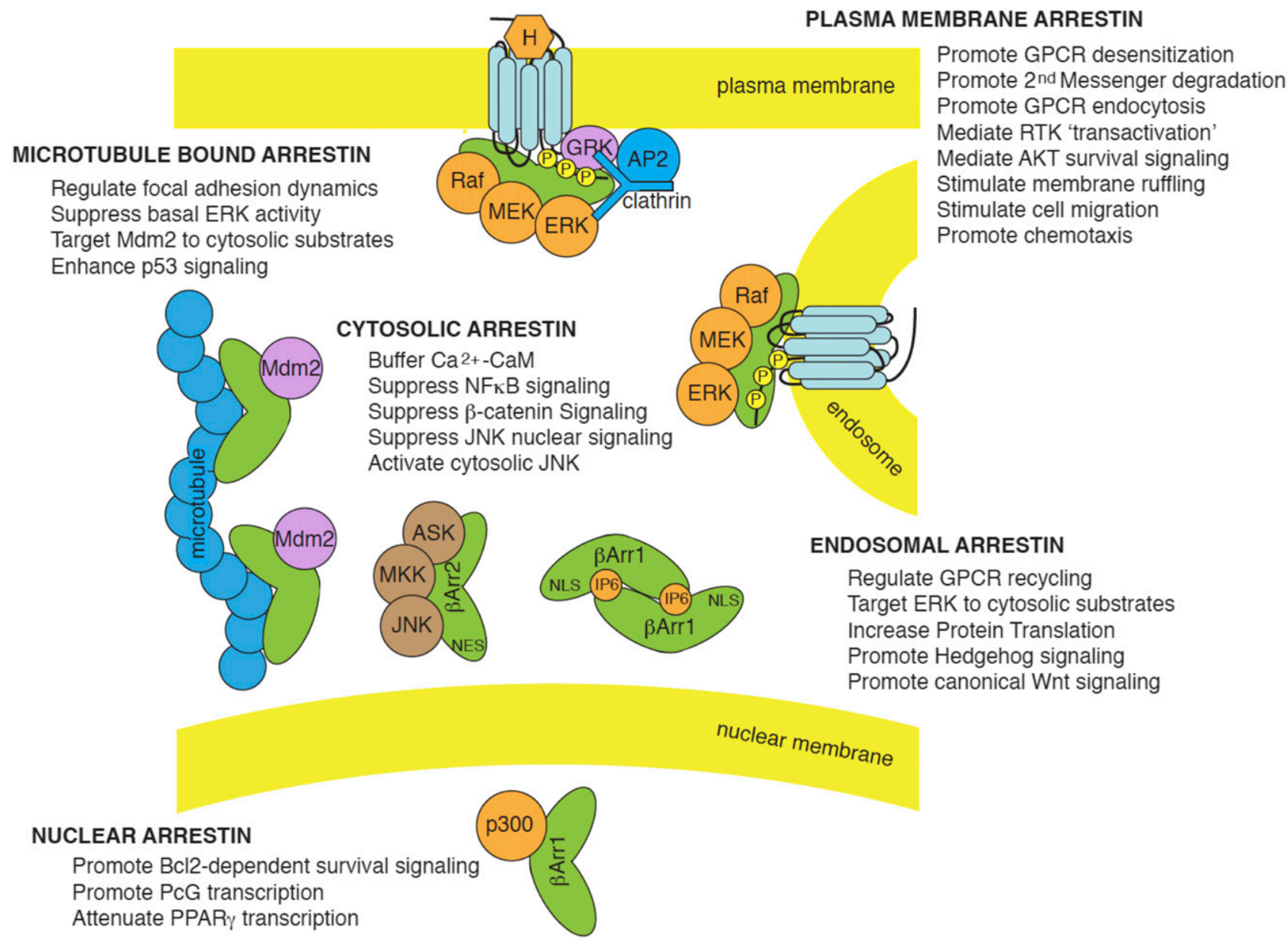

Fig. 5. Diverse cellular functions of arrestin scaffolds. By associating with different cargos in different subcellular locations, visual $/ \beta$-arrestins regulate multiple signaling networks. In quiescent cells, free cytosolic and microtubule-bound arrestins dampen basal pathway activity by sequestering signaling pathway intermediates away from their site of activation/action. Free arrestins can buffer cytosolic $\mathrm{Ca}^{2+}-\mathrm{CaM}$ concentration, suppress $\mathrm{NF}_{\kappa} \mathrm{B}$ signaling by sequestering $\mathrm{I}_{\kappa} \mathrm{B}$ kinases, and tonically inhibit $\beta$-catenin signaling by promoting GSK3 $\beta$-dependent $\beta$-catenin phosphorylation and degradation. They also keep proapoptotic JNK kinases away from their nuclear substrates, whereas, in the case of $\beta$-arrestin2, they promote activation of cytosolic JNK. Microtubule-bound arrestins sequester inactive ERK1/2 away from the plasma membrane, dampening basal pathway activity, while directing Mdm2 toward cytoskeletal substrates. In some settings, this has the effect of increasing proapoptotic p53 signaling by preventing p53 ubiquitination and degradation. Microtubule-bound arrestins also regulate cell adhesion by binding to regulators of focal adhesions such as Src, ERK1/2, and JNK. Once recruited to plasma membrane-bound GPCRs, arrestins promote GPCR desensitization, support clathrin-dependent endocytosis, and accelerate second-messenger degradation by recruiting cAMP phosphodiesterases and diacylglycerol kinase. At the membrane they also stimulate cell proliferation by promoting Src-dependent transactivation of EGF receptor tyrosine kinases (RTKs) and promote cell survival by activating AKT. Through their interactions with numerous regulators of actin cytoskeletal dynamics, arrestin stimulate membrane ruffling, cell migration, and chemotaxis. Several $\beta$-arrestin cargos, e.g., ERK1/2, continue to signal from endosomal GPCR-arrestin signalsome complexes, where they regulate aspects of GPCR trafficking and recycling and preferentially phosphorylate cytosolic ERK substrates, leading to increased protein translation. $\beta$-Arrestins also stimulate canonical Wnt signaling by engaging Dsh and inhibiting GSK3 $\beta$ to stabilize $\beta$-catenin, and promote hedgehog signaling by internalizing and targeting smoothened to primary cilia. Within the nucleus, $\beta$-arrestin 1 interacts with a number of transcription factors to either increase or tonically inhibit transcription.

receptor-stimulated protein kinase $\mathrm{A}(\mathrm{PKA})$ activation in $\beta$-arrestin $1 / 2$ null fibroblasts, $\mathrm{R}^{26} \mathrm{~A}$ and $\mathrm{R}^{286} \mathrm{~A} \mathrm{mu}-$ tants of $\beta$-arrestin2 that lack PDE4D5 binding but retain the ability to bind activated GPCRs are much less effective terminators of cAMP-PKA signaling (Baillie et al., 2007). In an analogous manner, arrestindependent recruitment of diacylglycerol kinase, which inhibits PKC by converting diacylglycerol produced by $\operatorname{PLC} \beta$ to phosphatidic acid, dampens Gq/11-mediated signaling by the M1 muscarinic receptor (Nelson et al., 2007). It remains unclear whether or how specificity is achieved in arrestin-dependent targeting of PDE4D3/5 and diacylglycerol kinase, e.g., whether activation of adenylyl cyclase or PLC generates a coregulatory signal that directs these second-messenger degrading enzymes to the appropriate receptor. The original reports suggest that their interaction with $\beta$-arrestins is constitutive; however, the effect of arrestin conformation on binding of these cargos has not been determined.

The reversible ubiquitination of arrestin3 regulates the stability of the GPCR-arrestin complex and thereby the kinetics of receptor desensitization, internalization, and intracellular trafficking. Ubiquitination of $\beta$-arrestin2 by Mdm2 stabilizes the receptor-arrestin interaction, allowing the receptor to undergo endocytosis, after which USP33 removes the ubiquitin moiety, promoting arrestin dissociation (Shenoy and Lefkowitz, 
2003; Shenoy et al., 2009). This allows class A GPCRs, like the $\beta 2$ adrenergic receptor, to recycle to the plasma membrane. In contrast, $\beta$-arrestin recruited to class $\mathrm{B}$ receptors, like the angiotensin $\mathrm{AT}_{1 \mathrm{~A}}$ receptor, remains ubiquitinated, stabilizing the receptor-arrestin complex and favoring slow recycling or receptor degradation. Hence, a lysine-less $\beta$-arrestin 2 mutant is unable to remain associated with the $\beta 2$ adrenergic receptor (Shenoy et al., 2007); expression of a $\beta$-arrestin2-ubiquitin chimera converts the $\beta 2$ adrenergic receptor from class A to class B trafficking (Shenoy and Lefkowitz, 2003); and expression of a $\beta$-arrestin $2 \mathrm{~K}^{11 / 12} \mathrm{R} \mathrm{mu}$ tant that cannot be ubiquitinated upon binding to the angiotensin $\mathrm{AT}_{1 \mathrm{~A}}$ receptor promotes class $\mathrm{A}$ trafficking (Shenoy and Lefkowitz 2005). By stabilizing the receptor-arrestin interaction, ubiquitination of $\beta$-arrestin2 also favors ERK1/2 activation in GPCRbased signalsomes (Shenoy et al., 2007). As mentioned, ubiquitination of the receptor itself by other $\beta$-arrestinbound ubiquitin ligases, e.g., Nedd4 and AIP4, influences GPCR fate by accelerating their degradation in proteosomes (Bhandari et al., 2007; Shenoy et al., 2008).

Arrestin-bound kinases and phosphatases also modulate receptor endocytosis and trafficking. Arrestinscaffolded c-Src phosphorylates GRK2, providing negative feedback on receptor desensitization by destabilizing GRK2 and promoting its rapid proteosomal degradation (Penela et al., 2001). Arrestin-Src binding promotes phosphorylation of $\mathrm{Tyr}^{497}$ of dynamin1, which regulates dynamin self-assembly (Ahn et al., 1999, 2002). The $\beta 2$ adaptin subunit of AP-2 is also subect to regulation by arrestin-dependent Src phosphorylation (Fessart et al., 2005, (2007; Zimmerman et al., 2009). c-Src stabilizes the association of $\beta$-arrestin2 and $\beta 2$ adaptin independent of its kinase activity. Srcmediated phosphorylation of $\beta 2$ adaptin $\mathrm{Tyr}^{737}$ in clathrin-coated pits leads to dissociation of AP-2 from the complex, permitting the receptor-arrestin complex to exit the clathrin-coated vesicle.

The fact that active ERK1/2 only has high affinity for receptor-bound arrestins enables class B GPCRs to generate a spatially constrained pool of ERK1/2 that localizes to endosomes (DeFea et al., 2000b; Tohgo et al., 2002, 2003; Ahn et al., 2004; Lee et al., 2008). As a result, arrestin-dependent ERK1/2 catalytic activity appears to be directed toward membrane or cytosolic substrates. ERK1/2 phosphorylates $\mathrm{Ser}^{412}$ in the C terminus of $\beta$-arrestin 1 , limiting its ability to bind clathrin (Lin et al., 1999). $\beta$-arrestin1 in the cytosol is almost stoichiometrically phosphorylated on $\mathrm{Ser}^{412}$, and dephosphorylation of $\mathrm{Ser}^{412}$ upon receptor binding promotes receptor internalization and ERK1/2 activation. Rephosphorylation by ERK1/2 in the signalsome complex probably either provides negative feedback regulation of receptor endocytosis or facilitates receptor internalization by promoting dissociation of $\beta$-arrestin 1 and clathrin, allowing the receptor to exit clathrin- coated vesicles. In rodent, but not human, $\beta$-arrestin2, ERK1/2 phosphorylation of $\mathrm{S}^{178}$ stabilizes the GPCRarrestin complex in endosomes and delays recycling (Khoury et al., 2014). Because casein kinase II has been implicated in phosphorylation of $\mathrm{Thr}^{383}$ of $\beta$-arrestin2, which destabilizes the interaction between arrestin and $\beta 2$ adrenergic receptors (Lin et al., 2002), it is possible that arrestin recrutiment of casein kinase II plays a role similar to that proposed for ERK1/2-mediated phosphorylation of $\beta$-arrestin $1 \mathrm{Ser}^{412}$ (Lin et al., 1997).

Arrestin-bound PP2A may also contribute to GPCR trafficking and resensitization. $\beta$-Arrestin1-bound PP2A reportedly dephosphorylates $\beta$-arrestin $1 \operatorname{Ser}^{412}$, a step that regulates the interaction between arrestin and the clathrin-coated pit (Hupfeld et al., 2005). Dephosphorylation of GRK-phosphorylated receptors, a prerequisite for receptor resensitization, also involves PP2A. A 150-kDa oligomeric form of PP2A catalyzes the dephosphorylation of $\beta 2$ and $\alpha 2$ adrenergic receptors within the acidic microenvironment of endosomes, allowing receptors to recycle to the plasma membrane (Pitcher et al., 1995; Krueger et al., 1997). Arrestinbound modulators of cytoskeletal dynamics also play a role. Activated ARF6 mediates the recruitment of clathrin and AP-2 to the GPCR-arrestin complex, nucleating the assembly of endocytic vesicles (Paleotti et al., 2005; Poupart et al., 2007). The association between ARNO and $\beta$-arrestin also facilitates GPCR binding and desensitization, as shown for the $\mathrm{LH}$ receptor (Mukherjee et al., 2000). $\beta$-arrestin1 binding to the SNAP/SNARE binding domain of ATP-bound NSF facilitates the membrane fusion events necessary for vesicle transport, and overexpression of NSF facilitates $\beta 2$ adrenergic receptor endocytosis, suggesting that arrestin-dependent NSF recruitment contributes to clathrin-dependent GPCR internalization (McDonald et al., 1999).

2. Cell Proliferation. $\beta$-Arrestins interface with several pathways involved in cell cycle progression, leading to context-dependent effects on cell proliferation. Primary calvarial preosteoblasts from $\beta$-arrestin2 null mice proliferate faster than wild type, and treatment with an arrestin pathway-selective biased agonist of the PTH receptor, [D-Trp $\left.{ }^{12}, \mathrm{Tyr}^{34}\right]$-bPTH(7-34), slows proliferation in a $\beta$-arrestin2-dependent manner, suggesting that arrestins restrain osteoblast proliferation (Gesty-Palmer et al., 2013). Such effects are consistent $\beta$-arrestin-dependent sequestration of inactive ERK1/2 in a microtubule-bound pool (Hanson et al., 2007a; Coffa et al., 2011b) and cytosolic retention of active ERK1/2 bound to stable GPCR-arrestin complexes (DeFea et al., 2000b; Tohgo et al., 2002; Ahn et al., 2004). Conversely, neointimal hyperplasia following carotid endothelial injury is reduced in $\beta$-arrestin 2 null mice, where its loss is associated with decreased GPCRstimulated vascular smooth muscle cell proliferation and migration, consistent with a stimulatory role for 
$\beta$-arrestin2 signaling in the proliferative response (Kim et al., 2008b). In this system, knockout of $\beta$-arrestin1 has the opposite effect, suggesting that $\beta$-arrestin 1 and 2 play opposing roles.

One mechanism by which GPCRs stimulate cell proliferation is by mediating crosstalk with the EGF receptor family of tyrosine kinase kinases, which in turn promote Ras-dependent activation of mitogenic ERK1/2 signaling. EGF receptors are activated by matrix metalloprotease-dependent shedding of EGF family growth factors, like heparin-binding EGF, which exist as preformed membrane-associated precursors (Carpenter, 2000), and GPCRs possess both G protein-dependent and $\mathrm{G}$ protein-independent mechanisms of promoting their release. EGF receptor transactivation is the major mechanism underlying ERK1/2 activation by endogenous LPA receptors in $\beta$-arrestin1/2 null murine embryo fibroblasts, clearly indicating that arrestin signaling is not essential for GPCR-EGF receptor crosstalk (Gesty-Palmer et al., 2005). In contrast, in HEK293 cells, $\beta 1$ adrenergic receptor-mediated EGF receptor transactivation and ERK1/2 activation are inhibited by downregulating $\beta$-arrestin $1 / 2$ or GRK5/6, inhibiting Src or matrix metalloprotease activity, or exposing cells to a heparinbinding EGF neutralizing antibody, suggesting that $\beta 1$ receptor-mediated EGF receptor transactivation is $\beta$-arrestin-dependent (Noma et al., 2007). Consistent with this, a GRK site mutant $\beta 1$ receptor that cannot undergo arrestin-dependent desensitization fails to transactivate EGF receptors despite exaggerated G protein signaling. In human coronary smooth muscle cells, an arrestin pathway-selective angiotensin $\mathrm{AT}_{1 \mathrm{~A}}$ receptor agonist, $\left[\mathrm{Sar}^{1}{ }^{1} \mathrm{Ile}^{4}, \mathrm{Ile}^{8}\right]$-angiotensin II (SII), induces ERK1/2 activation and proliferation by promoting EGF receptor transactivation (Miura et al., 2004), whereas in rat vascular smooth muscle both angiotensin II and SII stimulate Src-dependent EGF receptor phosphorylation on $\mathrm{Tyr}^{845}$, an effect that is lost when $\beta$-arrestin2 is downregulated by RNA interference (Kim et al., 2009). Similarly, the LH receptor activates c-Fyn in a $\beta$-arrestin2-dependent manner (Galet and Ascoli, 2008). Downregulating arrestin expression reduces the rate of $\mathrm{LH}$ receptor internalization and inhibits LH-mediated activation of c-Fyn, phosphorylation of the anti-apoptotic focal adhesion kinase, and the release of EGF-like growth factors.

$\beta$-Arrestin-bound Src has also been implicated in ERK1/2 activation by the $\beta 2$-adrenergic and neurokinin NK1 receptors (Luttrell et al., 1999; DeFea et al., 2000a). In the latter case, substance P-mediated cell survival and proliferation have been attributed to arrestin-Src signaling. Similarly, $\beta$-arrestin1-dependent activation of c-Src and EGF receptor appears to contribute to the tumor-promoting effects of prostaglandin EP2 receptors in papilloma formation (Chun et al.,
2009). Another mechanism by which $\beta$-arrestin1-Src increases cell proliferation is by activating a retinoblastoma $\mathrm{Rb}$ protein-Raf1 pathway that promotes $\mathrm{Rb}$ dissociation from E2F-responsive proliferative promoters, leading to increased E2F1 binding, transcription of S-phase genes, and cell cycle progression (Dasgupta et al., 2006). In bladder cancer, malignant transformation is associated with a thromboxane prostanoid (TP) receptor isoform switch (Moussa et al., 2008). Human bladder cancer cells express high levels of both the TP- $\beta$ receptor isoform and $\beta$-arrestin 2 . The TP- $\alpha$ and TP- $\beta$ splice variants differ only in the C terminus, with TP- $\beta$ carrying a longer tail that allows it to engage $\beta$-arrestin 2 and undergo agonist-dependent internalization (Parent et al., 1999). Expressing TP- $\beta$ in nontransformed SV-HUV urothelial cells confers agonist-dependent ERK1/2 and focal adhesion kinase phosphorylation and enhances cell proliferation, migration, and invasion in vitro, responses that are lost when $\beta$-arrestin 2 , but not $\beta$-arrestin1, is downregulated by RNA inference.

3. Nonproliferative Cell Growth. Some evidence suggests that arrestins mediate GPCR effects on cell growth. In vitro, an angiotensin $\mathrm{AT}_{1 \mathrm{~A}}$ receptor mutant with a deletion in ICL2 that inhibits G protein coupling nonetheless activates a Src-Ras-ERK1/2 pathway leading to cytosolic ERK1/2 and p90RSK activation (Seta et al., 2002). In vivo, cardiomyocyte-specific overexpression of this mutant produces more cardiomyocyte hypertrophy and fetal cardiac gene expression than comparable overexpression of the wild-type receptor (Zhai et al., 2005).

Mechanistically, $\beta$-arrestin scaffolding of the ERK1/2 cascade allows it to preferentially target cytosolic substrates involved in the control of protein translation, including the ribosomal S6 kinase, p90RSK (Aplin et al., 2007) and the MAPK-interacting kinase, MNK1, a regulator of the ribosomal protein translation initiation complex. $\beta$-Arrestin2-dependent ERK1/2 activation by the $\mathrm{AT}_{1 \mathrm{~A}}$ receptor increases phosphorylation of MNK1 and eukaryotic translation initiation factor $4 \mathrm{E}$, increasing rates of mRNA translation (DeWire et al., 2008). In addition, arrestin scaffolding of the PP2A-AKT-GSK3 $\beta$ complex is involved in regulation of mammalian target of rapamycin-dependent protein translation (Kendall et al., 2014). Increased rates of protein translation in response to angiotensin II or the arrestin pathwayselective AngII analog SII involve activation of arrestinbound pools of both ERK1/2 and AKT, AKT-mediated phosphorylation of $\mathrm{mTOR}$ and its downstream effector p70/p85 ribosomal S6 kinase, and ERK1/2 phosphorylation p90 ribosomal S6 kinase.

4. Cell Survival and Apoptosis. Several arrestin signaling complexes modulate cell survival and apoptotic pathways. In vitro, the arrestin pathway-selective PTH analog [D-Trp ${ }^{12}$, Tyr $\left.^{34}\right]$-bPTH(7-34) protects wildtype, but not $\beta$-arrestin 2 null, osteoblasts from a 
proapoptotic etoposide challenge (Gesty-Palmer et al., 2013). Likewise, the arrestin-selective $\mathrm{AT}_{1 \mathrm{~A}}$ receptor agonist, Sar $^{1} \mathrm{Ile}^{4} \mathrm{Ile}^{8}$-AngII, is anti-apoptotic in primary vascular smooth muscle (Ahn et al., 2009). Arrestin signaling also protects cardiomyocytes from undergoing apoptosis in vivo. In response to chronic isoproterenol administration, transgenic mice expressing a GRK site mutant $\beta 1$ adrenergic receptor that cannot bind arrestins develop more severe dilated cardiomyopathy, with increased left ventricular end-diastolic dimension and greater myocardial apoptosis, than wild-type $\beta 1$ receptor transgenic mice (Noma et al., 2007).

Through their scaffolding functions, arrestins are able to affect the balance between activation of proapoptotic MAPK and anti-apoptotic AKT signaling pathways. $\beta$-Arrestin-dependent assembly of the PP2A-AKT-GSK3 $\beta$ complex allows it to function as a positive regulator of PI3K/AKT-dependent survival signaling (Kendall et al., 2011). Following $\mathrm{AT}_{1 \mathrm{~A}}$ receptor activation, PP2A activity within the complex is transiently inhibited, relieving the tonic inhibition of AKT by allowing phosphorylation of $\mathrm{T}^{308}$, the activating AKT phosphorylation site, to rise. Arrestin-dependent activation of the ERK1/2 substrate p90RSK (Seta et al., 2002; Aplin et al., 2007) acts in concert with the PI3KAKT pathway to downregulate phospho-BAD, inducing anti-apoptotic cytoprotective effects in rat vascular smooth muscle (Ahn et al., 2009). Along with these prosurvival effects on AKT, arrestins tonically suppress proapoptotic signaling by the stress-activated JNK and p38 MAPKs. All of the JNK MAPKs can bind to all visual $/ \beta$-arrestins, but they exhibit highest affinity for the inactive arrestin conformation, suggesting that the dominant role of arrestins is to repress basal $\mathrm{JNK}$ signaling (Song et al., 2007, 2009a). Likewise, in at least some systems, arrestins serve primarily to attenuate $\mathrm{G}$ protein-dependent p38 MAPK activation through GPCR desensitization. $\beta$-Arrestin $1 / 2$ null murine fibroblasts exhibit enhanced activation of ERK1/2, JNK1/2, and p38 MAPK in response to the chemokine CXCR2 receptor agonist, IL-8, whereas $\beta$ arrestin expression confers protection from oxidative burst-induced cell death by attenuating JNK/p38MAPK activation (Zhao, et al., 2004). In murine embryo fibroblasts, $\beta$-arrestin expression increases resistance to serum deprivation-induced apoptosis by increasing AKT and dampening basal ERK1/2 and P38 MAPK pathway activity (Yang et al., 2012).

Although much evidence suggests that on balance, arrestin scaffolds promote cell survival, arrestins may mediate proapoptotic signals under certain conditions. By sequestering $\mathrm{Mdm} 2, \beta$-arrestin2 restricts its access to other substrates. $\mathrm{Mdm} 2$ is a major negative regulator of the p53 tumor suppressor, because ubiquitination of p53 by Mdm2 promotes its proteosomal degradation. Arrestin binding attenuates p53 ubiquitination, increasing p53 abundance, and enhancing p53 signaling.
As a result, overexpressing $\beta$-arrestin 2 in HEK293 enhances, and downregulating it attenuates, p53mediated apoptosis (Wang et al., 2003). Similarly, the angiotensin receptor blocker, losartan, has been reported to attenuate neuronal damage in an animal model of cerebral ischemia by inhibiting the assembly of a $\beta$-arrestin2-ASK1-MKK4 signaling module and repressing the activation of JNK3, c-jun, and caspase-3, and the release of cytochrome $\mathrm{C}$ (Zhang et al., 2012).

Additional evidence that arrestins can trigger apoptotic cell death comes from the study of Drosophila phototransduction. Phototransduction in the fly is different from the rhodopsin-transducin-cGMP phosphodiesterase mechanism found in mammals, relying instead on a Gq-coupled pathway to activate lightsensitive Trp channels. Desensitization of Drosophila rhodopsin involves both arrestin and the diacylglycerol kinase, RdgA. Loss of function mutation of either protein results in constitutive Trp channel activation and photoreceptor cell necrosis due to excessive $\mathrm{G}$ protein signaling (Dolph et al., 1993; Alloway et al., 2000; Kiselev et al., 2000; Raghu et al., 2000). Conversely, enhancing arrestin function appears to promote photoreceptor cell apoptosis. $\mathrm{RdgC}$ is a calcium-dependent kinase that normally dissociates the rhodopsinarrestin complex. Introducing inactivating mutations of RdgC leads to retinal degeneration due to apoptosis of photoreceptor cells (Davidson and Steller, 1998). Complementary mutations that stabilize the rhodopsinarrestin complex, such as Gq loss of function or deletion of the regulatory arrestin phosphorylation domain, enhance this form of retinal degeneration. The phenotype can be rescued either by expression of a p35 caspase inhibitor or by triple inactivation of $\mathrm{G} \alpha \mathrm{q}$, arrestin, and $\mathrm{RdgC}$, suggesting that it is the result of an arrestin-dependent apoptotic signal originating from a stable rhodopsin-arrestin complex (Kiselev et al., 2000). Consistent with this, apoptotic photoreceptor cell death caused by deletion of the eye-specific PLC gene, which likewise promotes assembly of constitutive rhodopsin-arrestin complexes, can be reversed by simultaneous deletion of arrestin or inhibition of rhodopsin endocytosis (Alloway et al., 2000).

5. Cell Migration and Chemotaxis. $\beta$-arrestin scaffolds mediate GPCR effects on actin cytoskeletal rearrangement and play important roles in cell migration, chemotaxis, and cancer metastasis. GPCR-stimulated chemotaxis is dependent upon two factors: the ability to sense a chemoattractant gradient and to establish cell polarity through cytoskeletal rearrangement at the leading edge (DeFea, 2013). Arrestin-dependent GPCR desensitization and recycling are critical to the former (Tomhave et al., 1994; Aragay et al., 1998), whereas the coordinated regulation of several processes by $\beta$-arrestin scaffolds, including cofilin dephosphorylation, filamin A recruitment, MAPK activation, and regulation of small GTPases, collectively contributes 
to the cellular shape changes needed for the latter $(\mathrm{Ge}$ et al., 2003, 2004; Barnes et al., 2005; Hunton et al., 2005).

Consistent with the critical role of $\beta$-arrestin scaffolds in the spatial control of cytoskeletal dynamics, splenocytes derived from $\beta$-arrestin 2 null mice exhibit strikingly impaired chemotatic responses to stromal cell-derived factor-1, CXC chemokine ligand 12 (Fong et al., 2002). Downregulation of either $\beta$-arrestin 1 or 2 in MDA-MB-468 cells inhibits PAR2-stimulated cofilin dephosphorylation and chemotaxis, suggesting that $\beta$-arrestin scaffolding of the cofilin-chronophin-LIM kinase complex is necessary for the localized generation of free barbed ends on actin filaments that produce filament extension and generate membrane protrusions (Zoudilova et al., 2007, 2010). Similarly, assembly of a complex containing the angiotensin $\mathrm{AT}_{1 \mathrm{~A}}$ receptor, $\beta$-arrestin, ERK1/2, and the actin filament-bundling protein, filamin $\mathrm{A}$, is involved in the formation of membrane ruffles in Hep2 cells (Scott et al., 2006).

Arrestin-dependent targeting of MAPKs also plays a role. During PAR2-induced chemotaxis, PAR2arrestin-ERK1/2 complexes localize to the leading edge of the cell, where ERK1/2 activity is required for actin cytoskeletal reorganization (Ge et al., 2003). In HeLa and HEK293 cells, overexpression/downregulation of $\beta$-arrestin 2 reciprocally enhances/attenuates activation of both p38 MAPK and ERK1/2 by the chemokine receptor CXCR4, and inhibition of p38MAPK blocks $\beta$-arrestin2-dependent chemotaxis, suggesting a role for arrestin scaffolding of p38MAPK in CXCR4stimulated cell migration (Sun et al., 2002). Arrestindependent scaffolding of p38 MAPK has likewise been implicated in control of cell polarization, actin bundle formation, and internalization of plateletactivating factor receptors in polymorphonuclear neutrophils (McLaughlin et al., 2006). Arrestin-dependent regulation of the small GTPase, RalA, through its interaction with Ral-GDS, has been implicated in formyl-Met-Leu-Phe receptor-stimulated membrane ruffling (Bhattacharya et al., 2002), Ral-dependent activation of PLC $\delta 1$ by the angiotensin $\mathrm{AT}_{1 \mathrm{~A}}$ receptor (Godin et al., 2010), and LPA receptor-mediated proliferation and migration of breast cancer cells (Li et al., 2009). Regulation of RhoA via the interaction between $\beta$-arrestin 1 and ARFGAP21 promotes angiotensin $\mathrm{AT}_{1 \mathrm{~A}}$ receptor-dependent RhoA activation and membrane ruffling (Anthony et al., 2011).

6. Modulation of the Immune Response. In immune system, $\beta$-arrestin scaffolds perform key roles through the negative regulation of $\mathrm{G}$ protein-mediated responses, promotion of chemotaxis, regulation of exocytosis and degranulation, and signal dampening through sequestration of pathway components (Jiang et al., 2013).

Within the innate immune system, $\beta$-arrestin 2 null neutrophlis show enhanced CXCR2-mediated $\mathrm{Ca}^{2+}$ signaling and superoxide generation, reflecting the loss of $\beta$-arrestin-dependent desensitization ( $\mathrm{Su}$ et al., 2005). $\beta$-arrestins regulate macrophage chemotaxis both by desensitizing chemokine CCL2-induced $\mathrm{Ca}^{2+}$ signaling and by scaffolding ERK1/2-dependent assembly of the actin cytoskeleton in pseudopodia (Aragay et al., 1998; Ge et al., 2003; Cheung et al., 2009). In polymorphonuclear leukocytes, $\beta$-arrestin1-bound c-Hck and c-Fgr regulate IL-8 CXCR1 receptorstimulated granule exocytosis (Barlic et al., 2000), similar to the reported role of a $\beta$-arrestin1-c-Yes complex in the control of endothelin-1-stimulated translocation of exocytic granules containing the glucose transporter GLUT4 (Imamura et al., 2001). Isolated polymorphonuclear leukocytes lacking $\beta$-arrestin 2 exhibit increased basal and lipopolysaccharide-stimulated release of the inflammatory cytokine TNF- $\alpha$ and IL-6 (Basher et al., 2008), perhaps due to the loss of tonic inhibition of $\mathrm{NF}_{\kappa} \mathrm{B}$ transcriptional pathways by $\beta$-arrestin2dependent sequestration of $\mathrm{I} \kappa \mathrm{B} \alpha$ and $\mathrm{I} \kappa \mathrm{B}$ kinases (Witherow et al., 2004). $\beta$-Arrestin2 also negatively regulates the activity of natural killer cells by recruiting SHP-1 and SHP-2 to the inhibitory receptor KIR2DL1 (Yu et al., 2008).

$\beta$-Arrestin scaffolds also play important regulatory roles within $\mathrm{T}$ and $\mathrm{B}$ cells of the adaptive immune system. T cell receptor activation by major histocompatibility complex antigens leads to activation of a cAMP-PKA-Csk pathway in lipid rafts that inhibits proximal T cell signaling (Bjorgo et al., 2010, 2011). Full $T$ cell activation requires the binding of costimulatory molecules to CD28, which uses $\beta$-arrestins to recruit the cAMP phosphodiesterase PDE4 into lipid rafts, relieving PKA-dependent phosphorylation of Csk and allowing $\mathrm{T}$ cell activation to proceed (Abrahamsen et al., 2004; Baillie and Houslay, 2005; Bjorgo et al., 2010). As in macrophages, $\beta$-arrestin 2 null $\mathrm{CD} 4^{+} \mathrm{T}$ cells exhibit impaired chemotactic migration (Walker et al., 2003; Raghuwanshi et al., 2008; Walker and DeFea, 2014). By regulating histone H4 acetylation at the Bcl2 locus, $\beta$-arrestin1 enhances Bcl2 expression in both naive and activated $\mathrm{CD} 4^{+} \mathrm{T}$ cells, promoting $\mathrm{T}$ cell survival and inhibiting apoptosis following cytokine withdrawal (Kang et al., 2005; Shi et al., 2007).

7. Developmental Regulation. Arrestins play important roles in embryological development, perhaps reflecting their interaction with non-GPCR elements of the Shh-Smoothened, Wnt, and Notch signaling pathways (Kovacs et al., 2009). As mentioned, $\beta$ arrestins bind Smoothened, the non-G protein-coupled seven-transmembrane receptor component of the Shh signaling pathway, and data from zebrafish suggest that $\beta$-arrestin2 functions in the Hedgehog pathway between Smoothened and its downstream transcription factor targets, $\mathrm{Su}(\mathrm{fu})$ and Gli1, to promote Shh-dependent transcription (Wilbanks et al., 2004). 
Knockdown of $\beta$-arrestin 2 blocks expression of several Shh-regulated genes and phenocopies developmental defects observed in Smoothened loss-of-function mutants, while restoring $\beta$-arrestin 2 or constitutively activating the Hedgehog pathway downstream of Smoothened rescues the defect. Interestingly, $\beta$-arrestin1 has no effect on the expression of Shhregulated genes in zebrafish, but nonetheless plays a key role in embryologic development (Yue et al., 2009). Zebrafish embryos lacking $\beta$-arrestin 1 fail to undergo hematopoiesis and exhibit severe posterior defects resulting from downregulation of $c d x 4$, a homeobox transcription factor that specifies the hematopoietic lineage by modulating hox gene expression. Hematopoiesis can be rescued either by reintroducing $\beta$ arrestin1 or injecting $c d x 4$, hoxa $9 a$, or hoxb4a mRNA. The mechanism appears to involve sequestration of the polycomb group (PcG) recruiter YY1 by nuclear $\beta$-arrestin1, which relieves PcG-mediated repression of cdx4-hox pathway.

The binding of $\beta$-arrestins to Dsh in the Wnt-Fz signaling pathway is involved in the regulation of canonical Wnt signaling in Xenopus, where knockdown of $\beta$-arrestin 2 reduces $\beta$-catenin signaling and blocks Wnt8- or Dsh-induced axis duplication (Bryja et al., 2007). $\beta$-Arrestin 2 is also required for convergent extension during Xenopus axis elongation, a process mediated by the noncanonical Wnt/planar cell polarity pathway (Kim et al., 2008a). During convergent extension, $\beta$-arrestin 2 and the Ryk receptor tyrosine kinase cooperatively mediate endocytosis of Fz7 and Dsh after Wnt11 stimulation, a process involving activation of Rac1 (Bryja et al., 2008). Without this, $\beta$-arrestin2deficient mesoderm fails to polarize and intercalate with wild-type mesoderm at the embryonic midline (Kim et al., 2008a).

During Drosophila development, the single nonvisual arrestin homolog, Kurtz, regulates the functions of Notch, a single-transmembrane-spanning receptor involved in the process of lateral inhibition (Chastagner et al., 2008). Notch ligands are transmembrane proteins that initiate juxtacrine signals resulting in proteolysis of Notch and translocation of the free Notch intracellular domain to the nucleus where it acts as a transcriptional regulator (Kopan and Ilagan, 2009). The single Notch receptor in flies is regulated via a complex with Kurtz, wherein Kurtz promotes ubiquitination of Notch by the E3 ligase, Deltex, in much the same manner that $\beta$-arrestins regulate the ubiquitination of several mammalian GPCRs (Mukherjee et al., 2005).

8. Central Nervous System Function, Learning, and Behavior. G protein-coupled metabotropic neurotransmitter receptors are critical to central nervous system function, and arrestin-dependent scaffolding and GPCR desensitization have been shown to be important in diverse central nervous system processes. The original phenotype described in $\beta$-arrestin 2 null mice was impaired $\mu$ opioid receptor (MOR) desensitization leading to prolonged analgesic effects of morphine, indicating that arrestin-dependent desensitization was the principal determinant of opiate duration of action (Bohn et al., 1999, 2000). Subsequent work indicated that some nonanalgesic effects of opiates, e.g., MOR-mediated constipation, respiratory suppression and physical dependence (Raehal et al., 2005, 2011; Raehal and Bohn, 2011), and $\kappa$ opioid receptormediated dysphoria (Bruchas et al., 2006; Redila and Chavkin, 2008), were diminished in the absence of $\beta$-arrestin 2 , suggesting that $\beta$-arrestin 2 signaling may underlie these responses.

Dopaminergic neurotransmission in the central nervous system regulates behavioral responses such as locomotor activity and neural reward mechanisms, and several lines of evidence suggest that arrestin-signaling complexes regulate dopamine-dependent behaviors. In striatum, the $\beta$-arrestin2-PP2A-AKT-GSK3 $\beta$ complex modulates D2 dopamine receptor-mediated behaviors by tonic repression of $\beta$-catenin signaling. GSK3 $\beta$ phosphorylates $\beta$-catenin, accelerating its degradation. Thus, striatal extracts from $\beta$-arrestin 2 null mice have higher levels of $\beta$-catenin, resulting from the loss of signalsome-mediated GSK3 $\beta$ activation (Beaulieu et al., 2005, 2008). Mice lacking $\beta$-arrestin2 exhibit a reduced locomotor hyperactivity response when striatal dopamine signaling is increased either by administration of the drug apomorphine or by dopamine transporter knockout. Inhibiting PP2A or GSK3 $\beta$ produces similar effects in wild-type mice. Interestingly, at therapeutic concentrations, lithium disrupts the $\beta$-arrestin2PP2A-AKT complex, leading to GSK3 $\beta$ inhibition, the mechanism by which it exerts its mood-stabilizing effects (Beaulieu et al., 2008).

$\beta$-Arrestin signaling has also been implicated in processes related to learning and memory. In mice, deletion of $\beta$-arrestin 2 , but not $\beta$-arrestin 1 , results in deficits in plasticity mediated selectively by group I metabotropic glutamate receptors (mGluRs) in CA3 and CA1 pyramidal neurons (Eng et al., 2016). mGluR1 modulation of intrinsic conductances as well as nonmGluR-mediated long-term potentiation is preserved in $\beta$-arrestin 2 null mice, indicating that the arrestin dependency is specific to a subset of mGluR-mediated responses. Experiments performed using pharmalogical inhibitors implicate the $\beta$-arrestin 2 cargos c-Src and ERK1/2 in the effects, suggesting that arrestin scaffolds are involved in regulating mGluR changes in synaptic strength.

\section{Silent Scaffolds and Tonic Effects on Pathway Activity}

A fourth property of visual $/ \beta$-arrestins that contributes to their effectiveness as scaffolds is simply that they are relatively abundant in relation to the GPCRs they desensitize and catalytically active signaling 
proteins they carry as cargos. The ability to shuttle between abundant low-affinity binding sites on microtubules and smaller numbers of high-affinity binding sites on activated GPCR permits them to sequester key pathway intermediates away from their site of activation and dampen basal pathway activity until called to respond to an extracellular stimulus.

The dichotomous effects of $\beta$-arrestins on ERK1/2 and JNK pathway activation underscore the point that dampening basal signaling pathway may be as important to arrestin function as their ability to support pathway activation. As discussed, ERK1/2 has significant affinity for only the microtubule-bound and receptor-bound arrestin conformations, whereas cRaf1 and activated ERK1/2 have highest affinity for the receptor-bound arrestin conformation (Luttrell et al., 2001; Coffa et al., 2011b). The result is that inactive ERK1/2 can be sequestered by a microtubule-bound arrestin pool until called to a receptor, where cRaf-1 activation by plasma membrane-delimited effectors, e.g., Ras, initiates the ERK1/2 activation cascade (Jafri et al., 2006). In contrast, activation of the ASK1-MKK4/7-JNK3 cascade does not appear to be a GPCR-regulated process. Although the original study reported that stimulation of angiotensin $\mathrm{AT}_{1 \mathrm{~A}}$ receptors activated JNK3 and caused it to colocalize with $\beta$-arrestin2 in endosomal vesicles (McDonald et al., 2000 ), later work performed using the $\beta 2$ adrenergic receptor found no evidence of receptor-mediated JNK3 activation under conditions where ERK1/2 was being robustly activated via the $\beta$-arrestin2 pathway (Breitman et al., 2012). In fact, JNK3 affinity is highest for inactive arrestin mutants that do not bind GPCRs, further supporting the concept that whereas $\beta$-arrestin-dependent ERK1/2 activation is receptordependent, JNK3 activation is not (Song et al., 2006; Breitman et al., 2012). Instead, the principal effect of arrestins on JNK appears to be to dampen pathway activity. Although all four visual $/ \beta$-arrestins bind JNK3 comparably and redirect it from the nucleus, where it spontaneously localizes, to the cytoplasm (Song et al., 2007 ), only $\beta$-arrestin 2 scaffolds JNK3 activation. In the other arrestin isoforms, the properties of JNK3 binding and activation are dissociated (Song et al., 2009a; Zhan et al., 2011b). Even though JNK3 associated with cytosolic $\beta$-arrestin 2 is active, the $\beta$-arrestin 2 NES keeps JNK3 out of the nucleus. Thus, whereas arrestins target ERK1/2 to specific substrates through GPCR-dependent activation and tethering to GPCRarrestin complexes, arrestin binding appears to keep JNK3 away from both GPCRs and its nuclear transcription factor targets (Lin and Defea, 2013).

Similar signal-dampening effects of arrestin binding have been reported for other arrestin effectors. Downregulating $\beta$-arrestin 1 in HeLa cells increases NF- $\kappa \mathrm{B}$ activation by TNF- $\alpha$ consistent with the hypothesis that arrestins tonically inhibit NF- $\kappa \mathrm{B}$ signaling by protecting $\mathrm{I} \kappa \mathrm{B} \alpha$ from degradation (Witherow et al., 2004). In HEK-293 cells, downregulating arrestin expression attenuates TLR4-mediated ERK1/2 activation while at the same time enhancing $\mathrm{NF}_{\kappa} \mathrm{B}$ reporter activity, indicating that arrestins exert opposing effects on the ERK1/2 and $\mathrm{NF}_{\kappa} \mathrm{B}$ pathways (Fan et al., 2007). Another example is the binding of nuclear $\beta$-arrestin 1 to PPAR $\gamma$, which competes for RXR $\alpha$ binding and attenuates PPAR $\gamma$-RXR $\alpha$-dependent transcription (Zhuang et al., 2011).

\section{E. Self-Association of Arrestins}

Although GPCR-bound arrestins appear to be monomeric, three of the four visual $/ \beta$-arrestins are capable of self-association in solution (Chen et al., 2014), with only cone arrestin being a constitutive monomer. Despite this shared property, the structure, regulation, and physiologic significance of arrestin multimers differ between visual arrestin and the $\beta$-arrestins.

Visual arrestin forms tetramers both in crystal form and in solution; however, the crystallized and solution tetramers differ in organization (Granzin et al., 1998; Hirsch et al., 1999; Hanson et al., 2007b; Kim et al., 2011). In solution structure, visual arrestin tetramers adopt a circular conformation in which all selfassociation interfaces are engaged, explaining why self-association stops at tetramers. As importantly, in the solution tetramer all visual arrestin elements involved in rhodopsin binding are either engaged or shielded by other protomers, which is why only monomeric visual arrestin can bind rhodopsin (Hanson et al., 2007b; Beyriere et al., 2015; Kang et al., 2015). After rhodopsin, visual arrestin is the most abundant protein in the rod outer segment, and a delicate balance between spontaneous visual arrestin self-association and light-dependent translocation works together to keep a constant supply of active rhodopsin-binding visual arrestin monomer in the outer segment (Gurevich et al., 2011). In this system, the tetrameric visual arrestin complex appears to function as a storage form, whose role is to protect photoreceptor cells from potentially toxic effects of excess free monomeric visual arrestin (Schubert et al., 1999; Hanson et al., 2007b; Kim et al., 2011). To maintain light sensitivity at the single photon level, rods must contain high enough levels of visual arrestin to quench signaling by all expressed rhodopsin. Loss of visual arrestin or defective rhodopsin phosphorylation resulting from deletion of the rhodopsin kinase, GRK1, led to photoreceptor death, presumably due to excessive signaling (Song et al., 2013). Paradoxically, introducing an enhanced phosphorylation-independent visual arrestin mutant that is impaired in self-association into a GRK1/visual arrestin null background, which would be expected to rescue the defect, produces biphasic effects. When expressed at $50 \%$ of wild-type levels, the expected rescue is observed, but at higher levels of expression 
the mutant itself is toxic. The difference appears to relate to its inability to form inactive cytosolic multimers. At physiologic levels of expression, monomeric wild-type visual arrestin comprises only about $1.5 \%$ of the total arrestin complement of dark-adapted rods, with the balance sequestered in dimeric or tetrameric form. Expressing the mutant at $240 \%$ of wild-type levels increases free visual arrestin about threefold over physiologic levels, causing accelerated photoreceptor degeneration (Huang et al., 2010; Moaven et al., 2013; Song et al., 2013). Thus, in the retina, visual arrestin self-association appears to provide a mechanism for maintaining arrestin levels high enough to meet physiologic needs while keeping monomeric arrestin levels low.

The $\beta$-arrestins likewise readily form homo- and heterooligomers at physiologic levels of expression (Storez et al., 2005; Milano et al., 2006). As noted, the abundant cellular metabolite, IP6, which inhibits self-association of visual arrestin, greatly enhances $\beta$-arrestin 1 self-association (Hanson et al., 2008). In the presence of IP6, $\beta$-arrestin2 forms dimers wherein IP6 connects the $\mathrm{C}$ domains of two adjacent molecules, whereas $\beta$-arrestin 1 forms infinite chains with IP6 connecting the concave sides of the $\mathrm{N}$ and $\mathrm{C}$ domains of adjacent protomers (Chen et al., 2014). As both IP 6 binding sites on $\beta$-arrestin $1 / 2$ overlap the receptor binding sites, it is likely that oligomeric $\beta$-arrestins represent a storage form, as in the case of visual arrestin (Milano et al., 2006). Because $\beta$-arrestin 1 mutants with impaired IP6 binding show increased nuclear localization, oligomerization may also serve to keep constitutively bound arrestin cargos, like JNK3, out of the nucleus. The formation of $\beta$-arrestin $1 / 2$ hetero-oligomers may have a similar function, in that the NES of the $\beta$-arrestin2 protomer may help sequester $\beta$-arrestin1 in the cytosol (Storez et al., 2005). Interestingly, a $\beta$-arrestin2 mutant that does not bind IP6 was also found to lack affinity for $\mathrm{Mdm} 2$ and could not suppress Mdm2-dependent degradation of $\mathrm{p} 53$, suggesting that $\mathrm{Mdm} 2$ sequestration is a specific function of dimeric $\beta$-arrestins (Boularan et al., 2007). Another report found that $K^{285} \mathrm{~A} / \mathrm{R}^{286} \mathrm{~A}$ mutations in the polar core of $\beta$-arrestin 1 that reduced formation of homodimers also impaired $\beta 2$-adrenergic receptor binding, ERK1/2 binding, and $\beta 2$-adrenergic receptordependent ERK1/2 activation (Xu et al., 2008). Whether this indicates $\beta$-arrestin 1 self-association is involved in controlling arrestin-dependent scaffolding of the ERK1/2 complex, or that common residues are involved in both $\beta$-arrestin oligomerization and scaffolding, remains to be determined.

\section{Visual $/ \boldsymbol{\beta}$-Arrestins as Therapeutic Targets}

\section{A. Orthosteric and Allosteric Modulation of GPCR Signaling}

Because they are involved in the coordination and control of nearly every physiologic process, it is not surprising that GPCRs are the most commonly exploited drug targets by a wide margin (Lappano and Maggiolini, 2011). The visual $/ \beta$-arrestins, in turn, by virtue of their unique combination of desensitizing/ scaffolding functions, are key determinants of the balance between $\mathrm{G}$ protein and non-G protein signals stemming from GPCR activation. As the cellular responses mediated by these two distinct signaling systems differ, manipulating them independently, i.e., changing the balance between $\mathrm{G}$ protein and arrestindependent processes, might offer distinct advantages over conventional agonist or antagonist approaches (Luttrell, 2013).

GPCRs, which were originally envisioned as simple detectors of hormones in the extracellular environment (Ahlquist, 1948), then as binary switches whose proportional distribution in the off and on states is determined by the intrinsic efficacy and concentration of agonist ligands (Samama, et al., 1993), have come to be viewed more broadly as components of an allosterically regulated signal transduction machinery whose function is to bind molecules, i.e., extracellular ligands, at one location, and change shape to affect the binding and conformation of other molecules, i.e., intracellular effectors, at another location (Kenakin, 2012). Embedded within this general allosteric model of GPCR function are the concepts of pluridimensional efficacy, i.e., that GPCRs signal by engaging multiple $G$ protein and non-G protein effectors (Galandrin et al., 2007), and functional selectivity, i.e., that ligand-specific signaling patterns can emerge because of differences in the efficiency with which ligands stabilize the structural conformations that couple the receptor to each of its possible downstream effectors (Kenakin and Christopoulos, 2013).

Numerous examples of ligand bias have been described, both favoring G protein coupling over GRK phosphorylation and arrestin recruitment, and favoring arrestin coupling over $\mathrm{G}$ protein signaling, and the potential of biased ligands as therapeutics has been the subject of multiple reviews (Luttrell and Kenakin, 2011; Whalen et al., 2011; Kenakin and Christopoulos, 2013; Kenakin, 2015). It is less clear at present whether more nuanced form of bias is attainable, e.g., dissociating arrestin-dependent desensitization from arrestindependent signaling. Because some GPCRs appear able to engage arrestins in a manner that permits endocytosis without precluding heterotrimeric $\mathrm{G}$ protein coupling (Thomsen et al., 2016), it is possible that ligand structure might be able to drive both receptor internalization and prolonged $\mathrm{G}$ protein signaling by preferentially stabilizing different arrestin-docking poses.

The main attraction of ligand bias as a means to modulate arrestin function is tissue selectivity, a critical consideration for a target as ubiquitous and multifunctional as the $\beta$-arrestins. Because the ligand-GPCR complex, not just the GPCR, determines how the cell 
will respond, biased ligands can in effect create new receptors whose signaling properties differ from that of the same receptor bound to its native ligand. Although the structure of the ligand-GPCR complex will determine which effectors are engaged, the tissue distribution/expression of the receptor will determine where that activation occurs (Luttrell, 2014). Another intriguing property of such ligands is that they can activate the same effector in different ways. Biophysical measurements of the conformational signature of $\beta$-arrestin2, induced by different angiotensin $\mathrm{AT}_{1 \mathrm{~A}}$ receptor agonist peptides, show that structurally distinct ligands can produce different arrestin conformations, leading to differences in arrestin-receptor avidity and the efficiency of arrestin-dependent ERK1/2 activation (Zimmerman et al., 2012; Lee et al., 2016). Similar findings have been reported for $\mathrm{G}$ proteins, where human and salmon calcitonin activating the calcitonin receptor produce distinct $\mathrm{G} \alpha \mathrm{s}$ conformations that differ in their rate of GTP exchange and efficiency with which they activate adenylyl cyclase (Furness et al., 2016).

Modulation of arrestin function through allosteric sites on GPCRs is conceptually similar. Allosteric modulators are molecules that bind GPCR domains that are topographically distinct from the orthosteric site, leading to an increase or decrease in the ability of the orthosteric ligand to interact with the receptor and/ or modulate its ability to stabilize active receptor conformations (Kenakin and Miller, 2010). Allosteric modulators have several theoretical advantages over orthosteric ligands, including improved subtype selectivity for closely related GPCRs with conserved ligandbinding pockets (Lazareno et al., 1998; Ellis and Seidenberg, 2000) and low risk of overdose, as their effects are saturable. Because the activity of an allosteric modulator is dependent upon the presence of the endogenous ligand, its effects are tied to endogenous patterns of ligand release and less likely to produce tachyphylaxis (Kenakin, 2009; Wang et al., 2009). Additionally, allosteric modulators may possess intrinsic agonism, i.e., allo-agonism, and can bias the stimulus leading to signaling pathway-selective effects, e.g., favoring $G$ protein or arrestin coupling (Sachpatzidis et al., 2003; Zhang et al., 2005).

\section{B. Inhibiting or Ablating Arrestins}

In some settings, it may be desirable to inhibit arrestin function, either to enhance $G$ protein signaling by reducing GPCR desensitization, or to block potentially deleterious arrestin-mediated signaling. One strategy that has been exploited is to target GRKs. Interestingly, of the four widely expressed extraretinal GRKs, GRK2/3 and GRK5/6 have been shown to perform different functions with respect to GPCR desensitization and signaling (Kim et al., 2005; Ren et al., 2005; Noma et al., 2007; Zimmerman et al., 2012). Whereas downregulating GRK2/3 expression inhibits $\beta$-arrestin-dependent desensitization and internalization of the angiotensin $\mathrm{AT}_{1 \mathrm{~A}}$ and vasopressin $\mathrm{V} 2$ receptors, downregulation of GRK5/6 disproportionately reduces $\beta$-arrestin-dependent ERK1/2 activation, suggesting that isoform-selective GRK inhibitors might offer a means to modulate arrestin function. In congestive heart failure, GRK2 is upregulated as a consequence of chronic activation of the sympathetic nervous system, leading to chronic desensitization and downregulation of cardiac $\beta 1$ adrenergic receptors and a marked reduction of myocardial inotropic reserve (Cannavo et al., 2013; Woodall et al., 2014; Sato et al., 2015). In several animal models of heart failure, genetic deletion of GRK2, or its inhibition through transgenic or adenoviral expression, a $\mathrm{G} \beta \gamma$ subunit sequestrant peptide has been shown to improve functional and morphologic cardiac parameters, including enhanced responsiveness to adrenergic stimuli due to the alleviation of arrestin-dependent tachyphylaxis (Rockman et al., 1998; Harding et al., 2001; Raake et al., 2013). In addition, small-molecule inhibitors of $\mathrm{G} \beta \gamma$ subunits (Casey et al., 2010; Piao et al., 2012) or GRK2 itself (Thal et al., 2012) have shown beneficial effects in rodent heart failure models, and newer GRK isoformspecific inhibitors are in development (Homan et al., 2015).

Direct inhibitors of visual $/ \beta$-arrestins have not been described to date, although modulation of arrestin function through binding small molecules has been demonstrated in the case of IP6, which interacts with discrete binding sites in the $\mathrm{N}$ and $\mathrm{C}$ domains (Milano et al., 2006; Hanson et al., 2008). By promoting selfassociation, IP6 binding sequesters $\beta$-arrestins in an inactive cytosolic pool, away from the plasma membrane and nucleus, and changing its affinity for some cargos (Boularan et al., 2007; Xu et al., 2008). Although its highly charged nature and relatively low affinity make IP6 unsuitable as an arrestin inhibitor, its effects illustrate the feasibility of small molecules targeting arrestins.

Rather than small molecules, experimental approaches to inhibiting arrestins have involved genetic ablation or downregulation by RNA interference. $\beta$-Arrestin downregulation predictably results in decreased GPCR internalization, prolonged G proteinmediated signaling, and loss of arrestin-dependent responses, including arrestin-dependent chemotaxis (Ahn et al., 2003; Hunton et al., 2005). In vivo, $\beta$-arrestin 2 downregulation by intrathecal administration of small-interfering RNA in rats has been shown to potentiate the antinociceptive effects of opiates and attenuate opiate tolerance (Yang et al., 2011), consistent with observations made in $\beta$-arrestin2 knockout mice (Bohn et al., 1999, 2000). Arrestin-specific aptamers, oligonucleotides whose secondary and tertiary structures enable selective binding to the surface of protein targets and blockade of protein-protein 
interactions, have also been developed (Kotula et al., 2014). Administration of a $\beta$-arrestin $2-$ specific aptamer to leukemic cells has been shown to impair $\beta$-arrestindependent signaling and inhibit tumor progression in CML models and samples obtained from human patients. Conjugating the arrestin-specific aptamer to another aptamer that is tumor cell specific enhanced its effectiveness.

\section{Customizing Arrestin Function}

Many diseases result from either gain- or loss-offunction mutations that render signaling pathways insensitive to normal regulation, such as constitutive activation of GPCRs (Spiegel, 1996; Schöneberg et al., 2004) or inactivating mutations of GRK1 and visual arrestin (Baylor and Burns, 1998). One strategy for gene therapy rescue of such defects is to introduce compensatory mutations into other pathway components that restore pathway regulation. The structure of visual $/ \beta$-arrestins is such that they can be customized in a manner that enhances or removes certain functions without compromising their overall function (Gurevich and Gurevich, 2013). GPCR binding involves the concave surfaces of the $\mathrm{N}$ and $\mathrm{C}$ domains, where a relatively few residues determine receptor selectivity (Vishnivetskiy et al., 2011; Gimenez et al., 2014a), whereas most other cargo proteins interact with the outer surfaces that remain exposed on the receptorbound arrestins. Similarly, the clathrin and AP2 binding sites in the C-terminal tail do not overlap with the sites for other binding partners. Hence, mutation of these sites can readily dissociate $\beta$-arrestin-dependent desensitization, which only requires recruitment to GPCRs on the plasma membrane, from clathrindependent GPCR endocytosis, which requires clathrin and AP2 binding (Goodman et al., 1997; Laporte et al., 2000).

Several customized arrestins have been developed, and one has even been tested in gene therapy rescue experiments (Gurevich et al., 2014). As noted, arrestin activation is triggered by the interaction between phosphate residues on the GPCR $\mathrm{C}$ terminus and the arrestin phosphate sensor, and point mutations within the phosphate sensor produce an arrestin molecule that binds activated receptors independent of their phosphorylation state (Gurevich and Benovic, 1995, 1997). One such enhanced arrestin 1 mutant has been shown to partially compensate for the loss of GRK1, facilitating the rate of rod recovery and improving rod survival more efficiently that equivalently overexpressed wildtype arrestin-1 (Song et al., 2009b). Similar enhanced phosphorylation-independent versions of all visual/ $\beta$-arrestins have been constructed and shown to effectively desensitize several GPCRs without receptor phosphorylation (Kovoor et al., 1999; Celver et al., 2001, 2002), although their utility as therapeutic agents outside the retina would probably also require mutagenic manipulation of their GPCR selectivity to avoid global attenuation of $\mathrm{G}$ protein signaling.

All visual $/ \beta$-arrestins except cone arrestin are capable of self-association, and arrestin oligomers most likely represent a cytosolic storage form of the protein, as only monomeric arrestins can bind GPCRs. The capacity to self associate appears most physiologically relevant for visual arrestin, as the equilibrium between tetrameric, monomeric, and rhodopsin-bound pools protects photoreceptor cells from the toxic effects of free visual arrestin monomers (Song et al., 2013). Targeted elimination of two homologous phenylalanine residues in bovine and mouse visual arrestin generates selfassociation deficient mutants, indicating that the equilibrium between the storage and reactive forms of visual arrestins is a factor that can be manipulated independent of other arrestin functions (Hanson et al., 2008; Kim et al., 2011). Unlike visual arrestin, selfassociation of $\beta$-arrestins is enhanced by IP 6 binding, and the behavior of $\beta$-arrestin mutants with impaired IP6 binding suggests that self-association plays a regulatory role (Hanson et al., 2008; Chen et al., 2014 ). By virtue of its NLS, monomeric $\beta$-arrestin 1 will readily enter the nucleus. In contrast, $\beta$-arrestin 1 oligomers are predominantly cytosolic, allowing them to sequester nuclear cargos like JNK3 (Milano et al., 2006). Although $\beta$-arrestin 2 is normally cytosolic due to the presence of a NES, IP6-dependent oligomerization appears to regulate its interaction with $\mathrm{Mdm} 2$, such that elimination of IP6-binding residues interferes with its tonic Mdm2-dependent inhibition of p53 signaling and antiproliferative effects (Boularan et al., 2007).

Arrestin's scaffolding functions can also be manipulated. Although GPCR binding is essential for $\beta$-arrestin-dependent activation of ERK1/2 (Coffa et al., 2011b), $\beta$-arrestin 2 -mediated facilitation of JNK3 phosphorylation is receptor-independent, suggesting that free cytosolic arrestin performs this function (Song et al., 2006; Zhan et al., 2011b). Although the binding sites of MAPKs remain poorly defined, several mutated forms of $\beta$-arrestin that affect MAPK signaling have been created. An $\mathrm{R}^{307} \mathrm{~A}$ mutant of $\beta$-arrestin 1 has impaired cRaf-1 binding but binds normally to MEK1/2 and ERK1/2 (Coffa et al., 2011a). When expressed in $\beta$-arrestin1/2 null fibroblasts, this mutant retains its ability to engage phosphorylated $\beta 2$-adrenergic receptors, but fails to reconstitute arrestin-dependent ERK1/ 2 activation. Thus, it might function as a dominantnegative inhibitor of arrestin-dependent ERK1/2 activation while not impairing receptor desensitization. In the case of JNK3, both wild-type $\beta$-arrestin 2 and a seven-residue deletion mutant in the hinge region that preferentially binds microtubules (Vishnivetskiy et al., 2002; Hanson et al., 2007a) efficiently scaffold the ASK1-MKK4-JNK3 module, whereas the preactivated $\beta$-arrestin2 mutant that exhibits enhanced GPCR binding (Celver et al., 2002) and a receptor-binding deficient 
mutant wherein receptor-binding residues were mutated to alanine (Vishnivetskiy et al., 2011; Gimenez et al., 2012b) do not support JNK3 activation (Breitman et al., 2012). The latter mutant is able to bind ASK1, MKK4, and JNK3 efficiently, but, like $\beta$-arrestin1, does not promote JNK3 activation and does not recognize GPCRs. Thus, it behaves as a silent scaffold, inhibiting JNK3 activation by sequestering ASK1, MKK4, and JNK3 away from productive scaffolds while not transitioning to the low JNK3 affinity conformation induced by receptor binding, and might serve to suppress proapoptotic signaling in cells. Conversely, a 25-amino-acid miniscaffold derived from $\beta$-arrestin2 is sufficient to bind ASK1, MKK4/7, and JNK3 and scaffold JNK3 activation in cells, suggesting that arrestin-derived peptides can be used to manipulate antiproliferative and proapoptotic JNK signaling in cells (Zhan et al., 2016).

\section{Potential Therapeutic Applications}

Regardless of the approach used to manipulate visual $/ \beta$-arrestins, their universality and myriad functions must raise concerns when thinking of them as drug targets. Even functionally selective orthosteric ligands, which derive specificity from the cell/tissue distribution of the cognate GPCR, might produce unexpected on-target effects as a result of unbalanced receptor activation (Appleton and Luttrell, 2013). Still, data from animal models offer several examples in which selective attenuation or enhancement of arrestin function might be beneficial.

1. Potential Benefits of Reducing Arrestin Activity. Inhibiting arrestin function would have two expected consequences: $\mathrm{G}$ protein-dependent signaling would be enhanced by the impairment of homologous desensitization, and signals generated by arrestin scaffolds would be reduced. Depending on the setting, both effects can be therapeutically desirable. As noted, $\beta$-arrestin 2 null mice experience prolonged analgesic effects of morphine due to the loss of MOR desensitization (Bohn et al., 1999, 2000), while at the same time encountering less constipation, respiratory suppression, and physical dependence (Raehal et al., 2005, 2011; Raehal and Bohn, 2011). To capitalize on this clinically in the form of more efficacious and safer narcotic analgesics, it would be desirable to decrease the arrestin-dependent efficacy of opioid receptor ligands (DeWire et al., 2013; Schmid et al., 2013; Zhou et al., 2013). In murine models, a weakly G proteinbiased MOR agonist, TRV130, reportedly achieves favorable separation between analgesic efficacy and gastrointestinal and respiratory side effects (DeWire et al., 2013). This compound has entered human clinical trials, where it has proven to be a very potent analgesic, but has yet to show the expected reduction in side effects (Soergel et al., 2014), raising the question of whether biased agonists at MOR can dissociate analgesia from respiratory depression/constipation, or if a greater degree of bias is necessary to translate into human therapeutic responses (Luttrell et al., 2015). Analogous $\mathrm{G}$ protein-biased $\kappa$ opioid receptor agonists are under development (Schmid et al., 2013; Zhou et al., 2013; White et al., 2014, 2015), aimed at dissociating beneficial Gi/o-mediated antinociceptive effects (Gullapalli and Ramarao, 2002), from the putatively arrestin-mediated undesirable effects of dysphoria, sedation, diuresis, hallucination, and depression (Bruchas et al., 2006; Land et al., 2008; Redila and Chavkin, 2008).

Excessive dopaminergic neurotransmission underlies several cognitive disorders, including schizophrenia and attention deficit hyperactivity disorder. As a result, D2 dopamine receptor antagonists are effective neuroleptics. D2 receptors modulate Gs-cAMP signaling through coupling to $\mathrm{Gi} / \mathrm{o}$ family $\mathrm{G}$ proteins, and engage AKT-GSK3 signaling via arrestin scaffolding of a $\beta$-arrestin2-PP2A-AKT-GSK3 $\beta$ complex (Beaulieu et al., 2005, 2008). Locomotor hyperactivity induced by the dopaminergic drug apomorphine, a D2 receptor agonist, is reduced in $\beta$-arrestin 2 null mice, implicating arrestin-dependent signaling in D2 receptor-dependent behaviors, and the mood stabilizer lithium acts by destabilizing the $\beta$-arrestin2-PP2A-AKT-GSK3 $\beta$ complex, which enhances AKT-dependent inhibition of GSK3 $\beta$ (Beaulieu et al., 2008). Strikingly, the clinical efficacy of nearly all mood-stabilizing drugs correlates better with their ability to inhibit $\beta$-arrestin2-dependent signaling than with their effects on D2 receptor-G protein signaling (Masri et al., 2008; Gao et al., 2015). Although different classes of antipsychotics vary in D2 receptor-G protein agonist/antagonist efficacy, they share the ability to block $\beta$-arrestin 2 recruitment to D2 receptors.

$\beta$-Arrestin1-dependent scaffolding of cPLA2 underlies the cutaneous flushing side effect of nicotinic acid that limits its utility in the treatment of hyperlipidemia. Activation of GPR109A by niacin lowers serum-free fatty acids by activating $G_{i} / G_{o}$ signaling. At the same time, it stimulates cPLA2-dependent production and release of the vasodilator, prostaglandin $\mathrm{D}_{2}$, producing an increase in cutaneous blood flow and flushing (Kather et al., 1983; Pike, 2005). $\beta$-arrestin1-cPLA2 scaffolding underlies the GPR109A-dependent release of arachidonate, the precursor of prostaglandin $D_{2}$ (Walters et al., 2009). Although deletion of $\beta$-arrestin1 or 2 has no effect on nicotinic acid-induced changes in free fatty acid levels, deletion of $\beta$-arrestin 1 blocks niacin-stimulated flushing in a murine model, suggesting that a G protein-selective GPR109A agonist would mimic the effects of niacin on lipid metabolism while eliminating flushing. Consistent with this, the GPR109A agonist, MK-0354, which decreases serumfree fatty acids without causing flushing (Semple et al., 2008), does not promote arrestin recruitment in vitro. 
One of the mainstays of asthma therapy is the use of inhaled bronchodilators acting as agonists of $\beta 2$ adrenergic receptors on bronchial smooth muscle, both as short-acting rescue inhalers and as long-acting preparations. Although long-acting $\beta 2$ agonists, when used in combination with anti-inflammatory inhaled corticosteroids, have been shown to improve asthma control overall, the chronic desensitization of $\beta 2$ receptors resulting from their use may reduce the effectiveness of rescue inhalers and increase mortality from acute asthma attacks (Oppenheimer and Nelson, 2008). One of the curious phenotypes of $\beta$-arrestin 2 null mice is that they do not accumulate $\mathrm{T}$ helper 2 cells in the airway or develop airway inflammation in an ovalbumin sensitization and inhaled challenge model of allergic asthma (Walker et al., 2003). Given the proposed role of $\beta$-arrestin scaffolds in $\mathrm{T}$ cell migration (Fong et al., 2002; Sun et al., 2002), it appears likely that $\beta$-arrestins exacerbate the asthma phenotype both by desensitizing bronchodilator receptors leading to enhanced bronchospasm and promoting chemokine receptor-mediated airway inflammation. Consistent with this, the bronchodilatory effects of PAR2, which are mediated via prostaglandin E2 production, are preserved in $\beta$-arrestin 2 null mice, whereas lung eosinophil and $\mathrm{CD}^{+} \mathrm{T}$ cell infiltration, and production of IL-4, IL-6, IL-13, and TNF- $\alpha$, are lost (Nichols et al., 2012). Hence, inhibiting arrestin function in the airway might be expected to augment treatment of allergic asthma (Walker and DeFea, 2014). If $\beta$-arrestins perform similar roles with respect to PAR1 and PAR2 regulation of ion transport, mucosal permeability, epithelial cell motility, immune cell recruitment, and nociception in the gut, then inhibiting arrestin function in the gut may have value in the treatment of irritable bowel syndrome and colitis (Ramachandran et al., 2012).

Because of their roles in supporting cell growth, survival, and migration, $\beta$-arrestin scaffolds also appear to contribute to a more aggressive cancer phenotype. The finding that $\beta$-arrestin 2 knockdown reverses TP- $\beta$ receptor-induced effects on bladder cancer cell proliferation, migration, and invasion of SV-HUV urothelial cells in vitro (Parent et al., 1999) is consistent with data from a murine xenograft model of TP- $\beta-$ dependent human bladder cancer, where a TP- $\beta$ receptor antagonist delays tumor growth and prolongs survival (Moussa et al., 2008). Upregulation of LPA1 and LPA2 receptors, $\beta$-arrestins 1 and 2 , Ral, and RalGDS occurs in more advanced stages of human breast cancer, and knockdown of either $\beta$-arrestin or Ral expression inhibits LPA-stimulated migration and invasion of MDA-MB-231 breast cancer cells in vitro ( $\mathrm{Li}$ et al., 2009). Upregulation of endothelin endothelin type A receptors and $\beta$-arrestin 1 is associated with advanced grades of human ovarian cancer, whereas silencing $\beta$-arrestin expression in vitro inhibits endothelin type
A receptor activation of c-Src, ERK1/2, Akt, and EGF receptor and blocks $\beta$-catenin-dependent activation of a T cell-specific factor/LEF reporter (Rosano et al., 2009). In a tumor xenograft model, ovarian tumor cells expressing an inhibitory $\mathrm{S}^{412} \mathrm{D}$ mutant of $\beta$-arrestin1 mutant develop fewer metastases, suggesting that arrestin signaling contributes to ovarian tumorigenesis and progression. Papilloma formation following exposure to tumor-promoting phorbol esters results from prostaglandin E2 production, which triggers EGF receptor transactivation by a prostaglandin $\mathrm{E} 2$ receptor- $\beta$ arrestin1-c-Src complex (Chun et al., 2009). Thus, arrestin-dependent signaling may be a therapeutic target in a host of milignancies.

2. Benefits of Promoting Arrestin Activity. Not all in vivo consequences of visual $/ \beta$-arrestin signaling are deleterious, however. In several settings, restoration of arrestin-dependent desensitization or selective activation of arrestin-dependent signaling pathways may have uniquely beneficial effects. Visual phototransduction involves a delicate balance between light sensitivity engendered by very high rhodopsin density in the rod outer segment, and rapid signal quenching achieved through GRK1 phosphorylation and visual arrestinmediated desensitization. Perturbations that result in either excessive signaling or constitutive desensitization are toxic to photoreceptor cells. Oguchi disease, a hereditary form of stationary night blindness, results from inactivating mutations in GRK1 or visual arrestin that impair photoreceptor desensitization and increase signaling (Baylor and Burns, 1998). Conversely, a ${ }^{296} \mathrm{E}$ point mutation in the opsin binding site of rhodopsin that leads to constitutive GRK1 phosphorylation and desensitization in vivo underlies one autosomal dominant form of retinitis pigmentosa ( $\mathrm{Li}$ et al., 1995). The capacity to customize arrestin function suggests that a strategy of compensatory gene therapy could be a viable means of restoring lost arrestin function (Gurevich et al., 2014). As proof-of-concept, introducing a phosphorylation-independent visual arrestin mutant into GRK1 null rods, which would replace the normal two-step process of GRK phosphorylation followed by visual arrestin binding with a one-step process that bypasses the defect, was able to suppress rhodopsin signaling and enhance photoreceptor survival, functional performance, and photoresponse recovery (Song et al., 2009b).

The roles of $\beta$-arrestins in the cardiovascular system are complex, as they appear not only to modulate vasoconstrictor tone through GPCR desensitization, but to mediate effects on vascular smooth muscle proliferation, survival, and migration (Ahn et al., 2009; Kim et al., 2009). In isolated cardiomyocytes, treatment with the arrestin pathway-selective angiotensin $\mathrm{AT}_{1 \mathrm{~A}}$ receptor agonist, $\mathrm{Sar}^{1} \mathrm{Ile}^{4} \mathrm{Ile}^{8}$-AngII, stimulates cardiomyocyte proliferation, while generating positive inotropic and lusitropic effects (Rajagopal et al., 2006; Aplin 
et al., 2007), whereas in the heart, arrestin-dependent signaling may contribute to cardiomyocyte hypertrophy while promoting cell survival (Zhai et al., 2005). In contrast, $\beta$-arrestin1-dependent ERK1/2 signaling in the adrenal gland promotes salt retention by regulating expression of the steroidogenic acute regulatory protein, StAR, the rate-limiting enzyme in aldosterone biosynthesis (Lymperopoulos et al., 2009), leading to volume retention and worsening of congestive heart failure (Bathgate-Siryk et al., 2014; Dabul et al., 2015). Because afterload reduction using conventional antagonists of angiotensin $\mathrm{AT}_{1 \mathrm{~A}}$ receptor signaling is known to promote beneficial cardiac remodeling and improve survival in congestive heart failure (Michel et al., 2016), the question arises whether, on balance, enhancing or inhibiting $\beta$-arrestin signaling would provide the most benefit. Some data suggest that the positive inotropic and lusitropic effects of $\mathrm{Sar}^{1} \mathrm{Ile}^{4} \mathrm{Ile}^{8}$-AngII in vitro translate into improved cardiac contractility in vivo. An analogous arrestin pathway-selective $\mathrm{AT}_{1 \mathrm{~A}}$ receptor agonist, TRV120027 [Sar-Arg-Val-Tyr-Ile-His-Pro-(D)Ala-OH], which also stimulates arrestin-dependent activation of Src, ERK1/2, and endothelial nitric oxide synthase in vitro, similarly improves cardiomyocyte contractility in preclinical animal models (Violin et al., 2010). Administration of TRV120027 to rats reduces mean arterial blood pressure, as do the nonpeptide $\mathrm{AT}_{1 \mathrm{~A}}$ receptor antagonists losartan and telmisartan, but, unlike the neutral antagonists, TRV120027 increases cardiac performance and preserves cardiac stroke volume. In a canine rapid-pacing model of heart failure, TRV120027 decreases cardiac afterload while preserving renal function, suggesting that it may have utility in heart failure treatment (Boerrigter et al., 2011, 2012). Similar improvements in cardiac performance were observed upon treatment with a related $\beta$-arrestin-biased angiotensin analog, TRV120023, in a murine model of familial dilated cardiomyopathy due to expression of a mutant tropomyosin with reduced myofilament $\mathrm{Ca}^{2+}$ sensitivity (Tarigopula et al., 2015). Another arrestin-selective $\mathrm{AT}_{1 \mathrm{~A}}$ receptor agonist, TRV027, has been studied in acute heart failure in humans, but failed to demonstrate efficacy in any of the study endpoints in a phase IIb clinical trial (Felker et al., 2015; Greenberg, 2016). Given the complexity of arrestin functions in the cardiovascular system, such a failure to translate in vitro and animal data underscores the challenges of translating arrestin-selective bias into viable human therapeutics.

Osteoporosis is another therapeutic area where selective activation of $\beta$-arrestin signaling may confer benefit. During physiologic bone remodeling, PTH stimulates bone-forming osteoblasts, increasing both osteoblast number and activity, while at the same time accelerating bone turnover by causing osteoblasts to secrete soluble factors that increase the number and activity of bone-resorbing osteoclasts (Qin et al., 2004).
Traditionally, the actions of PTH in bone have been attributed to Gs-cAMP signaling (Mohan et al., 2000), yet male mice treated with the $\beta$-arrestin-biased PTH analog, [D-Trp $\left.{ }^{12}, \mathrm{Tyr}^{34}\right]$-bPTH(7-34), exhibit paradoxical increases in bone formation rate, with greater trabecular bone volume, increased osteoblast number, and accelerated mineral apposition, but no effect on osteoclast number or markers of bone turnover (GestyPalmer et al., 2009). Functional transcriptomic analysis of microarray data from treated tissues suggests that the key to this unexpected phenotype lies in the ability of [D-Trp $\left.{ }^{12}, \mathrm{Tyr}^{34}\right]-\mathrm{PTH}(7-34)$ to expand the osteoblast pool through arrestin-dependent cell cycle regulation and anti-apoptotic signaling, while uncoupling the $\mathrm{PTH}_{1}$ receptor from Gs-cAMP-dependent activation of osteoclasts (Gesty-Palmer et al., 2013; Maudsley et al., 2015).

\section{Conclusions}

The visual $/ \beta$-arrestins, like their evolutionary cousins, the $\alpha$-arrestins, arose to exploit a conserved arrestin fold to coordinate the temporal and spatial aspects of processes related to endosome trafficking, vesicle sorting, and signaling through their capacity to bind specific cargos and localize them to defined intracellular locations. Beyond the conformational flexibility to bind a wide variety of cargo proteins, visual/ $\beta$-arrestins possess additional properties that make them uniquely suited to signal regulation. The first is the capacity to exist in different intracellular pools wherein they adopt conformations that attract and hold different cargos. The second is the ability to recognize activated GPCRs, which enables them to be responsive to extracellular stimuli. The third is that they are sufficiently abundant to sequester key pathway intermediates away from their site of activation until called to the plasma membrane. In cells, the dynamic equilibrium between large pools of inactive arrestin, held in place by low-affinity interactions with IP6, $\mathrm{Ca}^{2+}$ calmodulin and microtubules, and a small pool of active arrestin bound to high-affinity GPCR docking sites, serves to keep certain signaling pathways quiet until an extracellular stimulus is received.

The other unique feature of visual $/ \beta$-arrestins is their duality of function. Arrestin binding to GRKphosphorylated GPCRs marks the dividing line between early signaling events mediated via heterotrimeric G proteins that are terminated by arrestindependent homologous desensitization and receptor endocytosis, and the initiation of arrestin-dependent signals transmitted through scaffolding of multiprotein GPCR signalsome complexes. Although G protein signaling accounts for the short-term effects on intermediary metabolism transmitted by soluble second messengers, arrestin signaling mediates longer-term effects on processes such as cell proliferation/growth, 
survival/apoptosis, and migration/chemotaxis. In effect, $\mathrm{G}$ proteins and arrestins perform complementary roles downstream of activated GPCRs, and GRK phosphorylation/arrestin binding is the switch between signaling modes. Given their position and the center of GPCR signaling, manipulating arrestin function may be the key to developing new generations of GPCRtargeted therapeutics. Impairing arrestin activity, whether using G protein-biased orthosteric agonists or allosteric modulators, inhibiting GRKs, or targeting arrestins themselves, will enhance $G$ protein signaling, reduce tachyphylaxis, and block arrestin signaling in settings where it may be deleterious. In contrast, selective activation of arrestin-dependent signaling with arrestin-biased agonists may find application in the converse situation, in which excessive GPCR stimulation underlies a pathophysiologic process.

\section{References}

Abraham D, Podar K, Pacher M, Kubicek M, Welzel N, Hemmings BA, Dilworth SM, Mischak H, Kolch W, and Baccarini M (2000) Raf-1-associated protein phosphatase $2 \mathrm{~A}$ as a positive regulator of kinase activation. J Biol Chem $\mathbf{2 7 5}$ : $22300-22304$

Abrahamsen H, Baillie G, Ngai J, Vang T, Nika K, Ruppelt A, Mustelin T, Zaccolo M, Houslay M, and Taskén K (2004) TCR- and CD28-mediated recruitment of phosphodiesterase 4 to lipid rafts potentiates TCR signaling. J Immunol 173: $4847-4858$

Adams DG, Coffee Jr RL, Zhang H, Pelech S, Strack S, and Wadzinski BE (2005) Positive regulation of Raf1-MEK1/2-ERK1/2 signaling by protein serine/threonine phosphatase 2A holoenzymes. J Biol Chem 280:42644-42654.

Ahlquist RP (1948) A study of the adrenotropic receptors. Am J Physiol 153: $586-600$.

Ahmed MR, Zhan X, Song X, Kook S, Gurevich VV, and Gurevich EV (2011) Ubiquitin ligase parkin promotes Mdm2-arrestin interaction but inhibits arrestin ubiquitination. Biochemistry 50:3749-3763.

Ahn S, Kim J, Hara MR, Ren XR, and Lefkowitz RJ (2009) beta-Arrestin-2 mediates anti-apoptotic signaling through regulation of BAD phosphorylation. J Biol Chem 284:8855-8865.

Ahn S, Kim J, Lucaveche CL, Reedy MC, Luttrell LM, Lefkowitz RJ, and Daaka Y (2002) Src-dependent tyrosine phosphorylation regulates dynamin self-assembly and ligand-induced endocytosis of the epidermal growth factor receptor. $J$ Biol Chem 277:26642-26651.

Ahn S, Maudsley S, Luttrell LM, Lefkowitz RJ, and Daaka Y (1999) Src-mediated tyrosine phosphorylation of dynamin is required for beta2-adrenergic receptor internalization and mitogen-activated protein kinase signaling. J Biol Chem 274: 1185-1188.

Ahn S, Nelson CD, Garrison TR, Miller WE, and Lefkowitz RJ (2003) Desensitization, internalization, and signaling functions of beta-arrestins demonstrated by RNA interference. Proc Natl Acad Sci USA 100:1740-1744.

Ahn S, Shenoy SK, Wei H, and Lefkowitz RJ (2004) Differential kinetic and spatial patterns of beta-arrestin and G protein-mediated ERK activation by the angiotensin II receptor. J Biol Chem 279:35518-35525.

Alloway PG, Howard L, and Dolph PJ (2000) The formation of stable rhodopsinarrestin complexes induces apoptosis and photoreceptor cell degeneration. Neuron 28:129-138.

Alvarez CE (2008) On the origins of arrestin and rhodopsin. BMC Evol Biol 8:222. Anthony DF, Sin YY, Vadrevu S, Advant N, Day JP, Byrne AM, Lynch MJ, Milligan G, Houslay MD, and Baillie GS (2011) $\beta$-Arrestin 1 inhibits the GTPase-activating protein function of ARHGAP21, promoting activation of RhoA following angiotensin II type 1A receptor stimulation. Mol Cell Biol 31:1066-1075.

Aplin M, Christensen GL, Schneider M, Heydorn A, Gammeltoft S, Kjølbye AL, Sheikh SP, and Hansen JL (2007) Differential extracellular signal-regulated kinases 1 and 2 activation by the angiotensin type 1 receptor supports distinct phenotypes of cardiac myocytes. Basic Clin Pharmacol Toxicol 100:296-301.

Appleton KM and Luttrell LM (2013) Emergent biological properties of arrestin pathway-selective biased agonism. J Recept Signal Transduct Res 33:153-161.

Aragay AM, Mellado M, Frade JM, Martin AM, Jimenez-Sainz MC, Martinez-A C, and Mayor Jr F (1998) Monocyte chemoattractant protein-1-induced CCR2B receptor desensitization mediated by the G protein-coupled receptor kinase 2. Proc Natl Acad Sci USA 95:2985-2990.

Aubry L, Guetta D, and Klein G (2009) The arrestin fold: variations on a theme. Curr Genomics 10:133-142.

Aubry L and Klein G (2013) True arrestins and arrestin-fold proteins: a structurebased appraisal. Prog Mol Biol Transl Sci 118:21-56.

Baillie GS, Adams DR, Bhari N, Houslay TM, Vadrevu S, Meng D, Li X, Dunlop A Milligan G, Bolger GB, et al. (2007) Mapping binding sites for the PDE4D5 cAMPspecific phosphodiesterase to the $\mathrm{N}$ - and C-domains of beta-arrestin using spotimmobilized peptide arrays. Biochem J 404:71-80.
Baillie GS and Houslay MD (2005) Arrestin times for compartmentalised cAMP signalling and phosphodiesterase-4 enzymes. Curr Opin Cell Biol 17:129-134.

Balla A, Erdélyi LS, Soltész-Katona E, Balla T, Várnai P, and Hunyady L (2011) Demonstration of angiotensin II-induced Ras activation in the trans-Golgi network and endoplasmic reticulum using bioluminescence resonance energy transferbased biosensors. J Biol Chem 286:5319-5327.

Barlic J, Andrews JD, Kelvin AA, Bosinger SE, DeVries ME, Xu L, Dobransky T, Feldman RD, Ferguson SS, and Kelvin DJ (2000) Regulation of tyrosine kinase activation and granule release through beta-arrestin by CXCRI. Nat Immunol 1: 227-233.

Barnes WG, Reiter E, Violin JD, Ren XR, Milligan G, and Lefkowitz RJ (2005) betaArrestin 1 and Galphaq/11 coordinately activate RhoA and stress fiber formation following receptor stimulation. J Biol Chem 280:8041-8050.

Basher F, Fan H, Zingarelli B, Borg KT, Luttrell LM, Tempel GE, Halushka PV, and Cook JA (2008) beta-Arrestin 2: a negative regulator of inflammatory responses in polymorphonuclear leukocytes. Int J Clin Exp Med 1:32-41.

Bathgate-Siryk A, Dabul S, Pandya K, Walklett K, Rengo G, Cannavo A, De Lucia C, Liccardo D, Gao E, Leosco D, et al. (2014) Negative impact of $\beta$-arrestin- 1 on postmyocardial infarction heart failure via cardiac and adrenal-dependent neurohormonal mechanisms. Hypertension 63:404-412.

Baylor DA and Burns ME (1998) Control of rhodopsin activity in vision. Eye $12(\mathrm{Pt}$ 3b):521-525.

Beaulieu JM, Marion S, Rodriguiz RM, Medvedev IO, Sotnikova TD, Ghisi V, Wetsel WC, Lefkowitz RJ, Gainetdinov RR, and Caron MG (2008) A beta-arrestin 2 signaling complex mediates lithium action on behavior. Cell 132:125-136.

Beaulieu JM, Sotnikova TD, Marion S, Lefkowitz RJ, Gainetdinov RR, and Caron MG (2005) An Akt/beta-arrestin 2/PP2A signaling complex mediates dopaminergic neurotransmission and behavior. Cell 122:261-273.

Becuwe M, Vieira N, Lara D, Gomes-Rezende J, Soares-Cunha C, Casal M, HaguenauerTsapis R, Vincent O, Paiva S, and Léon S (2012) A molecular switch on an arrestin-like protein relays glucose signaling to transporter endocytosis. J Cell Biol 196:247-259.

Beyrière F, Sommer ME, Szczepek M, Bartl FJ, Hofmann KP, Heck M, and Ritter E (2015) Formation and decay of the arrestin rhodopsin complex in native disc membranes. J Biol Chem 290:12919-12928.

Bhandari D, Trejo J, Benovic JL, and Marchese A (2007) Arrestin-2 interacts with the ubiquitin-protein isopeptide ligase atrophin-interacting protein 4 and mediates endosomal sorting of the chemokine receptor CXCR4. J Biol Chem 282: 36971-36979.

Bhattacharya M, Anborgh PH, Babwah AV, Dale LB, Dobransky T, Benovic JL, Feldman RD, Verdi JM, Rylett RJ, and Ferguson SS (2002) Beta-arrestins regulate a Ral-GDS Ral effector pathway that mediates cytoskeletal reorganization. Nat Cell Biol 4:547-555.

Birkó Z, Swiatek M, Szájli E, Medzihradszky KF, Vijgenboom E, Penyige A, Keseru J, van Wezel GP, and Biró S (2009) Lack of A-factor production induces the expression of nutrient scavenging and stress-related proteins in Streptomyces griseus. Mol Cell Proteomics 8:2396-2403.

Bjørgo E, Moltu K, and Taskén K (2011) Phosphodiesterases as targets for modulating T-cell responses. Handb Exp Pharmacol 204:345-363.

Bjørgo E, Solheim SA, Abrahamsen H, Baillie GS, Brown KM, Berge T, Okkenhaug K, Houslay MD, and Taskén K (2010) Cross talk between phosphatidylinositol 3-kinase and cyclic AMP (cAMP)-protein kinase A signaling pathways at the level of a protein kinase B/beta-arrestin/cAMP phosphodiesterase 4 complex. Mol Cell Biol 30:1660-1672.

Boerrigter G, Lark MW, Whalen EJ, Soergel DG, Violin JD, and Burnett Jr JC (2011) Cardiorenal actions of TRV120027, a novel $\beta$-arrestin-biased ligand at the angiotensin II type I receptor, in healthy and heart failure canines: a novel therapeutic strategy for acute heart failure. Circ Heart Fail 4:770-778.

Boerrigter G, Soergel DG, Violin JD, Lark MW, and Burnett Jr JC (2012) TRV120027, a novel $\beta$-arrestin biased ligand at the angiotensin II type I receptor, unloads the heart and maintains renal function when added to furosemide in experimental heart failure. Circ Heart Fail 5:627-634.

Bohn LM, Gainetdinov RR, Lin FT, Lefkowitz RJ, and Caron MG (2000) Mu-opioid receptor desensitization by beta-arrestin-2 determines morphine tolerance but not dependence. Nature 408:720-723.

Bohn LM, Lefkowitz RJ, Gainetdinov RR, Peppel K, Caron MG, and Lin FT (1999) Enhanced morphine analgesia in mice lacking beta-arrestin 2. Science 286: $2495-2498$.

Boularan C, Scott MG, Bourougaa K, Bellal M, Esteve E, Thuret A, Benmerah A, Tramier M, Coppey-Moisan M, Labbé-Jullié C, et al. (2007) beta-Arrestin 2 oligomerization controls the Mdm2-dependent inhibition of p53. Proc Natl Acad Sci USA 104:18061-18066.

Breitman M, Kook S, Gimenez LE, Lizama BN, Palazzo MC, Gurevich EV, and Gurevich VV (2012) Silent scaffolds: inhibition OF c-Jun N-terminal kinase 3 activity in cell by dominant-negative arrestin-3 mutant. $J$ Biol Chem 287: 19653-19664.

Bruchas MR, Macey TA, Lowe JD, and Chavkin C (2006) Kappa opioid receptor activation of p38 MAPK is GRK3- and arrestin-dependent in neurons and astrocytes. J Biol Chem 281:18081-18089.

Bryja V, Gradl D, Schambony A, Arenas E, and Schulte G (2007) Beta-arrestin is a necessary component of Wnt/beta-catenin signaling in vitro and in vivo. Proc Natl Acad Sci USA 104:6690-6695.

Bryja V, Schambony A, Cajánek L, Dominguez I, Arenas E, and Schulte G (2008) Beta-arrestin and casein kinase 1/2 define distinct branches of non-canonical WNT signalling pathways. EMBO Rep 9:1244-1250.

Buhl AM, Johnson NL, Dhanasekaran N, and Johnson GL (1995) G alpha 12 and G alpha 13 stimulate Rho-dependent stress fiber formation and focal adhesion assembly. J Biol Chem 270:24631-24634.

Calebiro D, Nikolaev VO, Gagliani MC, de Filippis T, Dees C, Tacchetti C, Persani L, and Lohse MJ (2009) Persistent cAMP-signals triggered by internalized G-proteincoupled receptors. PLoS Biol 7:e1000172. 
Cannavo A, Liccardo D, and Koch WJ (2013) Targeting cardiac $\beta$-adrenergic signaling via GRK2 inhibition for heart failure therapy. Front Physiol 4:264.

Carpenter G (2000) EGF receptor transactivation mediated by the proteolytic production of EGF-like agonists. Sci STKE 2000:pe1.

Casey LM, Pistner AR, Belmonte SL, Migdalovich D, Stolpnik O, Nwakanma FE, Vorobiof G, Dunaevsky O, Matavel A, Lopes CM, et al. (2010) Small molecule disruption of $\mathrm{G}$ beta gamma signaling inhibits the progression of heart failure. Circ Res 107:532-539.

Celver J, Vishnivetskiy SA, Chavkin C, and Gurevich VV (2002) Conservation of the phosphate-sensitive elements in the arrestin family of proteins. J Biol Chem 277: 9043-9048.

Celver JP, Lowe J, Kovoor A, Gurevich VV, and Chavkin C (2001) Threonine 180 is required for G-protein-coupled receptor kinase 3- and beta-arrestin 2-mediated desensitization of the mu-opioid receptor in Xenopus oocytes. J Biol Chem 276: 4894-4900.

Charest PG and Bouvier M (2003) Palmitoylation of the V2 vasopressin receptor carboxyl tail enhances beta-arrestin recruitment leading to efficient receptor endocytosis and ERK1/2 activation. J Biol Chem 278:41541-41551.

Charest PG, Terrillon S, and Bouvier M (2005) Monitoring agonist-promoted conformational changes of beta-arrestin in living cells by intramolecular BRET EMBO Rep 6:334-340.

Chastagner P, Israël A, and Brou C (2008) AIP4/Itch regulates Notch receptor degradation in the absence of ligand. PLoS One $3: \mathrm{e} 2735$.

Chen Q, Zhuo Y, Kim M, Hanson SM, Francis DJ, Vishnivetskiy SA, Altenbach C, Klug CS, Hubbell WL, and Gurevich VV (2014) Self-association of arrestin family members. Handb Exp Pharmacol 219:205-223.

Chen W, Hu LA, Semenov MV, Yanagawa S, Kikuchi A, Lefkowitz RJ, and Miller WE (2001) $\beta$-Arrestin1 modulates lymphoid enhancer factor transcriptional activity through interaction with phosphorylated dishevelled proteins. Proc Natl Acad Sci USA 98:14889-14894.

Chen W, Ren XR, Nelson CD, Barak LS, Chen JK, Beachy PA, de Sauvage F, and Lefkowitz RJ (2004) Activity-dependent internalization of smoothened mediated by beta-arrestin 2 and GRK2. Science 306:2257-2260.

Chen W, ten Berge D, Brown J, Ahn S, Hu LA, Miller WE, Caron MG, Barak LS, Nusse R, and Lefkowitz RJ (2003) Dishevelled 2 recruits beta-arrestin 2 to mediate Wnt5A-stimulated endocytosis of Frizzled 4. Science 301:1391-1394.

Cherezov V, Rosenbaum DM, Hanson MA, Rasmussen SG, Thian FS, Kobilka TS, Choi HJ, Kuhn P, Weis WI, Kobilka BK, et al. (2007) High-resolution crystal structure of an engineered human beta2-adrenergic $\mathrm{G}$ protein-coupled receptor. Science 318:1258-1265.

Cheung R, Malik M, Ravyn V, Tomkowicz B, Ptasznik A, and Collman RG (2009) An arrestin-dependent multi-kinase signaling complex mediates MIP-1beta/CCL4 signaling and chemotaxis of primary human macrophages. J Leukoc Biol $\mathbf{8 6}$ 833-845.

Chun KS, Lao HC, Trempus CS, Okada M, and Langenbach R (2009) The prostaglandin receptor EP2 activates multiple signaling pathways and beta-arrestin1 complex formation during mouse skin papilloma development. Carcinogenesis 30 $1620-1627$

Claing A (2013) $\beta$-Arrestins: modulators of small GTPase activation and function. Prog Mol Biol Transl Sci 118:149-174.

Claing A, Chen W, Miller WE, Vitale N, Moss J, Premont RT, and Lefkowitz RJ (2001) beta-Arrestin-mediated ADP-ribosylation factor 6 activation and beta 2-adrenergic receptor endocytosis. J Biol Chem 276:42509-42513.

Cleghorn WM, Branch KM, Kook S, Arnette C, Bulus N, Zent R, Kaverina I, Gurevich EV, Weaver AM, and Gurevich VV (2015) Arrestins regulate cell spreading and motility via focal adhesion dynamics. Mol Biol Cell 26:622-635.

Coffa S, Breitman M, Hanson SM, Callaway K, Kook S, Dalby KN, and Gurevich VV (2011b) The effect of arrestin conformation on the recruitment of c-Raf1, MEK1, and ERK1/2 activation. PLoS One 6:e28723.

Coffa S, Breitman M, Spiller BW, and Gurevich VV (2011a) A single mutation in arrestin-2 prevents ERK1/2 activation by reducing c-Raf1 binding. Biochemistry 50:6951-6958.

Dabul S, Bathgate-Siryk A, Valero TR, Jafferjee M, Sturchler E, McDonald P, Koch WJ, and Lymperopoulos A (2015) Suppression of adrenal ßarrestin1-dependent aldosterone production by ARBs: head-to-head comparison. Sci Rep 5:8116.

Dasgupta P, Rastogi S, Pillai S, Ordonez-Ercan D, Morris M, Haura E, and Chellappan S (2006) Nicotine induces cell proliferation by beta-arrestinmediated activation of Src and Rb-Raf-1 pathways. J Clin Invest 116:2208-2217.

Davidson FF and Steller H (1998) Blocking apoptosis prevents blindness in Drosophila retinal degeneration mutants. Nature 391:587-591.

Davis RJ (2000) Signal transduction by the JNK group of MAP kinases. Cell 103: 239-252.

DeFea KA (2013) Arrestins in actin reorganization and cell migration. Prog Mol Biol Transl Sci 118:205-222.

DeFea KA, Vaughn ZD, O'Bryan EM, Nishijima D, Déry O, and Bunnett NW (2000a) The proliferative and antiapoptotic effects of substance $\mathrm{P}$ are facilitated by for mation of a beta -arrestin-dependent scaffolding complex. Proc Natl Acad Sci USA 97:11086-11091.

DeFea KA, Zalevsky J, Thoma MS, Déry O, Mullins RD, and Bunnett NW (2000b) beta-Arrestin-dependent endocytosis of proteinase-activated receptor 2 is required for intracellular targeting of activated ERK1/2. J Cell Biol 148:1267-1281.

De Lean A, Stadel JM, and Lefkowitz RJ (1980) A ternary complex model explains the agonist-specific binding properties of the adenylate cyclase-coupled betaadrenergic receptor. $J$ Biol Chem 255:7108-7117.

de Mendoza A, Sebé-Pedrós A, and Ruiz-Trillo I (2014) The evolution of the GPCR signaling system in eukaryotes: modularity, conservation, and the transition to metazoan multicellularity. Genome Biol Evol 6:606-619.

DeWire SM, Kim J, Whalen EJ, Ahn S, Chen M, and Lefkowitz RJ (2008) Betaarrestin-mediated signaling regulates protein synthesis. J Biol Chem 283: 10611-10620
DeWire SM, Yamashita DS, Rominger DH, Liu G, Cowan CL, Graczyk TM, Chen XT, Pitis PM, Gotchev D, Yuan C, et al. (2013) A G protein-biased ligand at the $\mu$-opioid receptor is potently analgesic with reduced gastrointestinal and respiratory dysfunction compared with morphine. J Pharmacol Exp Ther 344:708-717.

Dinculescu A, McDowell JH, Amici SA, Dugger DR, Richards N, Hargrave PA, and Smith WC (2002) Insertional mutagenesis and immunochemical analysis of visual arrestin interaction with rhodopsin. J Biol Chem 277:11703-11708,

Dolph PJ, Ranganathan R, Colley NJ, Hardy RW, Socolich M, and Zuker CS (1993) Arrestin function in inactivation of $\mathrm{G}$ protein-coupled receptor rhodopsin in vivo. Science 260:1910-1916.

Dubois L, Lecourtois M, Alexandre C, Hirst E, and Vincent JP (2001) Regulated endocytic routing modulates wingless signaling in Drosophila embryos. Cell 105: $613-624$.

Ellis J and Seidenberg M (2000) Interactions of alcuronium, TMB-8, and other allosteric ligands with muscarinic acetylcholine receptors: studies with chimeric receptors. Mol Pharmacol 58:1451-1460.

Eng AG, Kelver DA, Hedrick TP, and Swanson GT (2016) Transduction of group I mGluR-mediated synaptic plasticity by $\beta$-arrestin 2 signaling. Nat Commun 7 13571.

Fan H, Luttrell LM, Tempel GE, Senn JJ, Halushka PV, and Cook JA (2007) $\beta$-arrestins 1 and 2 differentially regulate LPS-induced signaling and proinflammatory gene expression. Mol Immunol 44:3092-3099.

Feinstein TN, Yui N, Webber MJ, Wehbi VL, Stevenson HP, King Jr JD, Hallows KR, Brown D, Bouley R, and Vilardaga JP (2013) Noncanonical control of vasopressin receptor type 2 signaling by retromer and arrestin. J Biol Chem 288:27849-27860. Felker GM, Butler J, Collins SP, Cotter G, Davison BA, Ezekowitz JA, Filippatos G, Levy PD, Metra M, Ponikowski P, et al. (2015) Heart failure therapeutics on the basis of a biased ligand of the angiotensin-2 type 1 receptor: rationale and design of the BLAST-AHF study (Biased Ligand of the Angiotensin Receptor Study in Acute Heart Failure). JACC Heart Fail 3:193-201.

Ferguson SS (2001) Evolving concepts in G protein-coupled receptor endocytosis: the role in receptor desensitization and signaling. Pharmacol Rev 53:1-24.

Ferrandon S, Feinstein TN, Castro M, Wang B, Bouley R, Potts JT, Gardella TJ, and Vilardaga JP (2009) Sustained cyclic AMP production by parathyroid hormone receptor endocytosis. Nat Chem Biol 5:734-742.

Fessart D, Simaan M, and Laporte SA (2005) c-Src regulates clathrin adapter protein 2 interaction with beta-arrestin and the angiotensin II type 1 receptor during clathrin-mediated internalization. Mol Endocrinol 19:491-503.

Fessart D, Simaan M, Zimmerman B, Comeau J, Hamdan FF, Wiseman PW, Bouvier M, and Laporte SA (2007) Src-dependent phosphorylation of beta2-adaptin dissociates the beta-arrestin-AP-2 complex. J Cell Sci 120:1723-1732.

Finger EC, Lee NY, You HJ, and Blobe GC (2008) Endocytosis of the type III transforming growth factor-beta (TGF-beta) receptor through the clathrinindependent/lipid raft pathway regulates TGF-beta signaling and receptor downregulation. J Biol Chem 283:34808-34818.

Fong AM, Premont RT, Richardson RM, Yu YR, Lefkowitz RJ, and Patel DD (2002) Defective lymphocyte chemotaxis in beta-arrestin2- and GRK6-deficient mice. Proc Natl Acad Sci USA 99:7478-7483.

Furness SGB, Liang Y-L, Nowell CJ, Halls ML, Wookey PJ, Dal Maso E, Inoue A, Christopoulos A, Wootten D, and Sexton PM (2016) Ligand-dependent modulation of G1 protein conformation alters drug efficacy. Cell 167:739-749.e11.

Gaidarov I, Krupnick JG, Falck JR, Benovic JL, and Keen JH (1999) Arrestin function in $\mathrm{G}$ protein-coupled receptor endocytosis requires phosphoinositide binding. EMBO J 18:871-881.

Galandrin S, Oligny-Longpré G, and Bouvier M (2007) The evasive nature of drug efficacy: implications for drug discovery. Trends Pharmacol Sci 28: $423-430$

Galet C and Ascoli M (2008) Arrestin-3 is essential for the activation of Fyn by the luteinizing hormone receptor (LHR) in MA-10 cells. Cell Signal 20:1822-1829.

Gallon M, Clairfeuille T, Steinberg F, Mas C, Ghai R, Sessions RB, Teasdale RD, Collins BM, and Cullen PJ (2014) A unique PDZ domain and arrestin-like fold interaction reveals mechanistic details of endocytic recycling by SNX27-retromer. Proc Natl Acad Sci USA 111:E3604-E3613.

Gao H, Sun Y, Wu Y, Luan B, Wang Y, Qu B, and Pei G (2004) Identification of betaarrestin2 as a G protein-coupled receptor-stimulated regulator of NF-kappaB pathways. Mol Cell 14:303-317.

Gao Y, Peterson S, Masri B, Hougland MT, Adham N, Gyertyán I, Kiss B, Caron MG, and El-Mallakh RS (2015) Cariprazine exerts antimanic properties and interferes with dopamine D2 receptor $\beta$-arrestin interactions. Pharmacol Res Perspect 3 e00073.

Ge L, Ly Y, Hollenberg M, and DeFea K (2003) A beta-arrestin-dependent scaffold is associated with prolonged MAPK activation in pseudopodia during proteaseactivated receptor-2-induced chemotaxis. J Biol Chem 278:34418-34426.

Ge L, Shenoy SK, Lefkowitz RJ, and DeFea K (2004) Constitutive protease-activated receptor-2-mediated migration of MDA MB-231 breast cancer cells requires both beta-arrestin-1 and -2. J Biol Chem 279:55419-55424.

Gesty-Palmer D, El Shewy H, Kohout TA, and Luttrell LM (2005) beta-Arrestin 2 expression determines the transcriptional response to lysophosphatidic acid stimulation in murine embryo fibroblasts. J Biol Chem 280:32157-32167.

Gesty-Palmer D, Flannery P, Yuan L, Corsino L, Spurney R, Lefkowitz RJ, and Luttrell LM (2009) A beta-arrestin-biased agonist of the parathyroid hormone receptor (PTH1R) promotes bone formation independent of $\mathrm{G}$ protein activation. Sci Transl Med 1:1ra1.

Gesty-Palmer D, Yuan L, Martin B, Wood 3rd WH, Lee MH, Janech MG, Tsoi LC Zheng WJ, Luttrell LM, and Maudsley S (2013) $\beta$-arrestin-selective G proteincoupled receptor agonists engender unique biological efficacy in vivo. Mol Endocrinol 27:296-314.

Ghalayini AJ, Desai N, Smith KR, Holbrook RM, Elliott MH, and Kawakatsu H (2002) Light-dependent association of Src with photoreceptor rod outer segment membrane proteins in vivo. J Biol Chem 277:1469-1476. 
Gimenez LE, Babilon S, Wanka L, Beck-Sickinger AG, and Gurevich VV (2014a) Mutations in arrestin-3 differentially affect binding to neuropeptide Y receptor subtypes. Cell Signal 26:1523-1531.

Gimenez LE, Kook S, Vishnivetskiy SA, Ahmed MR, Gurevich EV, and Gurevich VV (2012a) Role of receptor-attached phosphates in binding of visual and non-visual arrestins to G protein-coupled receptors. J Biol Chem 287:9028-9040.

Gimenez LE, Vishnivetskiy SA, Baameur F, and Gurevich VV (2012b) Manipulation of very few receptor discriminator residues greatly enhances receptor specificity of non-visual arrestins. J Biol Chem 287:29495-29505.

Gimenez LE, Vishnivetskiy SA, and Gurevich VV (2014b) Targeting individual GPCRs with redesigned nonvisual arrestins. Handb Exp Pharmacol 219:153-170.

Gloriam DE, Fredriksson R, and Schiöth HB (2007) The G protein-coupled receptor subset of the rat genome. BMC Genomics 8:338.

Godin CM, Ferreira LT, Dale LB, Gros R, Cregan SP, and Ferguson SS (2010) The small GTPase Ral couples the angiotensin II type 1 receptor to the activation of phospholipase C-delta 1. Mol Pharmacol 77:388-395.

Goel R, Phillips-Mason PJ, Raben DM, and Baldassare JJ (2002) alpha-Thrombin induces rapid and sustained Akt phosphorylation by beta-arrestin1-dependent and -independent mechanisms, and only the sustained Akt phosphorylation is essential for G1 phase progression. J Biol Chem 277:18640-18648.

Gong K, Li Z, Xu M, Du J, Lv Z, and Zhang Y (2008) A novel protein kinase A-independent, beta-arrestin-1-dependent signaling pathway for p38 mitogenactivated protein kinase activation by beta2-adrenergic receptors. $J$ Biol Chem 283:29028-29036.

Goodman Jr OB, Krupnick JG, Gurevich VV, Benovic JL, and Keen JH (1997) Arrestin/Clathrin interaction: localization of the arrestin binding locus to the clathrin terminal domain. J Biol Chem 272:15017-15022.

Goodman Jr OB, Krupnick JG, Santini F, Gurevich VV, Penn RB, Gagnon AW, Keen $\mathrm{JH}$, and Benovic JL (1996) Beta-arrestin acts as a clathrin adaptor in endocytosis of the beta2-adrenergic receptor. Nature 383:447-450.

Granzin J, Cousin A, Weirauch M, Schlesinger R, Büldt G, and Batra-Safferling R (2012) Crystal structure of p44, a constitutively active splice variant of visual arrestin. J Mol Biol 416:611-618.

Granzin J, Wilden U, Choe HW, Labahn J, Krafft B, and Büldt G (1998) X-ray crystal structure of arrestin from bovine rod outer segments. Nature 391:918-921.

Greenberg B (2016) Novel therapies in heart failure - Where do they stand? Circ $J$ 80:1882-1891.

Gullapalli S and Ramarao P (2002) Role of L-type $\mathrm{Ca}(2+)$ channels in pertussis toxin induced antagonism of $\mathrm{U} 50,488 \mathrm{H}$ analgesia and hypothermia. Brain Res 946 191-197.

Gurevich VV and Benovic JL (1993) Visual arrestin interaction with rhodopsin: sequential multisite binding ensures strict selectivity toward light-activated phosphorylated rhodopsin. J Biol Chem 268:11628-11638.

Gurevich VV and Benovic JL (1995) Visual arrestin binding to rhodopsin: diverse functional roles of positively charged residues within the phosphorylationrecognition region of arrestin. J Biol Chem 270:6010-6016.

Gurevich VV and Benovic JL (1997) Mechanism of phosphorylation-recognition by visual arrestin and the transition of arrestin into a high affinity binding state. $\mathrm{Mol}$ Pharmacol 51:161-169.

Gurevich VV, Dion SB, Onorato JJ, Ptasienski J, Kim CM, Sterne-Marr R, Hosey MM, and Benovic JL (1995) Arrestin interactions with G protein-coupled receptors: direct binding studies of wild type and mutant arrestins with rhodopsin, beta 2-adrenergic, and $\mathrm{m} 2$ muscarinic cholinergic receptors. $J$ Biol Chem 270:720-731.

Gurevich VV and Gurevich EV (2013) Structural determinants of arrestin functions. Prog Mol Biol Transl Sci 118:57-92.

Gurevich VV, Hanson SM, Song X, Vishnivetskiy SA, and Gurevich EV (2011) The functional cycle of visual arrestins in photoreceptor cells. Prog Retin Eye Res 30: $405-430$.

Gurevich VV, Pals-Rylaarsdam R, Benovic JL, Hosey MM, and Onorato JJ (1997) Agonist-receptor-arrestin, an alternative ternary complex with high agonist af finity. J Biol Chem 272:28849-28852.

Gurevich VV, Song X, Vishnivetskiy SA, and Gurevich EV (2014) Enhanced phosphorylation-independent arrestins and gene therapy. Handb Exp Pharmacol 219:133-152.

Haft CR, de la Luz Sierra M, Bafford R, Lesniak MA, Barr VA, and Taylor SI (2000) Human orthologs of yeast vacuolar protein sorting proteins Vps26, 29, and 35 assembly into multimeric complexes. Mol Biol Cell 11:4105-4116.

Haitina T, Fredriksson R, Foord SM, Schiöth HB, and Gloriam DE (2009) The G protein-coupled receptor subset of the dog genome is more similar to that in humans than rodents. $B M C$ Genomics $\mathbf{1 0} \cdot 24$

Hall RA, Premont RT, Chow CW, Blitzer JT, Pitcher JA, Claing A, Stoffel RH, Barak LS, Shenolikar S, Weinman EJ, et al. (1998) The beta2-adrenergic receptor in teracts with the $\mathrm{Na}+\mathrm{H}+-$ exchanger regulatory factor to control $\mathrm{Na}+/ \mathrm{H}+$ exchange Nature 392:626-630.

Han M, Gurevich VV, Vishnivetskiy SA, Sigler PB, and Schubert C (2001) Crystal structure of beta-arrestin at $1.9 \mathrm{~A}$ : possible mechanism of receptor binding and membrane translocation. Structure 9:869-880.

Han SO, Kommaddi RP, and Shenoy SK (2013) Distinct roles for $\beta$-arrestin2 and arrestin-domain-containing proteins in $\beta 2$ adrenergic receptor trafficking. EMBO Rep 14:164-171.

Han WD, Kawamoto S, Hosoya Y, Fujita M, Sadaie Y, Suzuki K, Ohashi Y, Kawamura F, and Ochi K (1998) A novel sporulation-control gene (spo0M) of Bacillus subtilis with a sigmaH-regulated promoter. Gene 217:31-40.

Hanson SM, Cleghorn WM, Francis DJ, Vishnivetskiy SA, Raman D, Song X, Nair KS, Slepak VZ, Klug CS, and Gurevich VV (2007a) Arrestin mobilizes signaling proteins to the cytoskeleton and redirects their activity. $\mathrm{J} \mathrm{Mol} \mathrm{Biol}$ 368:375-387.

Hanson SM, Francis DJ, Vishnivetskiy SA, Klug CS, and Gurevich VV (2006b) Visual arrestin binding to microtubules involves a distinct conformational change. J Biol Chem 281:9765-9772.
Hanson SM, Francis DJ, Vishnivetskiy SA, Kolobova EA, Hubbell WL, Klug CS, and Gurevich VV (2006a) Differential interaction of spin-labeled arrestin with inactive and active phosphorhodopsin. Proc Natl Acad Sci USA 103:4900-4905.

Hanson SM, Van Eps N, Francis DJ, Altenbach C, Vishnivetskiy SA, Arshavsky VY, Klug CS, Hubbell WL, and Gurevich VV (2007b) Structure and function of the visual arrestin oligomer. $E M B O J \mathbf{2 6 : 1 7 2 6 - 1 7 3 6 . ~}$

Hanson SM, Vishnivetskiy SA, Hubbell WL, and Gurevich VV (2008) Opposing effects of inositol hexakisphosphate on rod arrestin and arrestin2 self-association. Biochemistry 47:1070-1075.

Harding VB, Jones LR, Lefkowitz RJ, Koch WJ, and Rockman HA (2001) Cardiac $\beta$ ARK1 inhibition prolongs survival and augments $\beta$ blocker therapy in a mouse model of severe heart failure. Proc Natl Acad Sci USA 98:5809-5814.

Hirsch JA, Schubert C, Gurevich VV, and Sigler PB (1999) The 2.8 A crystal structure of visual arrestin: a model for arrestin's regulation. Cell 97:257-269.

Hoeppner CZ, Cheng N, and Ye RD (2012) Identification of a nuclear localization sequence in $\beta$-arrestin-1 and its functional implications. $J$ Biol Chem 287 $8932-8943$

Homan KT, Larimore KM, Elkins JM, Szklarz M, Knapp S, and Tesmer JJ (2015) Identification and structure-function analysis of subfamily selective $\mathrm{G}$ proteincoupled receptor kinase inhibitors. ACS Chem Biol 10:310-319.

Houndolo T, Boulay PL, and Claing A (2005) G protein-coupled receptor endocytosis in ARD-ribosylation factor 6-depleted cells. J Biol Chem 280:5598-5604.

$\mathrm{Hu} \mathrm{H}$, Columbus J, Zhang Y, Wu D, Lian L, Yang S, Goodwin J, Luczak C, Carter M, Chen L, et al. (2004) A map of WW domain family interactions. Proteomics 4: 643-655

Huang SP, Brown BM, and Craft CM (2010) Visual arrestin 1 acts as a modulator for $\mathrm{N}$-ethylmaleimide-sensitive factor in the photoreceptor synapse. $J$ Neurosci 30: 9381-9391.

Hunton DL, Barnes WG, Kim J, Ren XR, Violin JD, Reiter E, Milligan G, Patel DD, and Lefkowitz RJ (2005) Beta-arrestin 2-dependent angiotensin II type 1A receptor-mediated pathway of chemotaxis. Mol Pharmacol 67:1229-1236.

Hupfeld CJ, Resnik JL, Ugi S, and Olefsky JM (2005) Insulin-induced beta-arrestin1 Ser-412 phosphorylation is a mechanism for desensitization of ERK activation by Galphai-coupled receptors. J Biol Chem 280:1016-1023.

Hwang J, Suh HW, Jeon YH, Hwang E, Nguyen LT, Yeom J, Lee SG, Lee C, Kim KJ, Kang BS, et al. (2014) The structural basis for the negative regulation of thioredoxin by thioredoxin-interacting protein. Nat Commun 5:2958.

Imamura T, Huang J, Dalle S, Ugi S, Usui I, Luttrell LM, Miller WE, Lefkowitz RJ, and Olefsky JM (2001) beta-Arrestin-mediated recruitment of the Src family kinase Yes mediates endothelin-1-stimulated glucose transport. $J$ Biol Chem 276 43663-43667.

Jafri F, El-Shewy HM, Lee M-H, Kelly M, Luttrell DK, and Luttrell LM (2006) Constitutive ERK1/2 activation by a chimeric neurokinin 1 receptor-beta-arrestin 1 fusion protein: probing the composition and function of the $\mathrm{G}$ protein-coupled receptor "signalsome." J Biol Chem 281:19346-19357.

Jiang D, Xie T, Liang J, and Noble PW (2013) $\beta$-Arrestins in the immune system. Prog Mol Biol Transl Sci 118:359-393.

Kang DS, Kern RC, Puthenveedu MA, von Zastrow M, Williams JC, and Benovic JL (2009) Structure of an arrestin2-clathrin complex reveals a novel clathrin binding domain that modulates receptor trafficking. J Biol Chem 284:29860-29872.

Kang J, Shi Y, Xiang B, Qu B, Su W, Zhu M, Zhang M, Bao G, Wang F, Zhang X, et al (2005) A nuclear function of beta-arrestin1 in GPCR signaling: regulation of histone acetylation and gene transcription. Cell 123:833-847.

Kang Y, Zhou XE, Gao X, He Y, Liu W, Ishchenko A, Barty A, White TA, Yefanov O, Han GW, et al. (2015) Crystal structure of rhodopsin bound to arrestin by femtosecond X-ray laser. Nature 523:561-567.

Karin M and Ben-Neriah Y (2000) Phosphorylation meets ubiquitination: the control of NF-[kappa]B activity. Annu Rev Immunol 18:621-663.

Kather H, Aktories K, Schulz G, and Jakobs KH (1983) Islet-activating protein discriminates the antilipolytic mechanism of insulin from that of other antilipolytic compounds. FEBS Lett 161:149-152.

Kenakin TP (2009) '7TM receptor allostery: putting numbers to shapeshifting proteins. Trends Pharmacol Sci 30:460-469.

Kenakin TP (2012) Biased signalling and allosteric machines: new vistas and challenges for drug discovery. $\mathrm{Br}$ J Pharmacol 165:1659-1669.

Kenakin T (2015) The effective application of biased signaling to new drug discovery. Mol Pharmacol 88:1055-1061.

Kenakin T and Christopoulos A (2013) Signalling bias in new drug discovery: detection, quantification and therapeutic impact. Nat Rev Drug Discov 12:205-216.

Kenakin T and Miller LJ (2010) Seven transmembrane receptors as shapeshifting proteins: the impact of allosteric modulation and functional selectivity on new drug discovery. Pharmacol Rev 62:265-304.

Kendall RT, Lee MH, Pleasant DL, Robinson K, Kuppuswamy D, McDermott PJ, and Luttrell LM (2014) Arrestin-dependent angiotensin AT1 receptor signaling regulates Akt and mTor-mediated protein synthesis. J Biol Chem 289 26155-26166.

Kendall RT, Strungs EG, Rachidi SM, Lee MH, El-Shewy HM, Luttrell DK, Janech MG, and Luttrell LM (2011) The beta-arrestin pathway-selective type 1A angiotensin receptor (AT1A) agonist [Sar1,Ile4,Ile8]angiotensin II regulates a robust G protein-independent signaling network. J Biol Chem 286:19880-19891.

Khoury E, Nikolajev L, Simaan M, Namkung Y, and Laporte SA (2014) Differential regulation of endosomal GPCR/ $\beta$-arrestin complexes and trafficking by MAPK J Biol Chem 289:23302-23317.

Kieselbach T, Irrgang KD, and Rüppel H (1994) A segment corresponding to amino acids Val170-Arg182 of bovine arrestin is capable of binding to phosphorylated rhodopsin. Eur J Biochem 226:87-97.

Kirchberg K, Kim TY, Möller M, Skegro D, Dasara Raju G, Granzin J, Büldt G, Schlesinger R, and Alexiev U (2011) Conformational dynamics of helix 8 in the GPCR rhodopsin controls arrestin activation in the desensitization process. Proc Natl Acad Sci USA 108:18690-18695. 
Kim GH, Her JH, and Han JK (2008a) Ryk cooperates with Frizzled 7 to promote Wnt11-mediated endocytosis and is essential for Xenopus laevis convergent extension movements. J Cell Biol 182:1073-1082.

Kim J, Ahn S, Rajagopal K, and Lefkowitz RJ (2009) Independent beta-arrestin2 and $\mathrm{Gq} /$ protein kinase Czeta pathways for ERK stimulated by angiotensin type 1A receptors in vascular smooth muscle cells converge on transactivation of the epidermal growth factor receptor. J Biol Chem 284:11953-11962.

Kim J, Ahn S, Ren XR, Whalen EJ, Reiter E, Wei H, and Lefkowitz RJ (2005) Functional antagonism of different $\mathrm{G}$ protein-coupled receptor kinases for betaarrestin-mediated angiotensin II receptor signaling. Proc Natl Acad Sci USA 102: 1442-1447.

Kim J, Zhang L, Peppel K, Wu JH, Zidar DA, Brian L, DeWire SM, Exum ST, Lefkowitz RJ, and Freedman NJ (2008b) Beta-arrestins regulate atherosclerosis and neointimal hyperplasia by controlling smooth muscle cell proliferation and migration. Circ Res 103:70-79.

Kim JH, Cho EY, Min C, Park JH, and Kim KM (2008c) Characterization of functional roles of DRY motif in the 2nd intracellular loop of dopamine D2 and D3 receptors. Arch Pharm Res 31:474-481.

Kim M, Hanson SM, Vishnivetskiy SA, Song X, Cleghorn WM, Hubbell WL, and Gurevich VV (2011) Robust self-association is a common feature of mammalian visual arrestin-1. Biochemistry 50:2235-2242

Kim YJ, Hofmann KP, Ernst OP, Scheerer P, Choe HW, and Sommer ME (2013) Crystal structure of pre-activated arrestin p44. Nature 497:142-146.

Kim YM and Benovic JL (2002) Differential roles of arrestin-2 interaction with clathrin and adaptor protein 2 in G protein-coupled receptor trafficking. J Biol Chem 277:30760-30768.

Kiselev A, Socolich M, Vinós J, Hardy RW, Zuker CS, and Ranganathan R (2000) A molecular pathway for light-dependent photoreceptor apoptosis in Drosophila. Neuron 28:139-152.

Kisselev OG, Downs MA, McDowell JH, and Hargrave PA (2004a) Conformational changes in the phosphorylated C-terminal domain of rhodopsin during rhodopsin arrestin interactions. J Biol Chem 279:51203-51207.

Kisselev OG, McDowell JH, and Hargrave PA (2004b) The arrestin-bound conformation and dynamics of the phosphorylated carboxy-terminal region of rhodopsin. FEBS Lett 564:307-311.

Kook S, Zhan X, Kaoud TS, Dalby KN, Gurevich VV, and Gurevich EV (2013) Arrestin-3 binds c-Jun N-terminal kinase 1 (JNK1) and JNK2 and facilitates the activation of these ubiquitous JNK isoforms in cells via scaffolding. J Biol Chem 288:37332-37342.

Kopan R and Ilagan MX (2009) The canonical Notch signaling pathway: unfolding the activation mechanism. Cell 137:216-233.

Kotula JW, Sun J, Li M, Pratico ED, Fereshteh MP, Ahrens DP, Sullenger BA, and Kovacs JJ (2014) Targeted disruption of $\beta$-arrestin 2-mediated signaling pathways by aptamer chimeras leads to inhibition of leukemic cell growth. PLoS One 9:e93441.

Kovacs JJ, Hara MR, Davenport CL, Kim J, and Lefkowitz RJ (2009) Arrestin development: emerging roles for beta-arrestins in developmental signaling pathways. Dev Cell 17:443-458.

Kovacs JJ, Whalen EJ, Liu R, Xiao K, Kim J, Chen M, Wang J, Chen W, and Lefkowitz RJ (2008) Beta-arrestin-mediated localization of smoothened to the primary cilium. Science 320:1777-1781.

Kovoor A, Celver J, Abdryashitov RI, Chavkin C, and Gurevich VV (1999) Targeted construction of phosphorylation-independent beta-arrestin mutants with constitutive activity in cells. J Biol Chem 274:6831-6834.

Krasel C, Bünemann M, Lorenz K, and Lohse MJ (2005) $\beta$-arrestin binding to the $\beta 2$ adrenergic receptor requires both receptor phosphorylation and receptor activation. J Biol Chem 280:9528-9535.

Krueger KM, Daaka Y, Pitcher JA, and Lefkowitz RJ (1997) The role of sequestration in $\mathrm{G}$ protein-coupled receptor resensitization: regulation of beta2-adrenergic receptor dephosphorylation by vesicular acidification. J Biol Chem 272:5-8.

Krupnick JG, Goodman Jr OB, Keen JH, and Benovic JL (1997) Arrestin/Clathrin interaction: localization of the clathrin binding domain of nonvisual arrestins to the carboxy terminus. J Biol Chem 272:15011-15016.

Kuhr FK, Zhang Y, Brovkovych V, and Skidgel RA (2010) Beta-arrestin 2 is required for $\mathrm{B} 1$ receptor-dependent post-translational activation of inducible nitric oxide synthase. FASEB J 24:2475-2483.

Kyriakis JM and Avruch J (2001) Mammalian mitogen-activated protein kinase signal transduction pathways activated by stress and inflammation. Physiol Rev 81:807-869.

Kyriakis JM and Avruch J (2012) Mammalian MAPK signal transduction pathways activated by stress and inflammation: a 10-year update. Physiol Rev 92:689-737.

Land BB, Bruchas MR, Lemos JC, Xu M, Melief EJ, and Chavkin C (2008) The dysphoric component of stress is encoded by activation of the dynorphin kappaopioid system. J Neurosci 28:407-414.

Lappano R and Maggiolini M (2011) G protein-coupled receptors: novel targets for drug discovery in cancer. Nat Rev Drug Discov 10:47-60.

Laporte SA, Oakley RH, Holt JA, Barak LS, and Caron MG (2000) The interaction of beta-arrestin with the AP-2 adaptor is required for the clustering of beta 2-adrenergic receptor into clathrin-coated pits. J Biol Chem 275:23120-23126.

Laporte SA, Oakley RH, Zhang J, Holt JA, Ferguson SS, Caron MG, and Barak LS (1999) The beta2-adrenergic receptor/betaarrestin complex recruits the clathrin adaptor AP-2 during endocytosis. Proc Natl Acad Sci USA 96:3712-3717.

Lazareno S, Popham A, and Birdsall NJM (1998) Muscarinic interactions of bisindolylmaleimide analogues. Eur J Pharmacol 360:281-284

Lee M-H, Appleton KM, Strungs EG, Kwon JY, Morinelli TA, Peterson YK, Laporte SA, and Luttrell LM (2016) The conformational signature of $\beta$-arrestin2 predicts its trafficking and signalling functions. Nature 531:665-668.

Lee M-H, El-Shewy HM, Luttrell DK, and Luttrell LM (2008) Role of beta-arrestinmediated desensitization and signaling in the control of angiotensin AT1a receptor-stimulated transcription. J Biol Chem 283:2088-2097.
Lee SJ, Xu H, Kang LW, Amzel LM, and Montell C (2003) Light adaptation through phosphoinositide-regulated translocation of Drosophila visual arrestin. Neuron 39: 121-132.

Li T, Franson WK, Gordon JW, Berson EL, and Dryja TP (1995) Constitutive activation of phototransduction by K296E opsin is not a cause of photoreceptor degeneration. Proc Natl Acad Sci USA 92:3551-3555.

Li TT, Alemayehu M, Aziziyeh AI, Pape C, Pampillo M, Postovit LM, Mills GB, Babwah AV, and Bhattacharya M (2009) Beta-arrestin/Ral signaling regulates lysophosphatidic acid-mediated migration and invasion of human breast tumor cells. Mol Cancer Res 7:1064-1077.

Liggett SB (2011) Phosphorylation barcoding as a mechanism of directing GPCR signaling. Sci Signal 4:pe36.

Lima-Fernandes E, Enslen H, Camand E, Kotelevets L, Boularan C, Achour L, Benmerah A, Gibson LC, Baillie GS, Pitcher JA, et al. (2011) Distinct functiona outputs of PTEN signalling are controlled by dynamic association with $\beta$-arrestins. EMBO J 30:2557-2568.

Lin A and DeFea KA (2013) $\beta$-Arrestin-kinase scaffolds: turn them on or turn them off? Wiley Interdiscip Rev Syst Biol Med 5:231-241.

Lin FT, Chen W, Shenoy S, Cong M, Exum ST, and Lefkowitz RJ (2002) Phosphorylation of beta-arrestin2 regulates its function in internalization of beta(2)-adrenergic receptors. Biochemistry 41:10692-10699.

Lin FT, Krueger KM, Kendall HE, Daaka Y, Fredericks ZL, Pitcher JA and Lefkowitz RJ (1997) Clathrin-mediated endocytosis of the beta-adrenergic receptor is regulated by phosphorylation/dephosphorylation of beta-arrestin 1 . $J$ Biol Chem 272:31051-31057.

Lin FT, Miller WE, Luttrell LM, and Lefkowitz RJ (1999) Feedback regulation of beta-arrestin 1 function by extracellular signal-regulated kinases. J Biol Chem 274: 15971-15974.

Lodeiro M, Alén BO, Mosteiro CS, Beiroa D, Nogueiras R, Theodoropoulou M, Pardo M, Gallego R, Pazos Y, Casanueva FF, et al. (2011) The SHP-1 protein tyrosine phosphatase negatively modulates Akt signaling in the ghrelin/GHSR1a system. Mol Biol Cell 22:4182-4191.

Luan B, Zhang Z, Wu Y, Kang J, and Pei G (2005) Beta-arrestin2 functions as a phosphorylation-regulated suppressor of UV-induced NF-kappaB activation. EMBO J 24:4237-4246.

Luttrell LM (2013) Arrestin pathways as drug targets. Prog Mol Biol Transl Sci 118 469-497.

Luttrell LM (2014) Minireview: more than just a hammer: ligand "bias" and pharmaceutical discovery. Mol Endocrinol 28:281-294.

Luttrell LM, Ferguson SS, Daaka Y, Miller WE, Maudsley S, Della Rocca GJ, Lin F, Kawakatsu H, Owada K, Luttrell DK, et al. (1999) Beta-arrestin-dependent formation of beta2 adrenergic receptor-Src protein kinase complexes. Science $\mathbf{2 8 3}$ : $655-661$.

Luttrell LM and Gesty-Palmer D (2010) Beyond desensitization: physiological relevance of arrestin-dependent signaling. Pharmacol Rev 62:305-330.

Luttrell LM and Kenakin TP (2011) Refining efficacy: allosterism and bias in G protein-coupled receptor signaling. Methods Mol Biol 756:3-35.

Luttrell LM, Maudsley S, and Bohn LM (2015) Fulfilling the promise of "biased" G protein-coupled receptor agonism. Mol Pharmacol 88:579-588.

Luttrell LM and Miller WE (2013) Arrestins as regulators of kinases and phosphatases. Prog Mol Biol Transl Sci 118:115-147.

Luttrell LM, Roudabush FL, Choy EW, Miller WE, Field ME, Pierce KL, and Lefkowitz RJ (2001) Activation and targeting of extracellular signal-regulated kinases by beta-arrestin scaffolds. Proc Natl Acad Sci USA 98:2449-2454.

Lymperopoulos A, Rengo G, Zincarelli C, Kim J, Soltys S, and Koch WJ (2009) An adrenal beta-arrestin 1-mediated signaling pathway underlies angiotensin II-induced aldosterone production in vitro and in vivo. Proc Natl Acad Sci USA 106:5825-5830.

Macia E, Partisani M, Paleotti O, Luton F, and Franco M (2012) Arf6 negatively controls the rapid recycling of the $\beta 2$ adrenergic receptor. J Cell Sci 125: 4026-4035.

Mahon MJ, Donowitz M, Yun CC, and Segre GV (2002) Na(+)/H(+ ) exchanger regulatory factor 2 directs parathyroid hormone 1 receptor signalling. Nature 417:858-861.

Manglik A and Kobilka B (2014) The role of protein dynamics in GPCR function: insights from the $\beta 2 A R$ and rhodopsin. Curr Opin Cell Biol 27:136-143.

Masri B, Salahpour A, Didriksen M, Ghisi V, Beaulieu JM, Gainetdinov RR, and Caron MG (2008) Antagonism of dopamine D2 receptor/beta-arrestin 2 interaction is a common property of clinically effective antipsychotics. Proc Natl Acad Sci USA 105:13656-13661.

Maudsley S, Martin B, Gesty-Palmer D, Cheung H, Johnson C, Patel S, Becker KG Wood 3rd WH, Zhang Y, Lehrmann E, et al. (2015) Delineation of a conserved arrestin-biased signaling repertoire in vivo. Mol Pharmacol 87:706-717.

McDonald PH, Chow CW, Miller WE, Laporte SA, Field ME, Lin FT, Davis RJ, and Lefkowitz RJ (2000) Beta-arrestin 2. a receptor-regulated MAPK scaffold for the activation of JNK3. Science 290:1574-1577.

McDonald PH, Cote NL, Lin FT, Premont RT, Pitcher JA, and Lefkowitz RJ (1999) Identification of NSF as a beta-arrestin1-binding protein: implications for beta2adrenergic receptor regulation. $J$ Biol Chem 274:10677-10680.

McLaughlin NJ, Banerjee A, Kelher MR, Gamboni-Robertson F, Hamiel C, Sheppard FR, Moore EE, and Silliman CC (2006) Platelet-activating factor-induced clathrinmediated endocytosis requires beta-arrestin-1 recruitment and activation of the p38 MAPK signalosome at the plasma membrane for actin bundle formation. Immunol 176:7039-7050.

Meng D, Lynch MJ, Huston E, Beyermann M, Eichhorst J, Adams DR, Klussmann E, Houslay MD, and Baillie GS (2009) MEK1 binds directly to betaarrestin1, influencing both its phosphorylation by ERK and the timing of its isoprenalinestimulated internalization. $J$ Biol Chem 284:11425-11435.

Michel MC, Brunner HR, Foster C, and Huo Y (2016) Angiotensin II type 1 receptor antagonists in animal models of vascular, cardiac, metabolic and renal disease. Pharmacol Ther 164:1-81. 
Milano SK, Kim YM, Stefano FP, Benovic JL, and Brenner C (2006) Nonvisual arrestin oligomerization and cellular localization are regulated by inositol hexakisphosphate binding. J Biol Chem 281:9812-9823.

Milano SK, Pace HC, Kim YM, Brenner C, and Benovic JL (2002) Scaffolding functions of arrestin-2 revealed by crystal structure and mutagenesis. Biochemistry 41 $3321-3328$

Miller WE, Maudsley S, Ahn S, Khan KD, Luttrell LM, and Lefkowitz RJ (2000) betaArrestin 1 interacts with the catalytic domain of the tyrosine kinase c-SRC: role of beta-arrestin1-dependent targeting of c-SRC in receptor endocytosis. J Biol Chem 275:11312-11319.

Miller WE, McDonald PH, Cai SF, Field ME, Davis RJ, and Lefkowitz RJ (2001) Identification of a motif in the carboxyl terminus of beta-arrestin2 responsible for activation of JNK3. J Biol Chem 276:27770-27777.

Miura S, Zhang J, Matsuo Y, Saku K, and Karnik SS (2004) Activation of extracellular signal-activated kinase by angiotensin II-induced Gq-independent epidermal growth factor receptor transactivation. Hypertens Res 27:765-770.

Mo W, Zhang L, Yang G, Zhai J, Hu Z, Chen Y, Chen X, Hui L, Huang R, and Hu G (2008) Nuclear beta-arrestin1 functions as a scaffold for the dephosphorylation of STAT1 and moderates the antiviral activity of IFN-gamma. Mol Cell 31:695-707.

Moaven H, Koike Y, Jao CC, Gurevich VV, Langen R, and Chen J (2013) Visual arrestin interaction with clathrin adaptor AP-2 regulates photoreceptor survival in the vertebrate retina. Proc Natl Acad Sci USA 110:9463-9468.

Mohan S, Kutilek S, Zhang C, Shen HG, Kodama Y, Srivastava AK, Wergedal JE, Beamer WG, and Baylink DJ (2000) Comparison of bone formation responses to parathyroid hormone(1-34), (1-31), and (2-34) in mice. Bone 27:471-478.

Mokarzel-Falcón L, Padrón-García JA, Carrasco-Velar R, Berry C, and MonteroCabrera LA (2008) In silico study of the human rhodopsin and meta rhodopsin II/Sarrestin complexes: impact of single point mutations related to retina degenerative diseases. Proteins 70:1133-1141.

Moussa O, Ashton AW, Fraig M, Garrett-Mayer E, Ghoneim MA, Halushka PV, and Watson DK (2008) Novel role of thromboxane receptors beta isoform in bladder cancer pathogenesis. Cancer Res 68:4097-4104.

Mukherjee A, Veraksa A, Bauer A, Rosse C, Camonis J, and Artavanis-Tsakonas S (2005) Regulation of Notch signalling by non-visual beta-arrestin. Nat Cell Biol 7: 1191-1201.

Mukherjee S, Gurevich VV, Jones JC, Casanova JE, Frank SR, Maizels ET, Bader MF, Kahn RA, Palczewski K, Aktories K, et al. (2000) The ADP ribosylation factor nucleotide exchange factor ARNO promotes beta-arrestin release necessary for luteinizing hormone/choriogonadotropin receptor desensitization. Proc Natl Acad Sci USA 97:5901-5906.

Mullershausen F, Zecri F, Cetin C, Billich A, Guerini D, and Seuwen K (2009) Persistent signaling induced by FTY720-phosphate is mediated by internalized S1P1 receptors. Nat Chem Biol 5:428-434.

Mythreye K and Blobe GC (2009) The type III TGF-beta receptor regulates epithelial and cancer cell migration through beta-arrestin2-mediated activation of Cdc42. Proc Natl Acad Sci USA 106:8221-8226.

Nabhan JF, Pan H, and Lu Q (2010) Arrestin domain-containing protein 3 recruits the NEDD4 E3 ligase to mediate ubiquitination of the beta2-adrenergic receptor. EMBO Rep 11:605-611.

Nair KS, Hanson SM, Kennedy MJ, Hurley JB, Gurevich VV, and Slepak VZ (2004) Direct binding of visual arrestin to microtubules determines the differential subcellular localization of its splice variants in rod photoreceptors. J Biol Chem $\mathbf{2 7 9}$ $41240-41248$.

Nair KS, Hanson SM, Mendez A, Gurevich EV, Kennedy MJ, Shestopalov VI, Vishnivetskiy SA, Chen J, Hurley JB, Gurevich VV, et al. (2005) Light-dependent redistribution of arrestin in vertebrate rods is an energy-independent process governed by protein-protein interactions. Neuron 46:555-567.

Nelson CD, Kovacs JJ, Nobles KN, Whalen EJ, and Lefkowitz RJ (2008) Betaarrestin scaffolding of phosphatidylinositol 4-phosphate 5-kinase Ialpha promotes agonist-stimulated sequestration of the beta2-adrenergic receptor. $\mathrm{J}$ Biol Chem 283:21093-21101.

Nelson CD, Perry SJ, Regier DS, Prescott SM, Topham MK, and Lefkowitz RJ (2007) Targeting of diacylglycerol degradation to M1 muscarinic receptors by betaarrestins. Science 315:663-666.

Nichols HL, Saffeddine M, Theriot BS, Hegde A, Polley D, El-Mays T, Vliagoftis H, Hollenberg MD, Wilson EH, Walker JK, et al. (2012) $\beta$-Arrestin-2 mediates the proinflammatory effects of proteinase-activated receptor-2 in the airway. Proc Natl Acad Sci USA 109:16660-16665.

Nobles KN, Xiao K, Ahn S, Shukla AK, Lam CM, Rajagopal S, Strachan RT, Huang TY, Bressler EA, Hara MR, et al. (2011) Distinct phosphorylation sites on the $\beta(2)$ adrenergic receptor establish a barcode that encodes differential functions of $\beta$-arrestin. Sci Signal 4:ra51.

Noma T, Lemaire A, Naga Prasad SV, Barki-Harrington L, Tilley DG, Chen J, Le Corvoisier P, Violin JD, Wei H, Lefkowitz RJ, et al. (2007) Beta-arrestin-mediated beta1-adrenergic receptor transactivation of the EGFR confers cardioprotection. $J$ Clin Invest 117:2445-2458.

Nordström K, Wallén R, Seymour J, and Nilsson D (2003) A simple visual system without neurons in jellyfish larvae. Proc Biol Sci 270:2349-2354.

Nuber S, Zabel U, Lorenz K, Nuber A, Milligan G, Tobin AB, Lohse MJ, and Hoffmann C (2016) $\beta$-Arrestin biosensors reveal a rapid, receptor-dependent activation/deactivation cycle. Nature 531:661-664

Oakley RH, Laporte SA, Holt JA, Barak LS, and Caron MG (2001) Molecular determinants underlying the formation of stable intracellular $\mathrm{G}$ protein-coupled receptor-beta-arrestin complexes after receptor endocytosis. $J$ Biol Chem 276: 19452-19460.

Oakley RH, Laporte SA, Holt JA, Caron MG, and Barak LS (2000) Differential affinities of visual arrestin, beta arrestin1, and beta arrestin2 for $\mathrm{G}$ protein-coupled receptors delineate two major classes of receptors. J Biol Chem 275:17201-17210

Oppenheimer J and Nelson HS (2008) Safety of long-acting beta-agonists in asthma: a review. Curr Opin Pulm Med 14:64-69.
Ozawa K, Whalen EJ, Nelson CD, Mu Y, Hess DT, Lefkowitz RJ, and Stamler JS (2008) S-nitrosylation of beta-arrestin regulates beta-adrenergic receptor trafficking. Mol Cell 31:395-405.

Palczewski K, Pulvermüller A, Buczylko J, Gutmann C, and Hofmann KP (1991a) Binding of inositol phosphates to arrestin. FEBS Lett 295:195-199.

Palczewski K, Pulvermüller A, Buczyłko J, and Hofmann KP (1991b) Phosphorylated rhodopsin and heparin induce similar conformational changes in arrestin. $J$ Biol Chem 266:18649-18654

Paleotti O, Macia E, Luton F, Klein S, Partisani M, Chardin P, Kirchhausen T, and Franco M (2005) The small G-protein Arf6GTP recruits the AP-2 adaptor complex to membranes. $J$ Biol Chem 280*21661-21666.

Parent JL, Labrecque P, Orsini MJ, and Benovic JL (1999) Internalization of the TXA2 receptor alpha and beta isoforms: role of the differentially spliced cooh terminus in agonist-promoted receptor internalization. J Biol Chem 274:8941-8948.

Patwari P, Chutkow WA, Cummings K, Verstraeten VL, Lammerding J, Schreiter ER, and Lee RT (2009) Thioredoxin-independent regulation of metabolism by the alpha-arrestin proteins. J Biol Chem 284:24996-25003.

Pearson G, Robinson F, Beers Gibson T, Xu BE, Karandikar M, Berman K, and Cobb MH (2001) Mitogen-activated protein (MAP) kinase pathways: regulation and physiological functions. Endocr Rev 22:153-183.

Penela P, Elorza A, Sarnago S, and Mayor Jr F (2001) Beta-arrestin- and c-Srcdependent degradation of G-protein-coupled receptor kinase 2. EMBO J 20: $5129-5138$.

Perroy J, Pontier S, Charest PG, Aubry M, and Bouvier M (2004) Real-time monitoring of ubiquitination in living cells by BRET. Nat Methods 1:203-208.

Perry SJ, Baillie GS, Kohout TA, McPhee I, Magiera MM, Ang KL, Miller WE, McLean AJ, Conti M, Houslay MD, et al. (2002) Targeting of cyclic AMP degradation to beta 2-adrenergic receptors by beta-arrestins. Science 298:834-836.

Piao L, Fang YH, Parikh KS, Ryan JJ, D’Souza KM, Theccanat T, Toth PT, Pogoriler J, Paul J, Blaxall BC, et al. (2012) GRK2-mediated inhibition of adrenergic and dopaminergic signaling in right ventricular hypertrophy: therapeutic implications in pulmonary hypertension. Circulation 126:2859-2869.

Pike NB (2005) Flushing out the role of GPR109A (HM74A) in the clinical efficacy of nicotinic acid. J Clin Invest 115:3400-3403.

Pitcher JA, Payne ES, Csortos C, DePaoli-Roach AA, and Lefkowitz RJ (1995) The G-protein-coupled receptor phosphatase: a protein phosphatase type $2 \mathrm{~A}$ with a distinct subcellular distribution and substrate specificity. Proc Natl Acad Sci USA 92:8343-8347.

Polekhina G, Ascher DB, Kok SF, Beckham S, Wilce M, and Waltham M (2013) Structure of the N-terminal domain of human thioredoxin-interacting protein. Acta Crystallogr D Biol Crystallogr 69:333-344.

Poupart ME, Fessart D, Cotton M, Laporte SA, and Claing A (2007) ARF6 regulates angiotensin II type 1 receptor endocytosis by controlling the recruitment of AP-2 and clathrin. Cell Signal 19:2370-2378.

Puca L, Chastagner P, Meas-Yedid V, Israël A, and Brou C (2013) A-arrestin 1 (ARRDC1) and $\beta$-arrestins cooperate to mediate Notch degradation in mammals. $J$ Cell Sci 126:4457-4468.

Qin L, Raggatt LJ, and Partridge NC (2004) Parathyroid hormone: a double-edged sword for bone metabolism. Trends Endocrinol Metab 15:60-65.

Qin R, Li K, Qi X, Zhou X, Wang L, Zhang P, and Zou L (2014) $\beta$-Arrestin1 promotes the progression of chronic myeloid leukaemia by regulating BCR/ABL H4 acetylation. $\mathrm{Br} J$ Cancer 111:568-576.

Raake PW, Schlegel P, Ksienzyk J, Reinkober J, Barthelmes J, Schinkel S, Pleger S, Mier W, Haberkorn U, Koch WJ, et al. (2013) AAV6.ßARKct cardiac gene therapy ameliorates cardiac function and normalizes the catecholaminergic axis in a clinically relevant large animal heart failure model. Eur Heart $J$ 34 1437-1447.

Raehal KM and Bohn LM (2011) The role of beta-arrestin2 in the severity of antinociceptive tolerance and physical dependence induced by different opioid pain therapeutics. Neuropharmacology 60:58-65.

Raehal KM, Schmid CL, Groer CE, and Bohn LM (2011) Functional selectivity at the $\mu$-opioid receptor: implications for understanding opioid analgesia and tolerance. Pharmacol Rev 63:1001-1019.

Raehal KM, Walker JK, and Bohn LM (2005) Morphine side effects in beta-arrestin 2 knockout mice. J Pharmacol Exp Ther 314:1195-1201.

Raghu P, Usher K, Jonas S, Chyb S, Polyanovsky A, and Hardie RC (2000) Constitutive activity of the light-sensitive channels TRP and TRPL in the Drosophila diacylglycerol kinase mutant, rdgA. Neuron 26:169-179.

Raghuwanshi SK, Nasser MW, Chen X, Strieter RM, and Richardson RM (2008) Depletion of beta-arrestin-2 promotes tumor growth and angiogenesis in a murine model of lung cancer. J Immunol 180:5699-5706.

Rajagopal K, Whalen EJ, Violin JD, Stiber JA, Rosenberg PB, Premont RT, Coffman TM, Rockman HA, and Lefkowitz RJ (2006) Beta-arrestin2-mediated inotropic effects of the angiotensin II type 1A receptor in isolated cardiac myocytes. Proc Natl Acad Sci USA 103:16284-16289.

Ramachandran R, Noorbakhsh F, Defea K, and Hollenberg MD (2012) Targeting proteinase-activated receptors: therapeutic potential and challenges. Nat Rev Drug Discov 11:69-86.

Rasmussen SG, Choi HJ, Fung JJ, Pardon E, Casarosa P, Chae PS, Devree BT, Rosenbaum DM, Thian FS, Kobilka TS, et al. (2011a) Structure of a nanobodystabilized active state of the $\beta(2)$ adrenoceptor. Nature 469:175-180.

Rasmussen SG, Choi HJ, Rosenbaum DM, Kobilka TS, Thian FS, Edwards PC, Burghammer M, Ratnala VR, Sanishvili R, Fischetti RF, et al. (2007) Crystal structure of the human beta2 adrenergic G-protein-coupled receptor. Nature $\mathbf{4 5 0}$ 383-387.

Rasmussen SG, DeVree BT, Zou Y, Kruse AC, Chung KY, Kobilka TS, Thian FS, Chae PS, Pardon E, Calinski D, et al. (2011b) Crystal structure of the $\beta 2$ adrenergic receptor-Gs protein complex. Nature 477:549-555.

Redila VA and Chavkin C (2008) Stress-induced reinstatement of cocaine seeking is mediated by the kappa opioid system. Psychopharmacology 200:59-70. 
Ren XR, Reiter E, Ahn S, Kim J, Chen W, and Lefkowitz RJ (2005) Different G protein-coupled receptor kinases govern $\mathrm{G}$ protein and beta-arrestin-mediated signaling of V2 vasopressin receptor. Proc Natl Acad Sci USA 102:1448-1453.

Rizo J and Südhof TC (2002) Snares and Munc18 in synaptic vesicle fusion. Nat Rev Neurosci 3:641-653.

Rockman HA, Chien KR, Choi DJ, Iaccarino G, Hunter JJ, Ross Jr J, Lefkowitz RJ, and Koch WJ (1998) Expression of a beta-adrenergic receptor kinase 1 inhibitor prevents the development of myocardial failure in gene-targeted mice. Proc Natl Acad Sci USA 95:7000-7005.

Rosanò L, Cianfrocca R, Masi S, Spinella F, Di Castro V, Biroccio A, Salvati E, Nicotra MR, Natali PG, and Bagnato A (2009) Beta-arrestin links endothelin A receptor to beta-catenin signaling to induce ovarian cancer cell invasion and metastasis. Proc Natl Acad Sci USA 106:2806-2811.

Rovati GE, Capra V, and Neubig RR (2007) The highly conserved DRY motif of class A G protein-coupled receptors: beyond the ground state. Mol Pharmacol 71: 959-964.

Sachpatzidis A, Benton BK, Manfredi JP, Wang H, Hamilton A, Dohlman HG, and Lolis E (2003) Identification of allosteric peptide agonists of CXCR4. J Biol Chem 278:896-907.

Samama P, Cotecchia S, Costa T, and Lefkowitz RJ (1993) A mutation-induced activated state of the beta 2-adrenergic receptor: extending the ternary complex model. J Biol Chem 268:4625-4636.

Sato PY, Chuprun JK, Schwartz M, and Koch WJ (2015) The evolving impact of G protein-coupled receptor kinases in cardiac health and disease. Physiol Rev 95: 377-404

Schleicher A, Kühn H, and Hofmann KP (1989) Kinetics, binding constant, and activation energy of the 48 -kDa protein-rhodopsin complex by extra-metarhodopsin II. Biochemistry 28:1770-1775.

Schmid CL, Streicher JM, Groer CE, Munro TA, Zhou L, and Bohn LM (2013) Functional selectivity of $6^{\prime}$-guanidinonaltrindole $\left(6^{\prime}\right.$-GNTI) at $\kappa$-opioid receptors in striatal neurons. J Biol Chem 288:22387-22398.

Schmid EM, Ford MG, Burtey A, Praefcke GJ, Peak-Chew SY, Mills IG, Benmerah A and McMahon HT (2006) Role of the AP2 beta-appendage hub in recruiting partners for clathrin-coated vesicle assembly. PLoS Biol 4:e262.

Schöneberg T, Schulz A, Biebermann H, Hermsdorf T, Römpler H, and Sangkuhl K (2004) Mutant G-protein-coupled receptors as a cause of human diseases. Pharmacol Ther 104:173-206.

Schröder K, Pulvermüller A, and Hofmann KP (2002) Arrestin and its splice variant Arr1-370A (p44): mechanism and biological role of their interaction with rhodopsin. $J$ Biol Chem 277:43987-43996.

Schubert C, Hirsch JA, Gurevich VV, Engelman DM, Sigler PB, and Fleming KG (1999) Visual arrestin activity may be regulated by self-association. J Biol Chem 274:21186-21190.

Scott MG, Le Rouzic E, Périanin A, Pierotti V, Enslen H, Benichou S, Marullo S, and Benmerah A (2002) Differential nucleocytoplasmic shuttling of beta-arrestins: characterization of a leucine-rich nuclear export signal in beta-arrestin2. $J$ Bio Chem 277:37693-37701.

Scott MG, Pierotti V, Storez H, Lindberg E, Thuret A, Muntaner O, Labbé-Jullié C Pitcher JA, and Marullo S (2006) Cooperative regulation of extracellular signalregulated kinase activation and cell shape change by filamin A and beta-arrestins. Mol Cell Biol 26:3432-3445.

Seachrist JL and Ferguson SS (2003) Regulation of G protein-coupled receptor endocytosis and trafficking by Rab GTPases. Life Sci 74:225-235.

Seaman MN (2004) Cargo-selective endosomal sorting for retrieval to the Golgi requires retromer. J Cell Biol 165:111-122

Semple G, Skinner PJ, Gharbaoui T, Shin YJ, Jung JK, Cherrier MC, Webb PJ, Tamura SY, Boatman PD, Sage CR, et al. (2008) 3-(1H-tetrazol-5-yl)-1,4,5,6 tetrahydro-cyclopentapyrazole (MK-0354): a partial agonist of the nicotinic acid receptor, G-protein coupled receptor $109 \mathrm{a}$, with antilipolytic but no vasodilatory activity in mice. J Med Chem 51:5101-5108.

Seo J, Tsakem EL, Breitman M, and Gurevich VV (2011) Identification of arrestin-3specific residues necessary for JNK3 kinase activation. $J$ Biol Chem 286 27894-27901.

Seta K, Nanamori M, Modrall JG, Neubig RR, and Sadoshima J (2002) AT1 receptor mutant lacking heterotrimeric G protein coupling activates the Src-Ras-ERK pathway without nuclear translocation of ERKs. J Biol Chem 277:9268-9277.

Shenoy SK, Barak LS, Xiao K, Ahn S, Berthouze M, Shukla AK, Luttrell LM, and Lefkowitz RJ (2007) Ubiquitination of beta-arrestin links seventransmembrane receptor endocytosis and ERK activation. $J$ Biol Chem 282 29549-29562.

Shenoy SK and Lefkowitz RJ (2003) Trafficking patterns of beta-arrestin and G protein-coupled receptors determined by the kinetics of beta-arrestin deubiquitination. J Biol Chem 278:14498-14506.

Shenoy SK and Lefkowitz RJ (2005) Receptor-specific ubiquitination of beta-arrestin directs assembly and targeting of seven-transmembrane receptor signalosomes. $J$ Biol Chem 280:15315-15324.

Shenoy SK, McDonald PH, Kohout TA, and Lefkowitz RJ (2001) Regulation of receptor fate by ubiquitination of activated $\beta 2$-adrenergic receptor and $\beta$-arrestin. Science 294:1307-1313.

Shenoy SK, Modi AS, Shukla AK, Xiao K, Berthouze M, Ahn S, Wilkinson KD, Miller WE, and Lefkowitz RJ (2009) Beta-arrestin-dependent signaling and trafficking of 7-transmembrane receptors is reciprocally regulated by the deubiquitinase USP33 and the E3 ligase Mdm2. Proc Natl Acad Sci USA 106:6650-6655.

Shenoy SK, Xiao K, Venkataramanan V, Snyder PM, Freedman NJ, and Weissman AM (2008) Nedd4 mediates agonist-dependent ubiquitination, lysosomal tar geting, and degradation of the beta2-adrenergic receptor. $J$ Biol Chem 283 $22166-22176$.

Shi Y, Feng Y, Kang J, Liu C, Li Z, Li D, Cao W, Qiu J, Guo Z, Bi E, et al. (2007) Critical regulation of CD4+ T cell survival and autoimmunity by beta-arrestin 1 . Nat Immunol 8:817-824.
Shi H, Rojas R, Bonifacino JS, and Hurley JH (2006) The retromer subunit Vps26 has an arrestin fold and binds Vps35 through its C-terminal domain. Nat Struct Mol Biol 13:540-548.

Shukla AK, Manglik A, Kruse AC, Xiao K, Reis RI, Tseng WC, Staus DP, Hilger D, Uysal S, Huang LY, et al. (2013) Structure of active $\beta$-arrestin-1 bound to a G-protein-coupled receptor phosphopeptide. Nature 497:137-141.

Shukla AK, Violin JD, Whalen EJ, Gesty-Palmer D, Shenoy SK, and Lefkowitz RJ (2008) Distinct conformational changes in beta-arrestin report biased agonism at seven-transmembrane receptors. Proc Natl Acad Sci USA 105:9988-9993.

Shukla AK, Westfield GH, Xiao K, Reis RI, Huang LY, Tripathi-Shukla P, Qian J, Li S, Blanc A, Oleskie AN, et al. (2014) Visualization of arrestin recruitment by a G-protein-coupled receptor. Nature 512:218-222.

Silverstein AM, Barrow CA, Davis AJ, and Mumby MC (2002) Actions of PP2A on the MAP kinase pathway and apoptosis are mediated by distinct regulatory subunits. Proc Natl Acad Sci USA 99:4221-4226.

Simonin A and Fuster D (2010) Nedd4-1 and beta-arrestin-1 are key regulators of $\mathrm{Na}+/ \mathrm{H}+$ exchanger 1 ubiquitylation, endocytosis, and function. $J$ Biol Chem 285 : 38293-38303.

Soergel DG, Subach RA, Burnham N, Lark MW, James IE, Sadler BM, Skobieranda F, Violin JD, and Webster LR (2014) Biased agonism of the $\mu$-opioid receptor by TRV130 increases analgesia and reduces on-target adverse effects versus morphine: a randomized, double-blind, placebo-controlled, crossover study in healthy volunteers. Pain 155:1829-1835.

Sommer ME, Farrens DL, McDowell JH, Weber LA, and Smith WC (2007) Dynamics of arrestin-rhodopsin interactions: loop movement is involved in arrestin activation and receptor binding. J Biol Chem 282:25560-25568.

Song X, Coffa S, Fu H, and Gurevich VV (2009a) How does arrestin assemble MAPKs into a signaling complex? J Biol Chem 284:685-695.

Song X, Gurevich EV, and Gurevich VV (2007) Cone arrestin binding to JNK3 and Mdm2: conformational preference and localization of interaction sites. J Neurochem 103:1053-1062.

Song X, Raman D, Gurevich EV, Vishnivetskiy SA, and Gurevich VV (2006) Visual and both non-visual arrestins in their "inactive" conformation bind JNK3 and Mdm2 and relocalize them from the nucleus to the cytoplasm. J Biol Chem 281: 21491-21499.

Song X, Seo J, Baameur F, Vishnivetskiy SA, Chen Q, Kook S, Kim M, Brooks EK, Altenbach C, Hong Y, et al. (2013) Rapid degeneration of rod photoreceptors expressing self-association-deficient arrestin-1 mutant. Cell Signal 25:2613-2624

Song X, Vishnivetskiy SA, Gross OP, Emelianoff K, Mendez A, Chen J, Gurevich EV, Burns ME, and Gurevich VV (2009b) Enhanced arrestin facilitates recovery and protects rods lacking rhodopsin phosphorylation. Curr Biol 19:700-705.

Spiegel AM (1996) Mutations in G proteins and G protein-coupled receptors in en docrine disease. J Clin Endocrinol Metab 81:2434-2442.

Spindel ON, Burke RM, Yan C, and Berk BC (2014) Thioredoxin-interacting protein is a biomechanical regulator of Src activity: key role in endothelial cell stress fiber formation. Circ Res 114:1125-1132.

Stambouli N, Dridi M, Wei NN, Jlizi A, Bouraoui A, and Elgaaied AB (2014) Structural insight into the binding complex: $\beta$-arrestin/CCR5 complex. J Biomol Struct Dyn 32:866-875.

Stoffel 3rd RH, Pitcher JA, and Lefkowitz RJ (1997) Targeting G protein-coupled receptor kinases to their receptor substrates. J Membr Biol 157:1-8.

Storez H, Scott MG, Issafras H, Burtey A, Benmerah A, Muntaner O, Piolot T, Tramier M, Coppey-Moisan M, Bouvier M, et al. (2005) Homo- and heterooligomerization of beta-arrestins in living cells. J Biol Chem 280:40210-40215.

Su Y, Raghuwanshi SK, Yu Y, Nanney LB, Richardson RM, and Richmond A (2005) Altered CXCR2 signaling in beta-arrestin-2-deficient mouse models. J Immuno 175:5396-5402

Sun Y, Cheng Z, Ma L, and Pei G (2002) Beta-arrestin2 is critically involved in CXCR4-mediated chemotaxis, and this is mediated by its enhancement of p38 MAPK activation. J Biol Chem 277:49212-49219.

Sutton RB, Vishnivetskiy SA, Robert J, Hanson SM, Raman D, Knox BE, Kono M, Navarro J, and Gurevich VV (2005) Crystal structure of cone arrestin at 2.3A evolution of receptor specificity. J Mol Biol 354:1069-1080.

Szczepek M, Beyrière F, Hofmann KP, Elgeti M, Kazmin R, Rose A, Bartl FJ, von Stetten D, Heck M, Sommer ME, et al. (2014) Crystal structure of a common GPCR-binding interface for G protein and arrestin. Nat Commun 5:4801.

Takai Y, Sasaki T, and Matozaki T (2001) Small GTP-binding proteins. Physiol Rev 81:153-208.

Tan S, Li L, Chen T, Chen X, Tao L, Lin X, Tao J, Huang X, Jiang J, Liu H, et al. (2015) $\beta$-Arrestin-1 protects against endoplasmic reticulum stress/p53-upregulated modulator of apoptosis-mediated apoptosis via repressing p-p65/inducible nitric oxide synthase in portal hypertensive gastropathy. Free Radic Biol Med 87:69-83. Tarigopula M, Davis 3rd RT, Mungai PT, Ryba DM, Wieczorek DF, Cowan CL, Violin JD, Wolska BM, and Solaro RJ (2015) Cardiac myosin light chain phosphorylation and inotropic effects of a biased ligand, TRV120023, in a dilated cardiomyopathy model. Cardiovasc Res 107:226-234.

Thal DM, Homan KT, Chen J, Wu EK, Hinkle PM, Huang ZM, Chuprun JK, Song J, Gao E, Cheung JY, et al. (2012) Paroxetine is a direct inhibitor of G protein-coupled receptor kinase 2 and increases myocardial contractility. ACS Chem Biol 7 1830-1839.

Thomsen AR, Plouffe B, Cahill 3rd TJ, Shukla AK, Tarrasch JT, Dosey AM, Kahsai AW, Strachan RT, Pani B, Mahoney JP, et al. (2016) GPCR-G protein- $\beta$-arrestin super-complex mediates sustained G protein signaling. Cell 166:907-919.

Tobin AB, Butcher AJ, and Kong KC (2008) Location, location, location...site-specific GPCR phosphorylation offers a mechanism for cell-type-specific signalling. Trends Pharmacol Sci 29:413-420.

Tohoo A, Choy EW, Gesty-Palmer D, Pierce KL, Laporte S, Oakley RH, Caron MG, Lefkowitz RJ, and Luttrell LM (2003) The stability of the G protein-coupled receptor-beta-arrestin interaction determines the mechanism and functional consequence of ERK activation. J Biol Chem 278:6258-6267. 
Tohgo A, Pierce KL, Choy EW, Lefkowitz RJ, and Luttrell LM (2002) $\beta$-Arrestin scaffolding of the ERK cascade enhances cytosolic ERK activity but inhibits ERKmediated transcription following angiotensin AT1a receptor stimulation. $J$ Biol Chem 277:9429-9436.

Tomhave ED, Richardson RM, Didsbury JR, Menard L, Snyderman R, and Ali H (1994) Cross-desensitization of receptors for peptide chemoattractants: characterization of a new form of leukocyte regulation. J Immunol 153:3267-3275.

Vilardaga JP, Jean-Alphonse FG, and Gardella TJ (2014) Endosomal generation of cAMP in GPCR signaling. Nat Chem Biol 10:700-706.

Violin JD, DeWire SM, Yamashita D, Rominger DH, Nguyen L, Schiller K, Whalen EJ, Gowen M, and Lark MW (2010) Selectively engaging $\beta$-arrestins at the angiotensin II type 1 receptor reduces blood pressure and increases cardiac performance. J Pharmacol Exp Ther 335:572-579.

Vishnivetskiy SA, Hirsch JA, Velez MG, Gurevich YV, and Gurevich VV (2002) Transition of arrestin into the active receptor-binding state requires an extended interdomain hinge. J Biol Chem 277:43961-43967.

Vishnivetskiy SA, Hosey MM, Benovic JL, and Gurevich VV (2004) Mapping the arrestin-receptor interface: structural elements responsible for receptor specificity of arrestin proteins. J Biol Chem 279:1262-1268.

Vishnivetskiy SA, Gimenez LE, Francis DJ, Hanson SM, Hubbell WL, Klug CS, and Gurevich VV (2011) Few residues within an extensive binding interface drive receptor interaction and determine the specificity of arrestin proteins. J Biol Chem 286:24288-24299.

Vishnivetskiy SA, Schubert C, Climaco GC, Gurevich YV, Velez MG, and Gurevich VV (2000) An additional phosphate-binding element in arrestin molecule: implications for the mechanism of arrestin activation. J Biol Chem 275:41049-41057.

Walker JK and DeFea KA (2014) Role for $\beta$-arrestin in mediating paradoxical $\beta 2 \mathrm{AR}$ and PAR2 signaling in asthma. Curr Opin Pharmacol 16:142-147.

Walker JK, Fong AM, Lawson BL, Savov JD, Patel DD, Schwartz DA, and Lefkowitz RJ (2003) Beta-arrestin-2 regulates the development of allergic asthma. J Clin Invest 112:566-574.

Walters RW, Shukla AK, Kovacs JJ, Violin JD, DeWire SM, Lam CM, Chen JR, Muehlbauer MJ, Whalen EJ, and Lefkowitz RJ (2009) beta-Arrestin1 mediates nicotinic acid-induced flushing, but not its antilipolytic effect, in mice. $J$ Clin Invest 119:1312-1321.

Wang L, Martin B, Brenneman R, Luttrell LM, and Maudsley S (2009) Allosteric modulators of $\mathrm{G}$ protein-coupled receptors: future therapeutics for complex physiological disorders. J Pharmacol Exp Ther 331:340-348.

Wang P and DeFea KA (2006) Protease-activated receptor-2 simultaneously directs beta-arrestin-1-dependent inhibition and Galphaq-dependent activation of phosphatidylinositol 3-kinase. Biochemistry 45:9374-9385.

Wang P, Gao H, Ni Y, Wang B, Wu Y, Ji L, Qin L, Ma L, and Pei G (2003) Betaarrestin 2 functions as a G-protein-coupled receptor-activated regulator of oncoprotein Mdm2. J Biol Chem 278:6363-6370.

Wang P, Kumar P, Wang C, and Defea KA (2007) Differential regulation of class IA phosphoinositide 3 -kinase catalytic subunits p110 alpha and beta by proteaseactivated receptor 2 and beta-arrestins. Biochem $J$ 408:221-230.

Wang Y, Tang Y, Teng L, Wu Y, Zhao X, and Pei G (2006) Association of beta-arrestin and TRAF6 negatively regulates Toll-like receptor-interleukin 1 receptor signaling. Nat Immunol 7:139-147.

Whalen EJ, Rajagopal S, and Lefkowitz RJ (2011) Therapeutic potential of $\beta$-arrestin- and G protein-biased agonists. Trends Mol Med 17:126-139.

White KL, Robinson JE, Zhu H, DiBerto JF, Polepally PR, Zjawiony JK, Nichols DE, Malanga CJ, and Roth BL (2015) The G protein-biased k-opioid receptor agonist RB-64 is analgesic with a unique spectrum of activities in vivo. J Pharmacol Exp Ther 352:98-109.

White KL, Scopton AP, Rives ML, Bikbulatov RV, Polepally PR, Brown PJ, Kenakin T, Javitch JA, Zjawiony JK, and Roth BL (2014) Identification of novel functionally selective к-opioid receptor scaffolds. Mol Pharmacol 85:83-90.

Wilbanks AM, Fralish GB, Kirby ML, Barak LS, Li YX, and Caron MG (2004) Betaarrestin 2 regulates zebrafish development through the hedgehog signaling pathway. Science 306:2264-2267.

Witherow DS, Garrison TR, Miller WE, and Lefkowitz RJ (2004) beta-Arrestin inhibits NF-kappaB activity by means of its interaction with the NF-kappaB inhibitor IkappaBalpha. Proc Natl Acad Sci USA 101:8603-8607.

Woodall MC, Ciccarelli M, Woodall BP, and Koch WJ (2014) G protein-coupled receptor kinase 2: a link between myocardial contractile function and cardiac metabolism. Circ Res 114:1661-1670.

World C, Spindel ON, and Berk BC (2011) Thioredoxin-interacting protein mediates TRX1 translocation to the plasma membrane in response to tumor necrosis factor- $\alpha$ : a key mechanism for vascular endothelial growth factor receptor-2 transactivation by reactive oxygen species. Arterioscler Thromb Vasc Biol 31:1890-1897.

Wu N, Hanson SM, Francis DJ, Vishnivetskiy SA, Thibonnier M, Klug CS, Shoham M, and Gurevich VV (2006) Arrestin binding to calmodulin: a direct interaction between two ubiquitous signaling proteins. J Mol Biol 364:955-963.

Xiao K, McClatchy DB, Shukla AK, Zhao Y, Chen M, Shenoy SK, Yates 3rd JR, and Lefkowitz RJ (2007) Functional specialization of beta-arrestin interactions revealed by proteomic analysis. Proc Natl Acad Sci USA 104:12011-12016.

Xiao K, Sun J, Kim J, Rajagopal S, Zhai B, Villén J, Haas W, Kovacs JJ, Shukla AK, Hara MR, et al. (2010) Global phosphorylation analysis of beta-arrestin-mediated signaling downstream of a seven transmembrane receptor (7TMR). Proc Natl Acad Sci USA 107:15299-15304.
Xu TR, Baillie GS, Bhari N, Houslay TM, Pitt AM, Adams DR, Kolch W, Houslay MD, and Milligan G (2008) Mutations of beta-arrestin 2 that limit self-association also interfere with interactions with the beta2-adrenoceptor and the ERK1/2 MAPKs: implications for beta2-adrenoceptor signalling via the ERK1/2 MAPKs. Biochem $J$ 413:51-60.

Yang CH, Huang HW, Chen KH, Chen YS, Sheen-Chen SM, and Lin CR (2011) Antinociceptive potentiation and attenuation of tolerance by intrathecal $\beta$-arrestin 2 small interfering RNA in rats. Br $J$ Anaesth 107:774-781.

Yang X, Zhou G, Ren T, Li H, Zhang Y, Yin D, Qian H, and Li Q (2012) $\beta$-Arrestin prevents cell apoptosis through pro-apoptotic ERK1/2 and p38 MAPKs and antiapoptotic Akt pathways. Apoptosis 17:1019-1026.

Yoshihara E, Masaki S, Matsuo Y, Chen Z, Tian H, and Yodoi J (2014) Thioredoxin Txnip: redoxisome, as a redox switch for the pathogenesis of diseases. Front Immunol 4:514.

You HJ, How T, and Blobe GC (2009) The type III transforming growth factor-beta receptor negatively regulates nuclear factor kappa B signaling through its interaction with beta-arrestin2. Carcinogenesis 30:1281-1287.

Yu MC, Su LL, Zou L, Liu Y, Wu N, Kong L, Zhuang ZH, Sun L, Liu HP, Hu JH, et al. (2008) An essential function for beta-arrestin 2 in the inhibitory signaling of natural killer cells. Nat Immunol 9:898-907.

Yue R, Kang J, Zhao C, Hu W, Tang Y, Liu X, and Pei G (2009) Beta-arrestin1 regulates zebrafish hematopoiesis through binding to YY1 and relieving polycomb group repression. Cell 139:535-546.

Zhai P, Yamamoto M, Galeotti J, Liu J, Masurekar M, Thaisz J, Irie K, Holle E, Yu X, Kupershmidt S, et al. (2005) Cardiac-specific overexpression of AT1 receptor mutant lacking $\mathrm{G}$ alpha $\mathrm{q} / \mathrm{G}$ alpha i coupling causes hypertrophy and bradycardia in transgenic mice. J Clin Invest 115:3045-3056.

Zhan X, Gimenez LE, Gurevich VV, and Spiller BW (2011a) Crystal structure of arrestin-3 reveals the basis of the difference in receptor binding between two nonvisual subtypes. J Mol Biol 406:467-478.

Zhan X, Kaoud TS, Dalby KN, and Gurevich VV (2011b) Nonvisual arrestins function as simple scaffolds assembling the MKK4-JNK3 $\alpha 2$ signaling complex. Biochemistry 50:10520-10529.

Zhan X, Kaoud TS, Kook S, Dalby KN, and Gurevich VV (2013) JNK3 enzyme binding to arrestin-3 differentially affects the recruitment of upstream mitogenactivated protein (MAP) kinase kinases. J Biol Chem 288:28535-28547.

Zhan X, Perez A, Gimenez LE, Vishnivetskiy SA, and Gurevich VV (2014) Arrestin-3 binds the MAP kinase JNK3 $\alpha 2$ via multiple sites on both domains. Cell Signal 26: $766-776$.

Zhan X, Stoy H, Kaoud TS, Perry NA, Chen Q, Perez A, Els-Heindl S, Slagis JV, Iverson TM, Beck-Sickinger AG, et al. (2016) Peptide mini-scaffold facilitates JNK3 activation in cells. Sci Rep 6:21025.

Zhang TL, Fu JL, Geng Z, Yang JJ, and Sun XJ (2012) The neuroprotective effect of losartan through inhibiting AT1/ASK1/MKK4/JNK3 pathway following cerebral I/R in rat hippocampal CA1 region. CNS Neurosci Ther 18:981-987.

Zhang Y, Rodriguez AL, and Conn PJ (2005) Allosteric potentiators of metabotropic glutamate receptor subtype 5 have differential effects on different signaling pathways in cortical astrocytes. J Pharmacol Exp Ther 315:1212-1219.

Zhao M, Wimmer A, Trieu K, Discipio RG, and Schraufstatter IU (2004) Arrestin regulates MAPK activation and prevents NADPH oxidase-dependent death of cells expressing CXCR2. J Biol Chem 279:49259-49267.

Zhou B, Wang ZX, Zhao Y, Brautigan DL, and Zhang ZY (2002) The specificity of extracellular signal-regulated kinase 2 dephosphorylation by protein phosphatases. J Biol Chem 277:31818-31825.

Zhou L, Lovell KM, Frankowski KJ, Slauson SR, Phillips AM, Streicher JM, Stahl E, Schmid CL, Hodder P, Madoux F, et al. (2013) Development of functionally selective, small molecule agonists at kappa opioid receptors. J Biol Chem 288: 36703-36716.

Zhuang LN, Hu WX, Xin SM, Zhao J, and Pei G (2011) Beta-arrestin-1 protein represses adipogenesis and inflammatory responses through its interaction with peroxisome proliferator-activated receptor-gamma (PPARgamma). J Biol Chem 286:28403-28413.

Zhuang T, Vishnivetskiy SA, Gurevich VV, and Sanders CR (2010) Elucidation of inositol hexaphosphate and heparin interaction sites and conformational changes in arrestin-1 by solution nuclear magnetic resonance. Biochemistry 49: 10473-10485.

Zhuo Y, Vishnivetskiy SA, Zhan X, Gurevich VV, and Klug CS (2014) Identification of receptor binding-induced conformational changes in non-visual arrestins. J Biol Chem 289:20991-21002.

Zimmerman B, Beautrait A, Aguila B, Charles R, Escher E, Claing A, Bouvier M, and Laporte SA (2012) Differential $\beta$-arrestin-dependent conformational signaling and cellular responses revealed by angiotensin analogs. Sci Signal 5:ra33.

Zimmerman B, Simaan M, Lee M-H, Luttrell LM, and Laporte SA (2009) c-Srcmediated phosphorylation of AP-2 reveals a general mechanism for receptors internalizing through the clathrin pathway. Cell Signal 21:103-110.

Zoudilova M, Kumar P, Ge L, Wang P, Bokoch GM, and DeFea KA (2007) Betaarrestin-dependent regulation of the cofilin pathway downstream of proteaseactivated receptor-2. J Biol Chem 282:20634-20646.

Zoudilova M, Min J, Richards HL, Carter D, Huang T, and DeFea KA (2010) betaArrestins scaffold cofilin with chronophin to direct localized actin filament severing and membrane protrusions downstream of protease-activated receptor-2. J Biol Chem 285:14318-14329. 